\title{
Torsion Constraints in Supergeometry
}

\author{
John Lott* \\ I.H.E.S., F-91440 Bures-sur-Yvette, France \\ Received November 9, 1989; in revised form April 2, 1990
}

\begin{abstract}
We derive the torsion constraints for superspace versions of supergravity theories by means of the theory of $G$-structures. We also discuss superconformal geometry and superKähler geometry.
\end{abstract}

\section{Introduction}

Supersymmetry is a now well established topic in quantum field theory [WB, GGRS]. The basic idea is that one can construct actions in ordinary spacetime which involve both even commuting fields and odd anticommuting fields, with a symmetry which mixes the two types of fields. These actions can then be interpreted as arising from actions in a superspace with both even and odd coordinates, upon doing a partial integration over the odd coordinates. A mathematical framework to handle the differential topology of supermanifolds, manifolds with even and odd coordinates, was developed by Berezin, Kostant and others. A very readable account of this theory is given in the book of Manin [Ma].

The right notion of differential geometry for supermanifolds is less clear. Such a geometry is necessary in order to write supergravity theories in superspace. One could construct a supergeometry by $\mathbb{Z}_{2}$ grading what one usually does in (pseudo) Riemannian geometry, to have supermetrics, super Levi-Cività connections, etc. The local frame group which would take the place of the orthogonal group in standard geometry would be the orthosymplectic group. However, it turns out that this would be physically undesirable. Such a program would give more fields than one needs for a minimal supergravity theory, i.e. the fields would give a reducible representation of the superLorentz group. In order to get around this problem, the approach of Wess and Zumino [WZ] is to use the standard orthogonal group as the

* Permanent address as of September 1, 1990: Department of Mathematics, University of Michigan, Ann Arbor, MI 48109, USA 
structure group, but to choose selected components of the torsion tensor which can be nonzero. One then uses the Bianchi identities to find the consequences of the choice. If the choice is too stringent, one only finds flat geometries. If the choice is too lax, one gets too many fields in the supergravity theory. It is a well-developed technique to find the right torsion choices, but the geometric meaning is obscure and the method remains somewhat of an art.

To give an analogy, suppose that one has an almost complex manifold with a Hermitian structure. Let $\left\{e_{i}\right\}$ be a local unitary basis of the complexified tangent bundle. If one is told that a desirable set of torsion constraints is given by

$$
\begin{aligned}
& T_{i j k}=0, \\
& T_{i j \bar{k}}=T_{\bar{k} i j}-T_{\bar{k} j i},
\end{aligned}
$$

the geometric meaning of the constraints may not be clear. In fact, they are saying that the manifold is a Kähler manifold, which means that to first order around a point, the geometry of the manifold is the unitary geometry of $\mathbb{C}^{n}$.

We wish to give a similar interpretation of the torsion constraints of supergravity theory. Our approach will be to use Cartan's theory of $G$-structures [St, Gu, Kob]. The idea of this theory is as follows. Given a subgroup $G$ of the invertible endomorphisms of the tangent space and a reduction of the structure group of a manifold to $G$, one can ask whether the manifold is locally equivalent to a flat $G$-structure. Let us put a $G$-connection on the reduced frame bundle. Roughly speaking, the first-order flatness is measured by a combination of the components of the torsion tensor of the connection, which is constructed in such a way that the result is independent of the $G$-connection chosen. (For example, for (pseudo) Riemannian geometry this combination always vanishes, which gives Einstein's equivalence principle.) If one has first-order flatness, one can ask if there are higher order obstructions to flatness. These are given by the Spencer homology groups [Sp, Gu], which are an algebraic generalization of the Riemann curvature tensor and its covariant derivatives. By different choices of the group $G$, one obtains different geometries.

Our approach to supergeometry is to find the groups $G$ which give the torsion constraints of supergravity. We consider the geometries which come from these groups to be preferred, in that they do come from physics. We find that the appropriate groups have the following structure. If we locally decompose the tangent space into even and odd subspaces, then the group elements take the matrix form $\left(\begin{array}{cc}\varrho_{1}(A) & 0 \\ * & \varrho_{2}(A)\end{array}\right)$. Here $A$ is an element of the Spin group, $\varrho_{1}(A)$ is its representation as an orthogonal matrix, $\varrho_{2}$ is a spinor representation and $*$ lies in a Spin-invariant subspace $\mathscr{S}$ of endomorphisms from the even subspace to the odd subspace. (Different choices of $\mathscr{S}$ can give different geometries.) We will show explicitly that the torsion constraints for supergravity theories (at least those existing offshell) arise from the requirement of first-order flatness of such $G$ structures. Such structures have previously occurred in the work of Rosly and Schwarz in four dimensions [RS] and Giddings and Nelson in two dimensions [GN]. We also look at the geometric structures underlying superconformal geometry and superKähler geometry. 
The outline of this paper is as follows:

In Sect. II we collect the needed background on supermanifold theory.

In Sect. III we give a digression on the physical interpretation of supermanifold theory. There seems to be some confusion as to whether the sheaf-theoretic description of supermanifolds is adequate to describe both classical and quantum supersymmetric field theories. We show by the specific example of supergeodesic motion that it is, when properly interpreted. Although this material may be known to some, we have not seen it in the literature, and so we have included it.

In Sect. IV we define the model flat superspaces. We then define superRiemannian structure bundles and superRiemannian geometries in terms of $G$-structures. We work out the torsion conditions when the subspace $\mathscr{S}$ is the largest possible subspace.

In Sect. $\mathrm{V}$ we show by explicit calculation that with the right choice of the subspace $\mathscr{S}$, the torsion conditions of Sect. IV, along with the Bianchi identities, give the supergravity theories in 2, 3, 4, and 6 dimensions. Our purpose here is not to repeat known expressions for the torsion and curvature tensors, but to show how they follow from the general framework.

In Sect. VI we compute the Spencer homology groups when the subspace $\mathscr{S}$ is the largest possible.

Conformal structures have a greater role in supergeometry than in ordinary geometry. Conformal supergravity theories can be used as a technical tool to construct Lorentzian supergravity theories [GGRS]. Conformal groups also arise when one looks at the diffeomorphisms of the flat space which preserve the flat space torsion tensor and the odd subspace of the tangent space. One finds that the corresponding Lie algebra is a subalgebra $g^{(-1)} \oplus g^{(-1 / 2)} \oplus g^{(0)}$ of the superconformal algebra. This gives a strong analogy between superconformal geometry and the pseudoconformal geometry of CR manifolds, in which the flat-space torsion tensor is given by the Levi form of the sphere. In Sect. VII we find the curvature obstructions to superconformal flatness, in analogy to the work of Chern-Moser on CR geometry [CM].

Although the local geometry of supermanifolds of one complex dimension is well understood, it is not a priori clear how to generalize this to more complex dimensions. In Sect. VIII we look at one approach, which is to extend Kähler geometry. This can be done following the above approach to superRiemannian geometry, and using the fact that the spinors have a natural description in terms of $(p, 0)$ forms on a Kähler manifold. We define three types of such structures, which we call superKähler, $\mathbb{C}^{*}$-extended superKähler and weak superKähler structures. We show that in one complex dimension, superKähler structures are always flat, and both $\mathbb{C}^{*}$-extended superKähler and weak superKähler structures are equivalent to the superRiemannian geometry. In two complex dimensions we show that the reduced manifold for a superKähler or $\mathbb{C}^{*}$-extended superKähler structure is always a locally Hermitian symmetric space (although we do not claim that any such symmetric space is the reduced manifold for such a structure). We show that a weak superKähler structure is equivalent to an superRiemannian structure with an $\mathbb{H}^{*}$ internal symmetry group, for which the strictly even part of the curvature form is $u(2)$ valued. However, our discussion here is incomplete.

In Sect. IX and X we briefly discuss two related topics. In Sect. IX we discuss the constraints for supergauge theory, and give an example in Riemannian 4-space. In 
Sect. X we discuss when the torsion constraints for superconformal geometry can be seen as arising from integrability conditions for the local embedding of the supermanifold in a flat complex or quaternionic superspace, as in the work of Ogievetsky-Sokatchev [OS] and Rosly-Schwarz [RS] for four-dimensional supergravity.

Notation. We will follow the notation of [WB], in which letters from the beginning of the alphabet denote frame indices, letters from the middle of the alphabet denote coordinate indices, small Latin letters denote even indices, small Greek letters denote odd indices and capital letters denote even or odd indices.

I wish to thank Dan Burns for helpful explanations of CR geometry, and Ofer Gabber for a helpful conversation. I thank M. Berger and the IHES for their hospitality while part of this research was performed.

Note. Spencer homology groups have been previously computed in a supersymmetric case in [RS2]. I thank the referee for bringing this reference to my attention.

\section{Review of Supermanifold Theory}

We will assume a knowledge of superalgebra, as given in [Le] or [Ma]. The idea underlying supermanifold theory is that one can do much of ordinary topology and geometry by working with the ring of functions on a manifold, instead of the points of the manifold. A supermanifold is defined by its "ring of functions," which is now generalized to be a supercommutative ring. Some conditions are put on this idea to make it workable. Let us recall the definition of a supermanifold [Ma]. (The definition is the same whether one is working in the smooth, analytic or complex analytic categories, provided that one makes the obvious changes.) A supermanifold $X$ consits of a pair $\left(M, \mathcal{O}_{M}\right)$ such that $M$ is a smooth manifold, $\mathcal{O}_{M}$ is a sheaf of supercommutative rings over $M$ and certain conditions are satisfied. To give the conditions $(*)$, we will need the following notation:

Definition 1. Let $\mathcal{O}_{M, 1}$ denote the sheaf of vector spaces formed by the odd part of $\mathcal{O}_{M}$. Let $\mathscr{J}_{M}$ denote the sheaf $\mathcal{O}_{M, 1}+\mathcal{O}_{M, 1}^{2}$ of ideals of nilpotent elements. Then the conditions $(*)$ are

1. $\mathcal{O}_{M} / \mathscr{J}_{M}$ is the structure sheaf of $M$.

2. $\mathscr{J}_{M} / \mathscr{J}_{M}^{2}$ is a locally free sheaf of $\mathcal{O}_{M} / \mathscr{J}_{M}$ modules.

3. $\mathcal{O}_{M}$ is locally isomorphic to the sheaf $\Lambda_{\mathscr{O}_{M} / \mathscr{I}_{M}}^{*}\left(\mathscr{J}_{M} / \mathscr{J}_{M}^{2}\right)$ of exterior algebras.

We will call $\mathcal{O}_{M}$ the structure sheaf for $X,\left(M, \mathcal{O}_{M} / \mathscr{J}_{M}\right)$ the reduced manifold of $X$, and the sections of $\mathcal{O}_{M}$ will be called the functions on $X$.

The standard example of a supermanifold is constructed from a smooth vector bundle $E$ over $M$. Define the sheaf $\mathcal{O}_{M}$ by saying that over an open set $U \subset M, \mathcal{O}_{U}$ is the ring of smooth sections of the Grassmannian bundle $\left.\Lambda^{*} E\right|_{U}$. One can show that any smooth supermanifold arises from such a construction, although not canonically [Ga, Ba]. We will be concerned with smooth supermanifolds in this paper. For a trivial $\mathbb{R}^{q}$ bundle over $\mathbb{R}^{p}$, we will denote the corresponding supermanifold by $\mathbb{R}^{p \mid q}$.

A supermanifold has both even and odd local coordinates in the following sense. Let $p$ be the dimension of $M$ and let $q$ be the dimension of the locally free sheaf 
$\mathscr{J}_{M} / \mathscr{J}_{M}^{2}$. Then for any point $n \in M$, we can find a neighborhood $U$ of $n$ with local coordinates $\left\{x^{m}\right\}_{m=1}^{p}$ and sections $\left\{\eta^{\mu}\right\}_{\mu=1}^{q}$ of $\mathcal{O}_{U}$ such that any function $\left.f \in \mathcal{O}\right|_{U}$ can be written as $\sum f_{I}(x) \eta^{I}$, where $I$ is an increasing multi-index from $\{1,2, \ldots, q\}$ and each $f_{I}(x)$ is in $C^{\infty}(U)$.

Much of the theory of differentiable manifolds goes over to the case of supermanifolds without trouble [Ma]. In order to fix notation, we will recall some of this. Let $\Pi \mathcal{O}_{M}$ denote the sheaf $\mathcal{O}_{M}$ with the parities reversed. A locally free sheaf of rank $r \mid s$ is a sheaf of graded $\mathcal{O}_{M}$ modules which is locally isomorphic to $\mathcal{O}_{M}^{r \mid s}=\mathcal{O}_{M}^{r} \oplus\left(\Pi \mathcal{O}_{M}\right)^{s}$. The sheaf of tangent vectors $T X$ is the sheaf of local derivations of $\mathcal{O}_{M}$. It has rank $p \mid q$, and has a basis of local sections consisting of the even derivatives $\left\{\partial_{x^{m}}\right\}_{m=1}^{p}$ and the odd derivatives $\left\{\partial_{\eta^{\mu}}\right\}_{\mu=1}^{q}$. The sheaf of cotangent vectors $T^{*} X$ is the dual of $T X$, and has rank $p \mid q$ and local sections $\left\{d x^{m}\right\}_{m=1}^{p}$ and $\left\{d \eta^{\mu}\right\}_{\mu=1}^{q}$. The sheaf of differential forms $\Lambda^{*} X$ is the exterior algebra of $T^{*} X$, and has the local relations

$$
\begin{aligned}
& d x^{m} \wedge d x^{n}=-d x^{n} \wedge d x^{m} \\
& d x^{m} \wedge d \eta^{\mu}=-d \eta^{\mu} \wedge d x^{m} \\
& d \eta^{\mu} \wedge d \eta^{v}=d \eta^{v} \wedge d \eta^{\mu}
\end{aligned}
$$

$\Lambda^{*} X$ has an even exterior derivative $d$. To follow the notation of [WB], we will let $d$ act from the right, so that

$$
d(\omega \wedge \sigma)=\omega \wedge d \sigma+(-1)^{\operatorname{deg}(\sigma)} d \omega \wedge \sigma
$$

where $\operatorname{deg}(\sigma)$ is the degree of $\sigma$ as a differential form, and for $f \in C^{\infty}(X)$, we have locally

$$
d f=\sum_{M} d z^{M} \partial_{M} f
$$

Let us note that there are some differences between integration on supermanifolds and integration of exterior forms on ordinary manifolds. On a supermanifold, one integrates sections of a rank one sheaf called the Berezinian (see [Ma]).

A morphism $\Phi$ between a supermanifold $X$ and a supermanifold $X^{\prime}$ is a pair $(\phi, \psi)$, where $\phi$ is a smooth mapping from $M$ to $M^{\prime}$ and $\psi: \mathcal{O}_{M^{\prime}} \rightarrow \phi_{*}\left(\mathcal{O}_{M}\right)$ is an even morphism of sheafs of rings which is local with respect to $\phi$. If $(x, \eta)$ are local coordinates around a point $n \in M$ and $\left(x^{\prime}, \eta^{\prime}\right)$ are local coordinates around $\phi(n)$ then $\Phi$ is locally given by

$$
\Phi^{*}\left(x^{\prime m}\right)=\sum_{I \text { even }} f_{I}^{m}(x) \eta^{I} \text { and } \Phi^{*}\left(\eta^{\prime \mu}\right)=\sum_{I \text { odd }} g_{I}^{\mu}(x) \eta^{I}
$$

for smooth functions $\left\{f_{I}^{m}(x)\right\}$ and $\left\{g_{I}^{\mu}(x)\right\}$ (where $I$ is a multi-index) with $f_{\emptyset}^{m}(x)=\phi^{*}\left(x^{\prime m}\right)$.

One point of supermanifold theory which may not be familiar is the notion of a super Lie group. Recall [Ka] that a super Lie algebra $g$ is given by an ordinary Lie algebra (over $\mathbb{R}$ or $\mathbb{C}$ ) $g_{0}$ and a finite dimensional vector space $g_{1}$ such that

1. There is a representation $\varrho$ of $g_{0}$ on $g_{1}$.

2. There is a $g_{0}$-equivariant symmetric map $d: S^{2}\left(g_{1}\right) \rightarrow g_{0}$.

3. $\forall \eta_{1}, \eta_{2}$ and $\eta_{3} \in g_{1}, \varrho\left(d\left(\eta_{1}, \eta_{2}\right)\right) \eta_{3}+\varrho\left(d\left(\eta_{2}, \eta_{3}\right)\right) \eta_{1}+\varrho\left(d\left(\eta_{3}, \eta_{1}\right)\right) \eta_{2}=0$. 
The commutation relations are given by

$1^{\prime} .[X, Y]_{g}=[X, Y]_{g 0}$ for $X, Y \in g_{0}$.

$2^{\prime} .[X, Y]_{g}=\varrho(x) Y$ for $X \in g_{0}, Y \in g_{1}$.

3'. $[X, Y]_{g}=d(X, Y)$ for $X, Y \in g_{1}$.

One can define a super Lie group to be a supermanifold for which the space of "distributions of finite support" has a graded Hopf algebra structure with antipode [Kos]. Let us note that with this definition, what is sometimes called the general linear group of a graded vector space $W$, namely the invertible endomorphisms Aut $(W)$, is not a super Lie group, but instead gives coordinates for the super Lie group $G L(W)$. One way to obtain super Lie groups is as follows.

Proposition 1. Let $G$ be a Lie group and let $\varrho$ be a representation of $G$ on a finite dimensional vector space $V$. Suppose that there is a G-equivariant map $d: S^{2} V \rightarrow g$ which satisfies Condition 3 above. Let $E$ be the trivial vector bundle $G \times V$ and let $X$ be the associated supermanifold, with reduced space $G$. Then $X$ has a super Lie group structure.

Proof. See [Kos].

\section{Relation of Supermanifold Theory to Physics}

There seems to be some confusion in the literature as to whether the above formalism can handle the supersymmetric theories of theoretical physics (see for example the discussion in [DS]). Let us give a simple example. The formal Lagrangian for an $N=\frac{1}{2}$ supersymmetric theory of maps from $\mathbb{R}^{1 \mid 1}$ to $\mathbb{R}$ is

$$
L=\frac{1}{2} \int_{\mathbb{R}^{1}}\left(x^{\prime 2}+i \psi \psi^{\prime}\right) d T,
$$

where $x$ is an even function and $\psi$ is an odd function. One might wish to interpret $x$ and $\psi$ as functions on $\mathbb{R}^{1 \mid 1}$, in which case

$$
x \equiv f(T) \quad \text { and } \quad \psi \equiv g(T) \eta
$$

for functions $f, g \in C^{\infty}\left(\mathbb{R}^{1}\right)$. An immediate problem is that $\psi \psi^{\prime}$ would then be identically zero.

There is a natural resolution of this problem. Note that the space Mor $\left(X, X^{\prime}\right)$ of morphisms between two supermanifolds $X$ and $X^{\prime}$ is a space, not a superspace. We want a superspace of maps from $\mathbb{R}^{1 \mid 1}$ to $\mathbb{R}$. More generally, in analogy with the ordinary case, let us define a superspace $\operatorname{Map}\left(X, X^{\prime}\right)$ of maps between supermanifolds $X$ and $X^{\prime}$ by requiring that

$$
\operatorname{Mor}\left(Z, \operatorname{Map}\left(X, X^{\prime}\right)\right)=\operatorname{Mor}\left(Z \times X, X^{\prime}\right)
$$

for all supermanifolds $Z$. In the case $X=\mathbb{R}^{1 \mid 1}$ and $X^{\prime}=M^{\prime}$, an ordinary manifold, one finds by taking $Z=\mathbb{R}^{0 \mid 0}$ that the base space of $\operatorname{Map}\left(\mathbb{R}^{1 \mid 1}, M^{\prime}\right)$ is the infinitedimensional ordinary space $\operatorname{Map}\left(\mathbb{R}^{1}, M^{\prime}\right)$. By taking $Z=\mathbb{R}^{0 \mid 1}$, one finds that if $\operatorname{Map}\left(\mathbb{R}^{1 \mid 1}, M^{\prime}\right)$ comes from a vector bundle $E$ over Map $\left(\mathbb{R}^{1}, M^{\prime}\right)$ then $E$ must be the (smooth) cotangent bundle of $\operatorname{Map}\left(\mathbb{R}^{1}, M^{\prime}\right)$, as defined in $[\mathrm{Pa}]$. In fact, $\operatorname{Map}\left(\mathbb{R}^{1 \mid 1}, M^{\prime}\right)$ is the superspace whose functions are differential forms on $\operatorname{Map}\left(\mathbb{R}^{1}, M^{\prime}\right)[$ Lo]. 
Given an ordinary Riemannian metric $\langle$,$\rangle on M^{\prime}$, the Lagrangian

$$
L=\frac{1}{2} \int_{\mathbb{R}^{1}}\left(\left\langle x^{\prime}, x^{\prime}\right\rangle+i\left\langle\psi, \nabla_{x^{\prime}} \psi\right\rangle\right) d T
$$

is actually a function on $\operatorname{Map}\left(\mathbb{R}^{1 \mid 1}, M^{\prime}\right)$, namely the sum of a zero-form and a twoform on $\operatorname{Map}\left(\mathbb{R}^{1}, M^{\prime}\right)$ [At]. The quantum theory with Lagrangian $L$ can be constructed by considering the vacuum expectation as a certain linear functional acting on functions on $\operatorname{Map}\left(\mathbb{R}^{1 \mid 1}, M^{\prime}\right)$ [Lo].

One can consider the classical theory with Lagrangian $L$ either from the Hamiltonian viewpoint or the Lagrangian viewpoint. The Hamiltonian approach is to consider the supermanifold $Y$ with reduced space $T^{*} M^{\prime}$ which is constructed from the vector bundle $E=\pi^{*} T^{*} M^{\prime}$, where $\pi: T^{*} M^{\prime} \rightarrow M^{\prime}$ is the projection map. Let $\left\{x^{m}\right\}$ be local coordinates for $M^{\prime}$, let $\left\{e_{a}\right\}$ be a local orthonormal frame and let $\left\{\tau^{a}\right\}$ be the dual coframe. Then there are local coordinates on $T^{*} M^{\prime}$ given by

$$
(m, p) \rightarrow\left\{x^{m}(m),\left\langle p, e_{a}\right\rangle\right\} .
$$

There are local sections $\left\{\eta^{\alpha}\right\}$ of $\pi^{*} T^{*} M^{\prime}$ given by $(m, p) \rightarrow\left(m, p, \tau^{\alpha}\right)$ and so we have coordinates $\left\{x^{m}, p_{a}, \eta^{\alpha}\right\}$ for $Y$. It is convenient to define a new coordinate $\pi_{a}$ by

$$
\pi_{a}=p_{a}-\frac{1}{2} i \Gamma_{\alpha \beta a} \eta^{\alpha} \eta^{\beta}
$$

Consider the local basis of $T^{*} Y$ given by

$$
\left\{\tau^{a}, D \pi_{a} \equiv d \pi_{a}-\Gamma_{a j}^{k} \pi_{k} \tau^{j} \text { and } D \eta^{\alpha} \equiv d \eta^{\alpha}+\Gamma_{\beta a}^{\alpha} \tau^{a} \eta^{\beta}\right\}
$$

Proposition 2. The two-form

$$
\omega=D \pi^{a} \wedge \tau^{a}+\frac{1}{2} i D \eta^{\alpha} D \eta^{\alpha}+\frac{1}{4} R_{\alpha \beta a b} \eta^{\alpha} \eta^{\beta} \tau^{a} \wedge \tau^{b}
$$

is supersymplectic.

We omit the proof.

From $\omega$ we derive a superPoisson algebra $\{\circ, \circ\}$. There are functions $Q=-i \eta^{a} \pi_{a}$, the supercharge, and $H=\frac{1}{2}\left(\pi^{a}\right)^{2}$, the Hamiltonian, with $\{Q, Q\}=2 i H$. The time evolution of a function on $Y$ is given by $d f / d t=-\{H, f\}$.

From the Lagrangian viewpoint, the equations of motion derived from $L$ are

$$
A^{m}(T) \equiv\left(x^{m}\right)^{\prime \prime}+\Gamma_{n p}^{m}\left(x^{n}\right)^{\prime}\left(x^{p}\right)^{\prime}-\frac{1}{2} i R_{n \alpha \beta}^{m}\left(x^{n}\right)^{\prime} \eta^{\alpha} \eta^{\beta}=0
$$

and

$$
B^{\alpha}(T) \equiv\left(\dot{\eta}^{\alpha}\right)^{\prime}+\Gamma_{\beta m}^{\alpha}\left(x^{m}\right)^{\prime} \eta^{\beta}=0
$$

In order to form the superspace $\mathscr{C}$ of classical solutions, it is necessary to form the formal quotient of the space of functions on $\operatorname{Map}\left(\mathbb{R}^{1 / 1}, M^{\prime}\right)$ by the ideal generated by $\left\{A^{m}(T), B^{\alpha}(T)\right\}_{m, \alpha, T}$. In order to see the equivalence with the Hamiltonian approach, recall that in ordinary classical mechanics one can identify the space of solutions of the equations of motion with the phase space, by evaluating the solutions at a fixed time. There is a symplectic form on the space of solutions which becomes identified with the symplectic form on the phase space [Se, Sz, CW]. In our case one can convince oneself that given a fixed $T, \mathscr{C}$ has the local coordinates $x^{m}(T)$, $\left(x^{m}\right)^{\prime}(T)$, and $\eta^{\alpha}(T)$, which gives the identification with the superphase space of the Hamiltonian approach. Choosing a different value $T^{\prime}$ of the time corresponds to a 
different choice of local coordinates of $\mathscr{C}$, which is related to the original choice by the time evolution operator $\exp \left(-\left(T^{\prime}-T\right)\{H, \circ\}\right)$.

We will be concerned in this paper with superspaces of geometrical structures on a supermanifold. The idea is to define these superspaces as universal objects with respect to some pullback property. (For a definition of superTeichmüller space in this vein, see [LR].) For example, in the ordinary case, one could define the space $\operatorname{Met}(M)$ of Riemannian metrics on $M$ as a space such that for any manifold $Z$, the space $\operatorname{Map}(Z, \operatorname{Met}(M))$ is the space of vertical metrics on the fibered space $M \rightarrow M \times Z$. Similarly, one can define the space $\operatorname{Met}(M) / \operatorname{Diff}(M)$ as a space such<smiles>[3H][CH]</smiles>

that for any manifold $Z$, the $\operatorname{space} \operatorname{Map}(Z, \operatorname{Met}(M) / \operatorname{Diff}(M))$ is the space of fiber spaces $M \rightarrow P$ with vertical Riemannian geometries, i.e. an element of $\downarrow$

$\operatorname{Met}(M) / \operatorname{Diff}(M)$ on each fiber.

In the super case, given a notion of a space of geometries on a supermanifold $X$, we will define a superspace $\operatorname{Geom}(X)$ by requiring that for all supermanifolds $Z$, $\operatorname{Mor}(Z, \operatorname{Geom}(X))$ is the space of vertical geometries on the fibered supermanifold $X \rightarrow X \times Z$.

Let $Z_{\text {red }}$ denote the base space of $Z$ and let $U$ be an open set in $Z_{\text {red }}$. Then the ring of functions of the pre-image of $U$ in $(X \times Z)_{\text {red }}$ will be $\mathcal{O}_{X} \otimes \mathcal{O}_{U}$. That is, to do local calculations we can deal with functions on $X$ which take value in the superalgebra $B=\mathcal{O}_{U}$. If $\{x, \eta\}$ are local coordinates for $X$ then the even functions have the form $f=\sum_{I} f_{I}(x) \eta^{I}$, where $f_{I}(x)$ is an (even or odd) element of $B$ if $I$ is an (even or odd) multi-index. In what follows, we will omit explicit mention of the algebra $B$, but all local calculations are to be understood in this way.

\section{SuperRiemannian Structures}

Let us define the super Euclidean group. Let $\chi$ be the nondegenerate quadratic form on $\mathbb{R}^{p}=\mathbb{R}^{\left(p_{+}\right)} \oplus \mathbb{R}^{\left(p_{-}\right)}$given by

$$
\left(v \oplus w, v^{\prime} \oplus w^{\prime}\right)=\left\langle v, v^{\prime}\right\rangle-\left\langle w, w^{\prime}\right\rangle .
$$

For simplicity of notation, we will let $S O\left(p_{+}, p_{-}\right)$denote the connected component of the identity of the corresponding special orthogonal group. Let $\pi$ denote the standard representation of $S O\left(p_{+}, p_{-}\right)$on $\mathbb{R}^{p}$. Let $\operatorname{Spin}\left(p_{+}, p_{-}\right)$denote the spin group, a double cover of $S O\left(p_{+}, p_{-}\right)$. Let $V$ be a real vector space of dimension $q$ on which $\operatorname{Spin}\left(p_{+}, p_{-}\right)$has a faithful spinor representation $\varrho$. That is, there are matrices $\left\{\gamma_{a}\right\}_{a=1}^{p} \in$ End $(V)$ which satisfy

$$
\gamma_{a} \gamma_{b}+\gamma_{b} \gamma_{a}=2 \chi_{a b},\left(\gamma_{a}\right)^{\dagger}=\chi_{a a} \gamma_{a}
$$


Put $\sigma_{a b}=\left[\gamma_{a}, \gamma_{b}\right] / 4$. Suppose that there is an invertible matrix $C \in \operatorname{End}(V)$ which satisfies

$$
C \gamma_{a} C^{-1}=\alpha \gamma_{a}{ }^{T}, C^{T}=\alpha C
$$

with $\alpha= \pm 1$. Then there is an $\operatorname{Spin}\left(p_{+}, p_{-}\right)$invariant charge conjugation operator on $V$ given by $\psi \rightarrow \psi^{c}=C^{-1} \psi$.

Definition 2. The super Euclidean algebra $e$ on $\mathbb{R}^{p} \mid V$ is the super Lie algebra with even part $s o\left(p_{+}, p_{-}\right) \oplus \mathbb{R}^{p}$ and odd part $V$, with commutation relations

$$
\begin{aligned}
& {\left[M \oplus P, M^{\prime} \oplus P^{\prime}\right]=\left[M, M^{\prime}\right] \oplus\left(\pi(M) P^{\prime}-\pi\left(M^{\prime}\right) P\right) \oplus 0,} \\
& {[M \oplus P, Q]=0 \oplus 0 \oplus \varrho(M) Q,} \\
& {\left[Q, Q^{\prime}\right]=0 \oplus Q^{T}\left(\gamma C^{-1}\right) Q^{\prime} \oplus 0 .}
\end{aligned}
$$

In terms of components,

$$
\begin{aligned}
& {\left[M_{\mu \nu}, M_{\sigma \tau}\right]=\eta_{v \sigma} M_{\mu \tau}-\eta_{\mu \sigma} M_{v \tau}-\eta_{v \tau} M_{\mu \sigma}+\eta_{\mu \tau} M_{v \sigma},} \\
& {\left[M_{\mu \nu}, P_{\sigma}\right]=\eta_{\nu \sigma} P_{\mu}-\eta_{\mu \sigma} P_{\nu},} \\
& {\left[M_{\mu \nu}, Q_{a}\right]=\left(\sigma_{\mu \nu}\right)_{a}^{b} Q_{b},} \\
& {\left[P_{\mu}, P_{v}\right]=\left[P_{\mu}, Q_{a}\right]=0,} \\
& {\left[Q_{a}, Q_{b}\right]=\eta^{\mu \nu}\left(\gamma_{\mu} C^{-1}\right)_{a b} P_{v} .}
\end{aligned}
$$

The super Euclidean group $\mathscr{E}$ on $\mathbb{R}^{p} \mid V$ is the corresponding super Lie group, as defined in Proposition 1, with reduced manifold $\operatorname{Spin}\left(p_{+}, p_{-}\right) \tilde{\times} \mathbb{R}^{p}$. This acts on $\mathbb{R}^{p} \mid V$, and the corresponding representation of $e$ by vector fields on $\mathbb{R}^{p} \mid V$ is given by

$$
\begin{aligned}
& P_{\mu}(x, \eta)=\partial_{\mu}, \\
& M_{\mu \nu}(x, \eta)=x_{v} \partial_{\mu}-x_{\mu} \partial_{\nu}, \\
& Q_{a}(x, \eta)=\partial_{a}+\frac{1}{2}\left(\gamma^{\mu} C^{-1}\right)_{a}^{\cdot b} \eta_{b} \partial_{\mu} .
\end{aligned}
$$

We can define a model geometry on $\mathbb{R}^{p} \mid V$ with $\mathscr{E}$ as an automorphism group by writing $\mathbb{R}^{p} \mid V$ as $\mathscr{E} / \operatorname{Spin}\left(p_{+}, p_{-}\right)$and using the decomposition

$$
e=\operatorname{spin}\left(p_{+}, p_{-}\right) \oplus\left(\mathbb{R}^{p} \oplus V\right)
$$

to put a canonical connection on $\mathbb{R}^{p} \mid V[\mathrm{KN}]$. To make this more explicit, let $s$ be a global section of the frame bundle of $\mathbb{R}^{p} \mid V$ given by

$$
s(x, \eta)=\left(D_{a}, D_{\alpha}\right) \equiv\left(\partial_{a}, \partial_{\alpha}-\frac{1}{2}\left(\gamma^{a} C^{-1}\right)_{\alpha}^{\beta} \eta_{\beta} \partial_{a}\right)
$$

and let $P_{0}$ be the Spin $\left(p_{+}, p_{-}\right)$subbundle of the frame bundle which includes the section $s$. Let $\pi$ denote the projection map from $P_{0}$ to $\mathbb{R}^{p} \mid V$. Let $\omega$ be the $\operatorname{Spin}\left(p_{+}, p_{-}\right)$connection on $P_{0}$ which vanishes when pulled back to $\mathbb{R}^{p} \mid V$ by $s$. Then one can verify that $\mathscr{E}$ acts by automorphisms on $P_{0}$ and preserves the connection $\omega$.

In order to discuss when another $\operatorname{Spin}\left(p_{+}, p_{-}\right)$structure is approximated by the model geometry, let us recall the notion of a torsion tensor for a $G$-structure [St]. Let $M$ be a manifold whose tangent space at a point is isomorphic to a vector space $W$ and let $P$ be a reduction of the frame bundle of $M$ to a group $G$. Let $\tau$ denote the 
canonical form of $P$, an $W$-valued horizontal 1 -form on $P$. Given a connection $\omega$ on $P$, the torsion $T$ is an $W$-valued horizontal 2 -form on $P$ defined by

$$
T^{A}=d \tau^{A}+\tau^{B} \wedge \omega_{B}{ }^{A} .
$$

Let $\omega^{\prime}=\omega+\Delta \omega$ be another $G$-connection on $P$, where $\Delta \omega$ is a $g$-valued equivariant horizontal 1 -form on $P$. Then

$$
T^{A}-T^{A}=\tau^{B} \wedge(\Delta \omega)_{B}{ }^{A} \equiv \tau^{B} \wedge \tau^{C}(\Delta \omega)_{C B}{ }^{A} .
$$

Let $\delta: W^{*} \otimes g \rightarrow \operatorname{Hom}(W \wedge W, W)$ be defined by

$$
\delta\left(w^{*}, X\right)\left(w^{\prime}, w^{\prime \prime}\right)=w^{*}\left(w^{\prime \prime}\right) X\left(w^{\prime}\right)-w^{*}\left(w^{\prime}\right) X\left(w^{\prime \prime}\right) .
$$

Let $g^{(1)}$ denote $\operatorname{Ker} \delta$. Let $H^{0,2}$ denote $\operatorname{Hom}(W \wedge W, W) / \operatorname{Im} \delta$. Then to each point $p \in P$ there is associated a class $C(p) \in H^{0,2}$, defined independently of $\omega$. There is an action of $G$ on $H^{0,2}$, and if $p g^{-1}$ denotes a point in the same fiber of $P$ as $p$ then $C\left(p g^{-1}\right)$ differs from $C(p)$ by a $g$ action.

Now suppose that there is a diffeomorphism $\phi: M \rightarrow \mathbb{R}^{m} \mid V$ for which the pushforward of the frame bundle induces a diffeomorphism $\phi_{*}: P \rightarrow P_{0}$. Then it follows that for any point $p \in P, C(p)$ must equal $C(\phi(p))$, which lies in the $G$-orbit of $C(s(\pi(\phi(p))))$. Thus the $G$-orbit of $C(\phi(p))$ gives an obstruction to the first-order flatness of $M$. If $C(p)$ equals $C(\phi(p))$ then we can choose a connection form $\omega$ at $p$ so that the torsion of $\omega$ at $p$ equals the torsion of the model space at $\phi(p)$. There is a freedom of $g^{(1)}$ in determining $\omega$.

In the supersymmetric case, if we write the torsion tensor in the form

$$
T^{A}=\frac{1}{2} \tau^{C} \wedge \tau^{B} T_{B C}{ }^{A}
$$

then one finds that in the model geometry, along the section $s$, the only nonvanishing components of the torsion tensor $T_{0}$ are

$$
\left(T_{0}\right)_{\alpha \beta}^{a}=\left(\gamma^{a} C^{-1}\right)_{\alpha \beta} .
$$

In order to see the consequences of first-order flatness, let us first look at the case of the smallest possible structure group consistent with a Riemannian structure on the reduced space, mainly $G=\operatorname{Spin}\left(p_{+}, p_{-}\right)$.

Proposition 3. For $\operatorname{Spin}\left(p_{+}, p_{-}\right)$acting on $W=\mathbb{R}^{p} \mid V, g^{(1)}=0$. A tensor $T \in \operatorname{Hom}(W \wedge W, W)$ lies in the same orbit of $H^{0,2}$ as $T_{0}$ if and only if

$$
\begin{aligned}
& T_{a b}{ }^{\gamma}=0, \\
& T_{\alpha \beta}{ }^{c}=\left(\gamma^{c} C^{-1}\right)_{\alpha \beta}, \\
& T_{a \beta}{ }^{\gamma}=\frac{1}{4}\left(T_{a b c}-T_{a c b}-T_{b c a}\right)\left(\sigma^{c b}\right)_{\beta}{ }^{\gamma}, \\
& T_{\alpha \beta}{ }^{\gamma}=\frac{1}{2} T_{\alpha a c}\left(\sigma^{c a}\right)_{\beta}{ }^{\gamma}+\frac{1}{2} T_{\beta a c}\left(\sigma^{c a}\right)_{\alpha}{ }^{\gamma} .
\end{aligned}
$$

Proof. Let $M$ denote an element of $W^{*} \otimes g$. whose image in $W^{*} \otimes\left(W^{*} \otimes W\right)$ we will write as $\left(M_{A}\right)_{B}{ }^{C}$. If $S$ denotes the image of $M$ in $\operatorname{Hom}(W \wedge W, W)$ then the 
components of $S$ are

$$
\begin{aligned}
& S_{a b}{ }^{c}=\left(M_{a}\right)_{b}{ }^{c}-\left(M_{b}\right)_{a}^{c}, \\
& S_{a b}{ }^{\gamma}=0, \\
& S_{a \beta}{ }^{c}=-\left(M_{\beta}\right)_{a}^{c}, \\
& S_{a \beta}{ }^{\gamma}=\left(M_{a}\right)_{\beta}^{\gamma}, \\
& S_{\alpha \beta}{ }^{c}=0, \\
& S_{\alpha \beta}^{\gamma}=\left(M_{\alpha}\right)_{\beta}^{\gamma}+\left(M_{\beta}\right)_{\alpha}^{\gamma} .
\end{aligned}
$$

Because the representation $\varrho$ is assumed to be faithful, it follows from (45) and (46) that $g^{(1)}=0$. From $(43),\left(M_{a}\right)_{b}{ }^{c}$ can be written in terms of $S_{a b}{ }^{c}$. Using the fact that

and

$$
\left(M_{a}\right)_{\beta}^{\gamma}=\frac{1}{2}\left(M_{a}\right)_{b}^{c}\left(\sigma_{c}^{b}\right)_{\beta}^{\gamma}
$$

$$
\left(M_{\alpha}\right)_{\beta}^{\gamma}=\frac{1}{2}\left(M_{\alpha}\right)_{b}^{c}\left(\sigma_{c}^{b}\right)_{\beta}^{\gamma},
$$

and the $\operatorname{Spin}\left(p_{+}, p_{-}\right)$invariance of the equations, the result follows.

Proposition 4. Assume that the bundle $P$ satisfies the torsion conditions of Proposition 3, and choose a connection $\omega$ so that the torsion $T$ of $\omega$ equals $T_{0}$. Suppose that there is an invertible $\operatorname{Spin}\left(p_{+}, p_{-}\right)$-invariant operator $D \in \operatorname{End}(V)$, such that for all $X \in \operatorname{so}\left(p_{+}, p_{-}\right), \varrho(X) D$ is symmetric. (If $\alpha$ of $(20)$ is -1 , we can take $D=C^{-1}$.) Then $\omega$ is flat.

Proof. From the Bianchi identities, we have

As

$$
\begin{aligned}
& d T^{a}+T^{b} \wedge \omega_{b}{ }^{a}=\tau^{b} \wedge{\Omega_{b}}^{a}, \\
& d T^{\alpha}+T^{\beta} \wedge \omega_{\beta}{ }^{\alpha}=\tau^{\beta} \wedge{\Omega_{\beta}}^{\alpha} .
\end{aligned}
$$

$$
T^{a}=\frac{1}{2} \tau^{\beta} \wedge \tau^{\alpha}\left(\gamma^{a} C^{-1}\right)_{\alpha \beta}
$$

and the other components of $T$ vanish, it follows that the left-hand sides of (51) and (52) vanish. Let us write $\Omega_{b}{ }^{a}$ as

$$
\Omega_{b}{ }^{a}=\frac{1}{2} \tau^{d} \wedge \tau^{c} \Omega_{c d b}{ }^{a}+\tau^{\delta} \wedge \tau^{c} \Omega_{c \delta b}{ }^{a}+\frac{1}{2} \tau^{\delta} \wedge \tau^{\gamma} \Omega_{\gamma \delta b}{ }^{a},
$$

and similarly for $\Omega_{\beta}{ }^{\alpha}$. From (51) follows

From (52) follows

$$
\begin{aligned}
& \Omega_{c d b}{ }^{a}+\Omega_{d b c}{ }^{a}+\Omega_{b c d}{ }^{a}=0, \\
& \Omega_{c \delta b}{ }^{a}-\Omega_{b \delta c}{ }^{a}=0, \\
& \Omega_{\gamma \delta b}{ }^{a}=0 .
\end{aligned}
$$

Because $\varrho$ is faithful, (58) implies that $\Omega_{c d b}{ }^{a}$ vanishes.

Let us use $D$ to lower the last index on $\Omega_{c \delta \beta}{ }^{\alpha}$. Then $\Omega_{c \delta \beta \alpha}$ is symmetric in $\beta$ and $\alpha$, and antisymmetric in $\delta$ and $\beta$. It follows that $\Omega_{c \delta \beta \alpha}$ vanishes, and so $\omega$ is flat. 
For four-dimensional Minkowski space, we have $\alpha=-1$. Proposition 4 shows that the assumption that $P$ is a first-order flat $\operatorname{Spin}\left(p_{+}, p_{-}\right)$bundle is too restrictive, as one would like to deal with nonflat geometries. We will also want to allow for a larger structure group than $\operatorname{Spin}\left(\mathrm{p}_{+}, p_{-}\right)$in order to deal with gauged extended supersymmetries. The correct assumption is as follows:

Definition 3. Let $K$ be a Lie group. Suppose that $V$ breaks up as $V=V^{\prime} \otimes V^{\prime \prime}$ and that

$$
\tilde{\varrho}=\varrho \otimes \varrho^{\prime}: \operatorname{Spin}\left(p_{+}, p_{-}\right) \times K \rightarrow \operatorname{End}\left(V^{\prime} \otimes V^{\prime \prime}\right)
$$

is a tensor product representation. ( $K$ is the symmetry group for an extended supersymmetry.) Let $\mathscr{S}$ be a subspace of End $\left(\mathbb{R}^{p}, V\right)$ which is $\operatorname{Spin}\left(p_{+}, p_{-}\right) \times K$ invariant. A superRiemannian structure bundle $P$ is given by a reduction of the frame bundle of $X$ to the subgroup

$$
G=\left(\operatorname{Spin}\left(p_{+}, p_{-}\right) \times K\right) \tilde{\times} \mathscr{S}
$$

which is first-order flat.

The reduction of the frame bundle to the structure group $G$ has the consequence that there is a well-defined subbundle $T^{\text {odd }}$ of the tangent bundle, given by the odd directions. In this sense a superRiemannian structure is like a foliation of an ordinary manifold. However, even for a flat superspace, instead of being integrable the distribution $T^{\text {odd }}$ is maximally nonintegrable. The requirement of first-order flatness has as a consequence that this will also be true for a supermanifold with a superRiemannian structure.

For simplicity, in the rest of this section we will only consider the case of unextended supersymmetry, i.e. $K=\{e\}$. The model $G$-geometry is given by the reduction (of the frame bundle of $\mathbb{R}^{p} \mid V$ to $G$ ) which contains the section $s$. At $s$, the torsion tensor again has only $\left(T_{0}\right)_{\alpha \beta}{ }^{a}=\left(\gamma^{a} C^{-1}\right)_{\alpha \beta}$ as a nonzero component.

Proposition 5. For $\operatorname{Spin}\left(p_{+}, p_{-}\right) \tilde{\times} \mathscr{S}$ acting on $W=\mathbb{R}^{p} \mid V, g^{(1)} \subseteq \mathbb{R}^{p} \otimes \mathscr{S}$. Necessary conditions for a tensor $T \in \operatorname{Hom}(W \wedge W, W)$ to lie in the same orbit in $H^{0,2}$ as $T_{0}$ are

$$
\begin{aligned}
& T_{\alpha \beta}{ }^{c}=\left(\gamma^{c} C^{-1}\right)_{\alpha \beta^{\prime}}, \\
& T_{\alpha \beta}{ }^{\gamma}-\frac{1}{2} T_{\alpha a c}\left(\sigma^{c a}\right)_{\beta}{ }^{\gamma}-\frac{1}{2} T_{\beta a c}\left(\sigma^{c a}\right)_{\alpha}{ }^{\gamma}=-\left(\gamma^{c} C^{-1}\right)_{\alpha \beta} X_{c}{ }^{\gamma} \\
& +\frac{1}{2} X_{a}^{\beta^{\prime}}\left(\gamma_{c} C^{-1}\right)_{\alpha \beta^{\prime}}\left(\sigma^{c a}\right)_{\beta}{ }^{\gamma}+\frac{1}{2} X_{a}^{\beta^{\prime}}\left(\gamma_{c} C^{-1}\right)_{\beta \beta^{\prime}}\left(\sigma^{c a}\right)_{\alpha}{ }^{\gamma}
\end{aligned}
$$

for some $X \in \mathscr{S}$.

If $\mathscr{S}=\operatorname{End}\left(\mathbb{R}^{p}, V\right)$ then these are also sufficient conditions.

Proof. With the notation of the proof of Proposition 3, we have

$$
\begin{aligned}
& S_{a b}{ }^{c}=\left(M_{a}\right)_{b}{ }^{c}-\left(M_{b}\right)_{a}{ }^{c}, \\
& S_{a b}{ }^{\gamma}=\left(M_{a}\right)_{b}{ }^{\gamma}-\left(M_{b}\right)_{a}{ }^{\gamma}, \\
& S_{a \beta}{ }^{c}=-\left(M_{\beta}\right)_{a}{ }^{c}, \\
& S_{a \beta}{ }^{\gamma}=\left(M_{a}\right)_{\beta}{ }^{\gamma}-\left(M_{\beta}\right)_{a}^{\gamma}, \\
& S_{\alpha \beta}{ }^{c}=0, \\
& S_{\alpha \beta}{ }^{\gamma}=\left(M_{\alpha}\right)_{\beta}{ }^{\gamma}+\left(M_{\beta}\right)_{\alpha}{ }^{\gamma} .
\end{aligned}
$$


Suppose that $M \in g^{(1)}$. From (64), $\left(M_{a}\right)_{b c}$ is symmetric in $a$ and $b$, and antisymmetric in $b$ and $c$, and so vanishes. Then $\left(M_{a}\right)_{\beta}{ }^{\gamma}$ also vanishes, and so from (67), $\left(M_{\beta}\right)_{a}^{\gamma}$ vanishes. From (66), $\left(M_{\beta}\right)_{a}{ }^{c}$ vanishes, and so $\left(M_{\beta}\right)_{\alpha}^{\gamma}$ vanishes. Thus $M \in \mathbb{R}^{p} \otimes \mathscr{S}$, and satisfies $\left(M_{a}\right)_{b}{ }^{\gamma}=\left(M_{b}\right)_{a}{ }^{\gamma}$.

To show that (62) and (63) are necessary conditions it suffices to show that both $\left\{g \cdot T_{0}: g \in G\right\}$ and $T_{0}+\operatorname{Im} \delta$ satisfy the conditions (62) and (63). For $T_{0}+\operatorname{Im} \delta$, this follows from (64-69). For $g \cdot T_{0}$, the fact that

$$
\left(g \cdot T_{0}\right)_{\alpha \beta}^{c}=\left(\gamma^{c} C^{-1}\right)_{\alpha \beta}
$$

is satisfied follows from $\operatorname{Spin}\left(p_{+}, p_{-}\right)$invariance of $\left(\gamma^{c} C^{-1}\right)_{\alpha \beta}$. It now suffices to consider $g=1 \tilde{\times} Z$ with $Z \in \mathscr{S}$. If one writes out the left-hand side of (63) for $T=g \cdot T_{0}$, one finds exactly the right-hand side of (63) with $X=Z$.

If $\mathscr{S}=$ End $\left(\mathbb{R}^{p}, V\right)$ and the conditions (62) and (63) are satisfied, put $g=1 \tilde{\times} X$, where $X$ is given on the right-hand side of (63). If we put

$$
S=T-g \cdot T_{\mathrm{o}},
$$

then $S_{\alpha \beta}{ }^{c}$ and $S_{\alpha \beta}{ }^{\gamma}-\frac{1}{2} S_{\alpha a c}\left(\sigma^{c a}\right)_{\beta}{ }^{\gamma}-\frac{1}{2} S_{\beta a c}\left(\sigma^{c a}\right)_{\alpha}{ }^{\gamma}$ vanish. It suffices to show $S=\delta(M)$ for some

Take

$$
M \in W^{*} \otimes\left(\operatorname{so}\left(p_{+}, p_{-}\right) \oplus \operatorname{End}\left(\mathbb{R}^{p}, V\right)\right)
$$

$$
\begin{aligned}
& \left(M_{a}\right)_{b c}=\frac{1}{2}\left(S_{a b c}-S_{b c a}+S_{c a b}\right), \\
& \left(M_{a}\right)_{\beta}{ }^{\gamma}=\frac{1}{2}\left(M_{a}\right)_{b c}\left(\sigma^{c b}\right)_{\beta}{ }^{\gamma}, \\
& \left(M_{a}\right)_{b}{ }^{\gamma}=\frac{1}{2} S_{a b}{ }^{\gamma}, \\
& \left(M_{\beta}\right)_{a}^{\gamma}=\left(M_{a}\right)_{\beta}^{\gamma}-S_{a \beta}^{\gamma}, \\
& \left(M_{\beta}\right)_{a}^{c}=-S_{a \beta}{ }^{c}, \\
& \left(M_{\beta}\right)_{\alpha}^{\gamma}=\frac{1}{2}\left(M_{\beta}\right)_{a}{ }^{c}\left(\sigma_{c}{ }^{a}\right)_{\alpha}{ }^{\gamma} .
\end{aligned}
$$

In the physics literature one does not talk about $\operatorname{Spin}\left(p_{+}, p_{-}\right) \tilde{\times} \mathscr{S}$ structures, but instead about $\operatorname{Spin}\left(p_{+}, p_{-}\right)$structures. To make the comparison, suppose that we have a superRiemannian structure bundle. If we write the torsion equations, we can transfer the $\mathscr{S}$ part of the connection to the other side of the equation and consider that we have torsion equations for a $\operatorname{Spin}\left(p_{+}, p_{-}\right)$geometry, but now with a nonflat torsion. Thus the first-order flatness of a $\operatorname{Spin}\left(p_{+}, p_{-}\right) \tilde{\times} \mathscr{S}$ structure becomes translated into the nonvanishing of certain components of the torsion tensor of a $\operatorname{Spin}\left(p_{+}, p_{-}\right)$structure.

To formalize this, suppose that we have a superRiemannian structure bundle $P$. We can then find a local section $\sigma$ of $P$ such that along $\sigma, T \in T_{0}+\operatorname{Im} \delta$.

Definition 4. A superRiemannian geometry is a reduction of $P$ to a $\operatorname{Spin}\left(p_{+}, p_{-}\right)$ subbundle $Q$ such that $T \in T_{0}+\operatorname{Im} \delta$ on $Q$.

Proposition 6. There is a connection $\omega$ on $Q$ for which the only nonzero components of the torsion $T$ are

$$
T_{\alpha \beta}^{c}=\left(\gamma^{c} C^{-1}\right)_{\alpha \beta}, T_{a b}^{\alpha} \text { and } T_{a \beta}^{\alpha}
$$

If these components are given then $\omega$ is unique. 
Proof. We have that there is a $G$-connection $\omega^{\prime}$ on $P$ such that when restricted to $Q$,

$$
\begin{aligned}
& \left(T_{0}\right)^{a}=d \tau^{a}+\tau^{b} \wedge \omega_{b}^{\prime a}, \\
& \left(T_{0}\right)^{\alpha}=d \tau^{\alpha}+\tau^{\beta} \wedge \omega_{\beta}^{\prime \alpha}+\tau^{a} \wedge \omega_{a}^{\prime \alpha} .
\end{aligned}
$$

Define a $\operatorname{Spin}\left(p_{+}, p_{-}\right)$connection $\omega$ by

Then

$$
\omega_{b}^{a}=\omega_{b}^{\prime a}, \omega_{\beta}^{\alpha}=\omega_{\beta}^{\prime \alpha}=\omega_{b}^{\prime a}\left(\sigma_{a}^{b}\right)_{\beta}^{\alpha} .
$$

$$
\begin{aligned}
T^{a}=d \tau^{a}+\tau^{b} \wedge \omega_{b}{ }^{a} & =\left(T_{0}\right)^{a}, \\
T^{\alpha}=d \tau^{\alpha}+\tau^{\beta} \wedge \omega_{\beta}{ }^{\alpha} & =\left(T_{0}\right)^{\alpha}-\tau^{a} \wedge \omega_{a}^{\prime \alpha} \\
& =\left(T_{0}\right)^{\alpha}-\tau^{a} \wedge \tau^{b} \omega_{b a}^{\prime}{ }^{\alpha}-\tau^{a} \wedge \tau^{\beta} \omega_{\beta a}^{\prime}{ }^{\alpha} .
\end{aligned}
$$

The uniqueness of $\omega$ follows as in the analogous uniqueness of the Levi-Cività connection.

Proposition 7 (Dragon's theorem [Dr]). Under the hypotheses of Proposition 4, the curvature $\Omega$ of $\omega$ can be written explicitly in terms of $T$ and $\nabla T$.

Proof. The left-hand side of (51) and (52) can be written in terms of $T$ and $\nabla T$. The proposition follows from the proof of Proposition 4.

\section{Examples}

We will show that the above definition of a superRiemannian geometry gives the minimal supergravity theories in 2, 3, 4, and 6 dimensions. We will assume that we have a superRiemannian structure bundle for the group $\left(\operatorname{Spin}\left(p_{+}, p_{-}\right) \times K\right) \tilde{\times} \mathscr{S}$ and find the consequences of the torsion equations of a supergeometry. We will do this by writing the torsion equations for a first-order flat $\left(\operatorname{Spin}\left(p_{+}, p_{-}\right) \times K\right) \tilde{\times} \mathscr{S}$ structure in the form of torsion equations for a non-first-order flat $\operatorname{Spin}\left(p_{+}, p_{-}\right) \times K$ structure. We will also look at the different geometries which arise from different choices of the subspace $\mathscr{S}$.

\section{A. One Dimension}

Let $V$ be $\mathbb{R}^{1}$. The torsion equations become

$$
\begin{aligned}
& d \tau^{x}=-\tau^{\theta} \wedge \tau^{\theta}, \\
& d \tau^{\theta}=T \tau^{\theta} \wedge \tau^{x}
\end{aligned}
$$

for some even torsion component $T$. Taking the exterior derivative of (84) gives

$$
0=-2 T \tau^{\theta} \wedge \tau^{\theta} \wedge \tau^{x}
$$

and so $T=0$. By the super Poincaré lemma [Ma], we can locally write $\tau^{\theta}$ as $d \eta$, where $\eta$ is an odd 0 -form. Then (84) becomes

$$
d\left(\tau^{x}-\eta d \eta\right)=0,
$$


and so locally we can find an even 0 -form $z$ so that

$$
\tau^{x}=d z+\eta d \eta, \quad \tau^{\theta}=d \eta
$$

Thus, a one-dimensional supergeometry is locally equivalent to the flat supergeometry

$$
\tau^{x}=d x+\theta d \theta, \quad \tau^{\theta}=d \theta
$$

\section{B. Two Dimensions}

I. (1,0) Supergeometry. Let us take $S O\left(p_{+}, p_{-}\right)=S O(1,1) \cong \mathbb{R}^{+}$and $V=\mathbb{R}$. (In the two-dimensional case we will define $\operatorname{Spin}(1,1)$ to be $\mathbb{R}^{+}$, with the "double cover": $\operatorname{Spin}(1,1) \rightarrow S O(1,1)$ given by $c(x)=x^{2}$.) Let $x^{ \pm}$denote the light cone coordinates $x^{ \pm}=\left(x^{0} \pm x^{1}\right) / \sqrt{2}$ and let $\theta$ denote the odd coordinate. Take $\mathscr{S}$ to be the subspace of End $\left(\mathbb{R}^{p}, V\right)$ which vanishes on $\partial_{x^{-}}$, i.e. has the matrix form $M_{+}{ }^{\theta}$. The torsion equations become

$$
\begin{aligned}
& d \tau^{+}+\tau^{+} \wedge \omega_{+}{ }^{+}=-\tau^{\theta} \wedge \tau^{\theta}, \\
& d \tau^{-}+\tau^{-} \wedge \omega_{-}^{-}=0, \\
& d \tau^{\theta}+\tau^{\theta} \wedge \omega_{\theta}{ }^{\theta}=S \tau^{+} \wedge \tau^{\theta}+T \tau^{+} \wedge \tau^{-},
\end{aligned}
$$

where $S$ is an even function, $T$ is an odd function and

Proposition 8. $S=0$.

$$
\omega_{+}{ }^{+}=-\omega_{-}{ }^{-}=2 \omega_{\theta}{ }^{\theta} \text {. }
$$

Proof. The exterior derivatives of (90) and (91) give

$$
\begin{aligned}
& \tau^{+} \wedge \Omega_{+}{ }^{+}=\tau^{+} \wedge\left(2 S \tau^{\theta} \wedge \tau^{\theta}-2 T \tau^{\theta} \wedge \tau^{-}\right), \\
& \tau^{-} \wedge \Omega_{-}{ }^{-}=0 .
\end{aligned}
$$

For these to be consistent, $S$ must vanish.

Thus the above choice of the subspace $\mathscr{S}$ gives the heterotic geometry described by Moore and Nelson [MN]. The Bianchi identities give

where

$$
\Omega_{\theta}{ }^{\theta}=\frac{1}{2} \Omega_{+}{ }^{+}=-T \tau^{\theta} \wedge \tau^{-}+\left(\nabla_{\theta} T\right) \tau^{+} \wedge \tau^{-},
$$

$$
\nabla_{\theta} T=e_{\theta} T-T \omega_{\theta \theta}{ }^{\theta}
$$

II. (1,1) Supersymmetry. Let us take $S O\left(p_{+}, p_{-}\right)=S O(2)$ and $V=\mathbb{R}^{2}$ with the spinor representation of Spin (2), the double covering of $S O(2)$. Let both $\mathbb{R}^{p}=\mathbb{R}^{2}$ and $V$ have the standard complex structure, and let $\mathscr{S}$ be the space of complex-linear maps from $\mathbb{R}^{2}$ to $V$. It is convenient to complexify both $\mathbb{R}^{2}$ and $V$, in which case the torsion equations become

$$
\begin{aligned}
& d \tau^{z}+\tau^{z} \wedge \omega_{z}{ }^{z}=-\tau^{\theta} \wedge \tau^{\theta} \\
& d \tau^{\bar{z}}+\tau^{\bar{z}} \wedge \omega_{\bar{z}}^{\bar{z}}=-\tau^{\bar{\theta}} \wedge \tau^{\bar{\theta}}, \\
& d \tau^{\theta}+\tau^{\theta} \wedge \omega_{\theta}{ }^{\theta}=S \tau^{z} \wedge \tau^{\theta}+T \tau^{z} \wedge \tau^{\bar{\theta}}+U \tau^{z} \wedge \tau^{\bar{z}}, \\
& d \tau^{\bar{\theta}}+\tau^{\bar{\theta}} \wedge \omega_{\bar{\theta}}^{\bar{\theta}}=\bar{S} \tau^{\bar{z}} \wedge \tau^{\bar{\theta}}+\bar{T} \tau^{\bar{z}} \wedge \tau^{\theta}+\bar{U} \tau^{\bar{z}} \wedge \tau^{z},
\end{aligned}
$$


where $\tau^{\bar{z}}$ is the complex conjugate of $\tau^{z}, \bar{T}$ is the complex conjugate of $T$, etc., $\omega_{z}{ }^{z}$ is purely imaginary and

$$
\omega_{z}^{z}=2 \omega_{\theta}{ }^{\theta} \text {. }
$$

Proposition 9. $S=0, T+\bar{T}=0$ and $U=\nabla_{\bar{\theta}} T$, where

$$
\nabla_{\bar{\theta}} T=e_{\bar{\theta}} T-\omega_{\bar{\theta} z}{ }^{z} T \text {. }
$$

Proof. The exterior derivative of (98) gives

and so

$$
\tau^{z} \wedge \Omega_{z}{ }^{z}=\tau^{z} \wedge\left(2 S \tau^{\theta} \wedge \tau^{\theta}+2 T \tau^{\theta} \wedge \tau^{\bar{\theta}}-2 U \tau^{\theta} \wedge \tau^{\bar{z}}\right)
$$

Similarly,

$$
\Omega_{z}{ }^{z}=2 S \tau^{\theta} \wedge \tau^{\theta}+2 T \tau^{\theta} \wedge \tau^{\bar{\theta}}-2 U \tau^{\theta} \wedge \tau^{\bar{z}} \bmod \tau^{z}
$$

Because

$$
\Omega_{\bar{z}}^{\bar{z}}=2 \bar{S} \tau^{\bar{\theta}} \wedge \tau^{\bar{\theta}}+2 \bar{T} \tau^{\bar{\theta}} \wedge \tau^{\theta}-2 \bar{U} \tau^{\bar{\theta}} \wedge \tau^{z} \bmod \tau^{\bar{z}}
$$

$$
\Omega_{z}{ }^{2}+\Omega_{\bar{z}}^{\bar{z}}=0
$$

it follows that $S=0$ and $T+\bar{T}=0$. Using this information, the exterior derivative of (100) gives

$$
\begin{aligned}
\tau^{\theta} \wedge \Omega_{\theta}{ }^{\theta}= & \tau^{\theta} \wedge\left[\left(\nabla_{\theta} U-T^{2}\right) \tau^{z} \wedge \tau^{\bar{z}}+T \tau^{\theta} \wedge \tau^{\bar{\theta}}+\nabla_{\theta} T \tau^{z}\right. \\
& \left.\wedge \tau^{\bar{\theta}}+U \tau^{\bar{z}} \wedge \tau^{\theta}\right]+\left(\nabla_{\bar{\theta}} T-U\right) \tau^{z} \\
& \wedge \tau^{\bar{\theta}} \wedge \tau^{\bar{\theta}}+\left(\nabla_{\bar{\theta}} U-\nabla_{\bar{z}} T\right) \tau^{\bar{\theta}} \wedge \tau^{z} \wedge \tau^{\bar{z}}
\end{aligned}
$$

Thus $U=\nabla_{\bar{\theta}} T$.

Thus the restriction to the subspace $\mathscr{S}$ gives the $N=1$ supergeometry described by Howe [Ho1]. The Bianchi identities give

$$
\begin{aligned}
\Omega_{\theta}{ }^{\theta}= & \left(\nabla_{\theta} \nabla_{\bar{\theta}} T-T^{2}\right) \tau^{z} \wedge \tau^{\bar{z}}+T \tau^{\theta} \wedge \tau^{\bar{\theta}}+\nabla_{\theta} T \tau^{z} \\
& \wedge \tau^{\bar{\theta}}+\nabla_{\bar{\theta}} T \tau^{\bar{z}} \wedge \tau^{\theta} .
\end{aligned}
$$

Suppose that we take $\mathscr{S}$ to be the space of all linear maps from $\mathbb{R}^{2}$ to $V$. The torsion equations become

$$
\begin{gathered}
d \tau^{z}+\tau^{z} \wedge \omega_{z}{ }^{z}=-\tau^{\theta} \wedge \tau^{\theta}, \\
d \tau^{\bar{z}}+\tau^{\bar{z}} \wedge \omega_{\bar{z}}^{\bar{z}}=-\tau^{\bar{\theta}} \wedge \tau^{\bar{\theta}}, \\
d \tau^{\theta}+\tau^{\theta} \wedge \omega_{\theta}{ }^{\theta}=S \tau^{z} \wedge \tau^{\theta}+T \tau^{z} \wedge \tau^{\bar{\theta}}+U \tau^{z} \\
\wedge \tau^{\bar{z}}+Q \tau^{\bar{z}} \wedge \tau^{\theta}+R \tau^{\bar{z}} \wedge \tau^{\bar{\theta}}, \\
d \tau^{\bar{\theta}}+\tau^{\bar{\theta}} \wedge \omega_{\bar{\theta}}^{\bar{\theta}}=\bar{S} \tau^{\bar{z}} \wedge \tau^{\bar{\theta}}+\bar{T} \tau^{z} \wedge \tau^{\theta}+\bar{U} \tau^{\bar{z}} \\
\wedge \tau^{z}+\bar{Q} \tau^{z} \wedge \tau^{\bar{\theta}}+\bar{R} \tau^{z} \wedge \tau^{\theta} .
\end{gathered}
$$

The exterior derivative of (110) gives

$$
\begin{aligned}
\tau^{z} \wedge \Omega_{z}{ }^{z}= & \tau^{z} \wedge\left(2 S \tau^{\theta} \wedge \tau^{\theta}+2 T \tau^{\theta} \wedge \tau^{\bar{\theta}}-2 U \tau^{\theta} \wedge \tau^{\bar{z}}\right) \\
& -2 Q \tau^{\theta} \wedge \tau^{\bar{z}} \wedge \tau^{\theta}-2 R \tau^{\theta} \wedge \tau^{\bar{z}} \wedge \tau^{\bar{\theta}} .
\end{aligned}
$$

Thus $Q=R=0$, and we are back to the case of $\mathscr{S}$ consisting of complex-linear maps. 
III. (2,0) Supersymmetry. Let us take $S O\left(p_{+}, p_{-}\right)=S O(1,1), \quad V=\mathbb{C}$ and $K=S O(2)$. Let $x^{ \pm}$denote the light cone coordinates $x^{ \pm}=\left(x^{0} \pm x^{1}\right) / \sqrt{2}$ and let $\theta$ denote the odd coordinate, with complex conjugate $\bar{\theta}$. Take $\mathscr{S}$ to be the $\mathbb{C}^{1}$ subspace of End $\left(\mathbb{R}^{p}, V\right)$ which vanishes on $\partial_{x^{-}}$, i.e. has the form $M_{+}{ }^{\theta}$. The torsion equations become

$$
\begin{aligned}
& d \tau^{+}+\tau^{+} \wedge \omega_{+}^{+}=-\tau^{\bar{\theta}} \wedge \tau^{\theta}, \\
& d \tau^{-}+\tau^{-} \wedge \omega_{-}^{-}=0, \\
& d \tau^{\theta}+\tau^{\theta} \wedge \omega_{\theta}{ }^{\theta}=R \tau^{+} \wedge \tau^{\bar{\theta}}+S \tau^{+} \wedge \tau^{\theta}+T \tau^{+} \wedge \tau^{-}, \\
& d \tau^{\bar{\theta}}+\tau^{\bar{\theta}} \wedge \omega_{\bar{\theta}}^{\bar{\theta}}=\bar{R} \tau^{+} \wedge \tau^{\theta}+\bar{S} \tau^{+} \wedge \tau^{\bar{\theta}}+\bar{T} \tau^{+} \wedge \tau^{-},
\end{aligned}
$$

where $R$ and $S$ are even functions, $T$ is an odd function and

$$
\operatorname{Re} \omega_{\theta}{ }^{\theta}=\frac{1}{2} \omega_{+}{ }^{+}=-\frac{1}{2} \omega_{-}{ }^{-}, \quad \omega_{\bar{\theta}}^{\bar{\theta}}=\left(\omega_{\theta}{ }^{\theta}\right)^{*} .
$$

$\bar{R}$ is the complex conjugate of $R$, etc. By using the freedom in $\operatorname{Im} \delta$ from redefining the connection, we can assume that $S=\bar{S}$.

Proposition 10. $R=S=0$ and $\nabla_{\bar{\theta}} T=0$, where

$$
\nabla_{\bar{\theta}} T=e_{\bar{\theta}} T-T \omega_{\bar{\theta} \theta}{ }^{\theta} .
$$

Proof. The exterior derivatives of (115) and (116) give

and

$$
\begin{gathered}
\tau^{+} \wedge \Omega_{+}{ }^{+}=\tau^{+} \wedge\left(R \tau^{\bar{\theta}} \wedge \tau^{\bar{\theta}}+\bar{R} \tau^{\theta} \wedge \tau^{\theta}+(S+\bar{S}) \tau^{\theta}\right. \\
\left.\wedge \tau^{\bar{\theta}}+T \tau^{-} \wedge \tau^{\bar{\theta}}+\bar{T} \tau^{-} \wedge \tau^{\theta}\right)
\end{gathered}
$$

For these to be consistent, $R$ and $S+\bar{S}$ must vanish. Thus

$$
R=S=0 \text {. }
$$

The exterior derivative of (117) gives

$$
\begin{aligned}
\tau^{\theta} \wedge \Omega_{\theta}{ }^{\theta}= & \tau^{\theta} \wedge\left(\nabla_{\theta} T \tau^{+} \wedge \tau^{-}+T \tau^{-} \wedge \tau^{\bar{\theta}}\right) \\
& +\nabla_{\bar{\theta}} T \tau^{+} \wedge \tau^{-} \wedge \tau^{\bar{\theta}}
\end{aligned}
$$

Thus $\nabla_{\bar{\theta}} T$ must vanish.

The curvature is given by

$$
\begin{aligned}
& \Omega_{\theta}{ }^{\theta}=\nabla_{\theta} T \tau^{+} \wedge \tau^{-}+T \tau^{-} \wedge \tau^{\bar{\theta}}, \\
& \Omega_{\bar{\theta}}{ }^{\bar{\theta}}=\left(\Omega_{\theta}{ }^{\theta}\right)^{*}, \\
& \Omega_{+}{ }^{+}=-\Omega_{-}{ }^{-}=\Omega_{\theta}{ }^{\theta}+\Omega_{\bar{\theta}}{ }^{\bar{\theta}} .
\end{aligned}
$$

$I V .(2,1)$ Supersymmetry. Let us take $S O\left(p_{+}, p_{-}\right)=S O(1,1), V=\mathbb{C} \oplus \mathbb{R}$ and $K=S O(2)$. Let $x^{ \pm}$denote the light cone coordinates $x^{ \pm}=\left(x^{0} \pm x^{1}\right) / \sqrt{2}$ and let $\theta$ and $\sigma$ denote the odd coordinates, where $\theta$ has complex conjugate $\bar{\theta}$. Take $\mathscr{S}$ to be the $\mathbb{C}^{1} \oplus \mathbb{R}$ subspace of End $\left(\mathbb{R}^{p}, V\right)$ which has the matrix form $M_{+}{ }^{\theta} \oplus M_{-}{ }^{\sigma}$. The 
torsion equations become

$$
\begin{aligned}
& d \tau^{+}+\tau^{+} \wedge \omega_{+}^{+}=-\tau^{\bar{\theta}} \wedge \tau^{\theta}, \\
& d \tau^{-}+\tau^{-} \wedge \omega_{-}^{-}=-\tau^{\sigma} \wedge \tau^{\sigma}, \\
& d \tau^{\theta}+\tau^{\theta} \wedge \omega_{\theta}{ }^{\theta}=R \tau^{+} \wedge \tau^{\bar{\theta}}+S \tau^{+} \wedge \tau^{\theta}+T \tau^{+} \wedge \tau^{-}+U \tau^{+} \wedge \tau^{\sigma}, \\
& d \tau^{\bar{\theta}}+\tau^{\bar{\theta}} \wedge \omega_{\bar{\theta}}^{\bar{\theta}}=\bar{R} \tau^{+} \wedge \tau^{\theta}+\bar{S} \tau^{+} \wedge \tau^{\bar{\theta}}+\bar{T} \tau^{+} \wedge \tau^{-}+\bar{U} \tau^{+} \wedge \tau^{\sigma}, \\
& d \tau^{\sigma}+\tau^{\sigma} \wedge \omega_{\sigma}{ }^{\sigma}=N \tau^{-} \wedge \tau^{\sigma}+P \tau^{-} \wedge \tau^{\theta}+\bar{P} \tau^{-} \wedge \tau^{\bar{\theta}}+Q \tau^{-} \wedge \tau^{+},
\end{aligned}
$$

where $R, S, U, N$, and $P$ are even functions, $T$ and $Q$ are odd functions, $N=N^{*}$, $Q=Q^{*}$ and

$$
\omega_{\sigma}{ }^{\sigma}=\operatorname{Re} \omega_{\theta}{ }^{\theta}=\frac{1}{2} \omega_{+}^{+}=-\frac{1}{2} \omega_{-}^{-}, \quad \omega_{\bar{\theta}}^{\bar{\theta}}=\left(\omega_{\theta}^{\theta}\right)^{*} .
$$

As for the $(2,0)$ geometry, we can assume $\operatorname{Im} S=0$.

Proposition 11. $R=N=S=0, \nabla_{\theta} P=0, T=-2 \nabla_{\sigma} \bar{P}, U=-2 \bar{P}, Q=-\left(\nabla_{\theta} \bar{P}+\nabla_{\bar{\theta}} P\right)$.

Proof. The exterior derivatives of (128) and (129) give

and

$$
\begin{aligned}
\tau^{+} \wedge \Omega_{+}{ }^{+}= & \tau^{+} \wedge\left(R \tau^{\bar{\theta}} \wedge \tau^{\bar{\theta}}+\bar{R} \tau^{\theta} \wedge \tau^{\theta}+(S+\bar{S}) \tau^{\theta} \wedge \tau^{\bar{\theta}}\right. \\
& \left.+T \tau^{-} \wedge \tau^{\bar{\theta}}+\bar{T} \tau^{-} \wedge \tau^{\theta}+U \tau^{\sigma} \wedge \tau^{\bar{\theta}}+\bar{U} \tau^{\theta} \wedge \tau^{\sigma}\right)
\end{aligned}
$$

$$
\tau^{-} \wedge \Omega_{-}^{-}=2 \tau^{-} \wedge\left(N \tau^{\sigma} \wedge \tau^{\sigma}+P \tau^{\sigma} \wedge \tau^{\theta}+\bar{P} \tau^{\sigma} \wedge \tau^{\bar{\theta}}-Q \tau^{\sigma} \wedge \tau^{+}\right)
$$

For these to be consistent, $R, S+\bar{S}, U+2 P$ and $N$ must vansih. Thus $S=0$. We have

$$
\begin{aligned}
\Omega_{+}{ }^{+}= & T \tau^{-} \wedge \tau^{\bar{\theta}}+\bar{T} \tau^{-} \wedge \tau^{\theta}-2 \bar{P} \tau^{\sigma} \wedge \tau^{\bar{\theta}}-2 P \tau^{\sigma} \wedge \tau^{\theta} \\
& -2 Q \tau^{\sigma} \wedge \tau^{+}\left(\bmod \tau^{+} \wedge \tau^{-}\right) .
\end{aligned}
$$

The exterior derivative of (130) gives

Thus

$$
\begin{aligned}
\tau^{\theta} \wedge \Omega_{\theta}{ }^{\theta}= & \tau^{\theta} \wedge\left(\left(\nabla_{\theta} T-2 P \bar{P}\right) \tau^{+} \wedge \tau^{-}+T \tau^{-} \wedge \tau^{\bar{\theta}}-2 \nabla_{\theta} \bar{P}_{\tau^{+} \wedge \tau^{\sigma}}\right. \\
& \left.-2 \bar{P}_{\tau^{\sigma}} \wedge \tau^{\bar{\theta}}\right)+\left(\nabla_{\bar{\theta}} T-2 \bar{P}^{2}\right) \tau^{+} \wedge \tau^{-} \wedge \tau^{\bar{\theta}} \\
& +\left(\nabla_{\sigma} T+2 \nabla_{-} \bar{P}\right) \tau^{\sigma} \wedge \tau^{+} \wedge \tau^{-}-2 \nabla_{\bar{\theta}} \bar{P} \tau^{\sigma} \wedge \tau^{\bar{\theta}} \wedge \tau^{+} \\
& -\left(2 \nabla_{\sigma} \bar{P}+T\right) \tau^{+} \wedge \tau^{\sigma} \wedge \tau^{\sigma}
\end{aligned}
$$

The complex conjugate of (139) gives

$$
\begin{aligned}
\Omega_{\bar{\theta}}^{\bar{\theta}}= & -\left(2 \nabla_{\bar{\theta}} \nabla_{\sigma} P+2 \bar{P} P\right) \tau^{+} \wedge \tau^{-}-2 \nabla_{\sigma} P \tau^{-} \wedge \tau^{\theta} \\
& -2 \nabla_{\bar{\theta}} P \tau^{+} \wedge \tau^{\sigma}-2 P \tau^{\sigma} \wedge \tau^{\theta} .
\end{aligned}
$$

Using the fact that

$$
\Omega_{+}{ }^{+}=\Omega_{\theta}{ }^{\theta}+\Omega_{\bar{\theta}}^{\bar{\theta}}
$$


we obtain

$$
Q=-\left(\nabla_{\theta} \bar{P}+\nabla_{\bar{\theta}} P\right)
$$

The exterior derivative of (132) gives no further information.

$V .(2,2)$ Supersymmetry. Let us take $S O\left(p_{+}, p_{-}\right)=S O(2), V=\mathbb{C}^{2}$, each $\mathbb{C}$ with the spinor representation of Spin (2), the double covering of $S O(2)$, and $K=\mathbb{C}^{*}$. Let $\mathscr{S}$ be the space of complex-linear maps from $\mathbb{R}^{2}$ to $V$. The torsion equations become

$$
\begin{aligned}
& d \tau^{z}+\tau^{z} \wedge \omega_{z}{ }^{z}=-\tau^{\theta_{1}} \wedge \tau^{\theta_{2}}, \\
& d \tau^{\theta_{1}}+\tau^{\theta_{1}} \wedge \omega_{\theta_{1}}^{\theta_{1}}=S_{11} \tau^{z} \wedge \tau^{\theta_{1}}+S_{12} \tau^{z} \wedge \tau^{\theta_{2}}+T_{11} \tau^{z} \\
& \wedge \tau^{\bar{\theta}_{1}}+T_{12} \tau^{z} \wedge \tau^{\bar{\theta}_{2}}+U_{1} \tau^{z} \wedge \tau^{\bar{z}},
\end{aligned}
$$

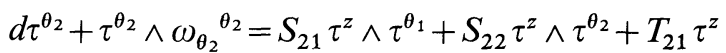

$$
\begin{aligned}
& \wedge \tau^{\bar{\theta}_{1}}+T_{22} \tau^{z} \wedge \tau^{\bar{\theta}_{2}}+U_{2} \tau^{z} \wedge \tau^{\bar{z}},
\end{aligned}
$$

along with the complex conjugates, where

$$
\omega_{\theta_{1}}^{\theta_{1}}=\omega_{\theta_{2}}^{\theta_{2}}=\omega_{z}{ }^{z}, \quad \omega_{z}{ }^{z}+\omega_{\bar{z}}^{\bar{z}}=0 .
$$

In this case we can use the freedom in $\operatorname{Im} \delta$ [for the structure group $\operatorname{Spin}(2) \times \mathbb{C}^{*}$ ] to set $S_{11}=S_{22}$.

\section{Proposition 12.}

$$
\begin{aligned}
& S_{11}=S_{12}=S_{21}=S_{22}=0, \\
& T_{22}+\overline{T_{11}}=T_{12}+\overline{T_{12}}=T_{21}+\overline{T_{21}}=0, \\
& \nabla_{\theta_{2}} T_{12}=\nabla_{\theta_{2}} T_{11}=\nabla_{\theta_{1}} T_{22}=\nabla_{\theta_{1}} T_{21}=0, \\
& U_{1}=\nabla_{\overline{\theta_{2}}} T_{11}+\nabla_{\overline{\theta_{1}}} T_{12}, \quad U_{2}=\nabla_{\overline{\theta_{1}}} T_{22}+\nabla_{\overline{\theta_{2}}} T_{21} .
\end{aligned}
$$

Proof The exterior derivative of (143) gives

$$
\begin{aligned}
\tau^{z} \wedge \Omega_{z}{ }^{z}= & \tau^{z} \wedge\left(S_{21} \tau^{\theta_{1}} \wedge \tau^{\theta_{1}}+S_{22} \tau^{\theta_{1}} \wedge \tau^{\theta_{2}}+T_{21} \tau^{\theta_{1}}\right. \\
& \wedge \tau^{\overline{\theta_{1}}}+T_{22} \tau^{\theta_{1}} \wedge \tau^{\overline{\theta_{2}}}+U_{2} \tau^{\bar{z}} \wedge \tau^{\theta_{1}}+S_{11} \tau^{\theta_{1}} \\
& \wedge \tau^{\theta_{2}}+S_{12} \tau^{\theta_{2}} \wedge \tau^{\theta_{2}}+T_{11} \tau^{\overline{\theta_{1}}} \wedge \tau^{\theta_{2}}+T_{12} \tau^{\overline{\theta_{2}}} \\
& \left.\wedge \tau^{\theta_{2}}+U_{1} \tau^{\bar{z}} \wedge \tau^{\theta_{2}}\right) .
\end{aligned}
$$

Along with the complex conjugate equation, and the fact that $\Omega_{z}{ }^{2}+\left(\Omega_{z}{ }^{z}\right)^{*}=0$, we obtain

$$
\begin{aligned}
& S_{11}=S_{12}=S_{21}=S_{22}=0, \\
& T_{22}+\overline{T_{11}}=T_{12}+\overline{T_{12}}=T_{21}+\overline{T_{21}}=0,
\end{aligned}
$$


and

$$
\begin{aligned}
\Omega_{z}{ }^{z}= & T_{21} \tau^{\theta_{1}} \wedge \tau^{\overline{\theta_{1}}}-\overline{T_{11}} \tau^{\theta_{1}} \wedge \overline{\theta^{\theta_{2}}}+T_{11} \tau^{\theta_{2}} \wedge \overline{\theta^{\theta_{1}}} \\
& +T_{12} \tau^{\theta_{2}} \wedge \tau^{\overline{\theta_{2}}}-U_{1} \tau^{\theta_{2}} \wedge \tau^{\bar{z}}-U_{2} \tau^{\theta_{1}} \wedge \tau^{\bar{z}}+\overline{U_{1}} \tau^{\overline{\theta_{2}}} \wedge \tau^{z} \\
& +\overline{U_{2}} \tau^{\overline{\theta_{1}}} \wedge \tau^{z}\left(\bmod \tau^{z} \wedge \tau^{\bar{z}}\right)
\end{aligned}
$$

Using this information, the exterior derivative of (144) gives

$$
\begin{aligned}
\tau^{\theta_{1}} \wedge \Omega_{\theta_{1}}^{\theta_{1}}= & \tau^{\theta_{1}} \wedge\left[\left(\nabla_{\theta_{1}} U_{1}+T_{11} \overline{T_{11}}+T_{12} \overline{T_{21}}\right) \tau^{z} \wedge \tau^{\bar{z}}\right. \\
& +\nabla_{\theta_{1}} T_{11} \tau^{z} \wedge \overline{\tau^{\theta_{1}}}+\nabla_{\theta_{1}} T_{12} \tau^{z} \wedge \overline{\tau^{\theta_{2}}}-U_{1} \tau^{\theta_{2}} \wedge \tau^{\bar{z}} \\
& \left.+T_{11} \tau^{\theta_{2}} \wedge \tau^{\overline{\theta_{1}}}+T_{12} \tau^{\theta_{2}} \wedge \tau^{\overline{\theta_{2}}}\right]+\left(\nabla_{\theta_{2}} U_{1}+T_{11} \overline{T_{12}}-T_{12} T_{11}\right) \tau^{z} \wedge \tau^{\bar{z}} \wedge \tau^{\theta_{2}} \\
& +\left(\nabla_{\overline{\theta_{1}}} U_{1}-\nabla_{\bar{z}} T_{11}\right) \tau^{z} \wedge \tau^{\bar{z}} \wedge \overline{\theta^{\overline{\theta_{1}}}}+\left(\nabla_{\overline{\theta_{2}}} U_{1}-\nabla_{\bar{z}} T_{12}\right) \tau^{z} \wedge \tau^{\bar{z}} \wedge \overline{\tau^{\overline{\theta_{2}}}} \\
& +\nabla_{\theta_{2}} T_{12} \tau^{z} \wedge \tau^{\theta_{2}} \wedge \tau^{\overline{\theta_{2}}}+\nabla_{\theta_{2}} T_{11} \tau^{z} \wedge \tau^{\theta_{2}} \wedge \tau^{\overline{\theta_{1}}}+\nabla_{\overline{\theta_{1}}} T_{11} \tau^{z} \wedge \overline{\tau^{\overline{\theta_{1}}}} \wedge \tau^{\overline{\theta_{1}}} \\
& +\nabla_{\overline{\theta_{2}}} T_{12} \tau^{z} \wedge \tau^{\overline{\theta_{2}}} \wedge \overline{\theta^{\theta_{2}}} \\
& +\left(-U_{1}+\nabla_{\overline{\theta_{2}}} T_{11}+\nabla_{\overline{\theta_{1}}} T_{12}\right) \tau^{z} \wedge \tau^{\overline{\theta_{1}}} \wedge \overline{\theta^{\theta_{2}}}
\end{aligned}
$$

Thus

and

$$
\nabla_{\theta_{2}} T_{12}=\nabla_{\theta_{2}} T_{11}=\nabla_{\overline{\theta_{1}}} T_{11}=\nabla_{\overline{\theta_{2}}} T_{12}=0
$$

$$
U_{1}=\nabla_{\overline{\theta_{2}}} T_{11}+\nabla_{\overline{\theta_{1}}} T_{12}
$$

Similarly, the exterior derivative of (145) gives

and

$$
\nabla_{\theta_{1}} T_{21}=\nabla_{\theta_{1}} T_{22}=\nabla_{\overline{\theta_{2}}} T_{22}=\nabla_{\overline{\theta_{1}}} T_{21}=0
$$

$$
U_{2}=\nabla_{\overline{\theta_{1}}} T_{22}+\nabla_{\overline{\theta_{2}}} T_{21} \text {. }
$$

Thus the restriction to the subspace $\mathscr{S}$ gives the $N=2$ supergeometry described by Howe and Papadopoulos [HP].

\section{Three Dimensions}

Let us take $S O\left(p_{+}, p_{-}\right)=S O(2,1), V=\mathbb{R}^{2}$ with the spinor representation of $\operatorname{Spin}(2,1)=S L(2, \mathbb{R})$. Following the notation of $[\mathrm{GGRS}]$, we will denote the matrix representation of an element $M$ of $s l(2, \mathbb{R})$ by $(M)_{ \pm}{ }^{ \pm}$with $\operatorname{Tr} M=0$. Using the invariant symplectic form $\varepsilon$ on $V$ to raise and lower indices, we can consider $M$ as an element of $S^{2}\left(\mathbb{R}^{2}\right)$, i.e. of the form $M_{ \pm \pm}$with $M_{\alpha \beta}=M_{\beta \alpha}$. We will also identify $\operatorname{sl}(2, \mathbb{R})$ with the Minkowski 3-space, to write an element of $\mathbb{R}^{3}$ in the form $P \in S^{2}\left(\mathbb{R}^{2}\right)$.

Let us first take $\mathscr{S}=\operatorname{End}\left(\mathbb{R}^{3}, V\right)$. The torsion equations become

$$
\begin{aligned}
& d \tau^{\alpha \beta}+\tau^{\gamma \beta} \wedge \omega_{\gamma}{ }^{\alpha}+\tau^{\alpha \gamma} \wedge \omega_{\gamma}{ }^{\beta}=-\tau^{\alpha} \wedge \tau^{\beta}, \\
& d \tau^{\alpha}+\tau^{\beta} \wedge \omega_{\beta}{ }^{\alpha}=\tau^{\gamma \delta} \wedge \tau^{\varepsilon} T_{\varepsilon, \delta \gamma}{ }^{\alpha}+\frac{1}{2} \tau^{\gamma \delta} \wedge \tau^{\varepsilon \phi} T_{\phi \varepsilon, \delta \gamma}{ }^{\alpha},
\end{aligned}
$$

where $\omega_{\alpha}{ }^{\beta}$ is $\operatorname{sl}(2, \mathbb{R})$-valued and $T_{\phi \varepsilon, \delta \gamma}{ }^{\alpha} \in \operatorname{Hom}\left(\Lambda^{2}\left(S^{2}\left(\mathbb{R}^{2}\right)\right), \mathbb{R}^{2}\right)$. It is known that the Bianchi identities imply that one can express the torsion and curvature in terms 
of a function $R$ and a tensor $G_{\alpha \beta \gamma}$ which is totally symmetric in its indices [GGRS]. A calculation gives

$$
\begin{gathered}
T_{\varepsilon, \delta \gamma}{ }^{\alpha}=R\left(\varepsilon_{\varepsilon \delta} \delta_{\gamma}{ }^{\alpha}+\varepsilon_{\varepsilon \gamma} \delta_{\varepsilon}{ }^{\alpha}\right) \\
T_{\phi \varepsilon, \delta \gamma}{ }^{\alpha}=\frac{1}{2}\left(\varepsilon_{\phi \delta} \delta_{\varepsilon}{ }^{\alpha} \nabla_{\gamma} R+\varepsilon_{\varepsilon \delta} \delta_{\phi}{ }^{\alpha} \nabla_{\gamma} R+\varepsilon_{\phi \gamma} \delta_{\varepsilon}^{\alpha} \nabla_{\delta} R+\varepsilon_{\varepsilon \gamma} \delta_{\phi}{ }^{\alpha} \nabla_{\delta} R\right. \\
-\varepsilon_{\delta \phi} \delta_{\gamma}{ }^{\alpha} \nabla_{\varepsilon} R-\varepsilon_{\gamma \phi} \delta_{\delta}{ }^{\alpha} \nabla_{\varepsilon} R-\varepsilon_{\delta \varepsilon} \delta_{\gamma}{ }^{\alpha} \nabla_{\phi} R-\varepsilon_{\gamma \varepsilon} \delta_{\delta}{ }^{\alpha} \nabla_{\phi} R \\
\left.+G_{\phi \delta}{ }^{\alpha} \varepsilon_{\varepsilon \gamma}+G_{\varepsilon \delta}{ }^{\alpha} \varepsilon_{\phi \gamma}+G_{\phi \gamma}{ }^{\alpha} \varepsilon_{\varepsilon \delta}+G_{\varepsilon \gamma}{ }^{\alpha} \varepsilon_{\phi \delta}\right)
\end{gathered}
$$

with the constraint

$$
\nabla_{\alpha} G_{\beta \gamma}{ }^{\alpha}+\nabla_{\beta} \nabla_{\gamma} R+\nabla_{\gamma} \nabla_{\beta} R=0
$$

There are two $S L(2, \mathbb{R})$-invariant subspaces of $\operatorname{End}\left(\mathbb{R}^{3}, V\right)=\operatorname{End}\left(S^{2}(V), V\right)$, namely

and

$$
\begin{gathered}
\mathscr{S}_{1}=\left\{M \in \operatorname{End}\left(S^{2}(V), V\right): \text { for all } v \in V, \operatorname{Tr} M_{v}=0,\right. \text { where } \\
\left.M_{v}: V \rightarrow V \text { is given by } M_{v}(w)=M(v \otimes w+w \otimes v)\right\}
\end{gathered}
$$

$$
\begin{aligned}
\mathscr{S}_{2}= & \left\{M \in \operatorname{End}\left(S^{2}(V), V\right): \exists z \in V^{*}\right. \text { such that } \\
& M(v, w)=z(v) w+z(w) v\} .
\end{aligned}
$$

Proposition 13. The supergeometry corresponding to $\mathscr{S}_{1}$ has vanishing $R$.

Proof. In matrix form,

$$
\mathscr{S}_{1}=\left\{M \in \operatorname{End}\left(S^{2}(V), V\right): M_{\alpha \beta}{ }^{\beta}=0\right\} .
$$

Thus the torsions must be writable as

where

$$
T_{\varepsilon, \delta \gamma}{ }^{\alpha}=\left(M_{\varepsilon}\right)_{\delta \gamma}{ }^{\alpha} \text { and } T_{\phi \varepsilon, \delta \gamma}{ }^{\alpha}=\left(M_{\phi \varepsilon}\right)_{\delta \gamma}{ }^{\alpha}-\left(M_{\delta \gamma}\right)_{\phi \varepsilon}{ }^{\alpha} \text {, }
$$

$$
\left(M_{\varepsilon}\right)_{\delta \alpha}^{\alpha}=0 \text { and }\left(M_{\phi \varepsilon}\right)_{\delta \alpha}^{\alpha}=0 .
$$

The condition $\left(T_{\varepsilon}\right)_{\delta \alpha}{ }^{\alpha}=0$ gives $R=0$. Let us guess that $\left(M_{\phi \varepsilon}\right)_{\delta \gamma \alpha}$ has the form

$$
\begin{aligned}
\left(M_{\phi \varepsilon}\right)_{\delta \gamma \alpha}= & \varepsilon_{\phi \delta} P_{\varepsilon \gamma \alpha}+\varepsilon_{\varepsilon \delta} P_{\phi \gamma \alpha}+\varepsilon_{\phi \gamma} P_{\varepsilon \delta \alpha}+\varepsilon_{\varepsilon \gamma} P_{\phi \delta \alpha} \\
& +\varepsilon_{\phi \alpha} P_{\varepsilon \delta \gamma}+\varepsilon_{\varepsilon \alpha} P_{\phi \delta \gamma}
\end{aligned}
$$

for some symmetric tensor $P$. This has the required symmetry that $\left(M_{\phi \varepsilon}\right)_{\delta \gamma \alpha}$ be symmetric in both $(\phi \varepsilon)$ and $(\delta \gamma \alpha)$. Then

$$
\begin{aligned}
T_{\phi \varepsilon, \delta \gamma \alpha}= & 2 \varepsilon_{\phi \delta} P_{\varepsilon \gamma \alpha}+2 \varepsilon_{\varepsilon \delta} P_{\phi \gamma \alpha}+2 \varepsilon_{\phi \gamma} P_{\varepsilon \delta \alpha}+2 \varepsilon_{\varepsilon \gamma} P_{\phi \delta \alpha} \\
& +\varepsilon_{\phi \alpha} P_{\varepsilon \delta \gamma}+\varepsilon_{\varepsilon \alpha} P_{\phi \delta \gamma}-\varepsilon_{\delta \alpha} P_{\gamma \phi \varepsilon}-\varepsilon_{\gamma \alpha} P_{\delta \phi \varepsilon} .
\end{aligned}
$$

One can check that if $P=G / 5$ this equals $\frac{1}{2}\left(G_{\phi \delta \alpha} \varepsilon_{\varepsilon \gamma}+G_{\varepsilon \delta \alpha} \varepsilon_{\phi \gamma}+G_{\phi \gamma \alpha} \varepsilon_{\varepsilon \delta}+G_{\varepsilon \gamma \alpha} \varepsilon_{\phi \delta}\right)$. Note that $G$ must still satisfy $\nabla_{\alpha} G_{\beta \gamma}{ }^{\alpha}=0$.

Proposition 14. The supergeometry corresponding to $\mathscr{S}_{2}$ is the same as the supergeometry corresponding to $\mathscr{S}=\mathrm{End}\left(\mathbb{R}^{3}, V\right)$.

Proof. In matrix form

$$
\mathscr{S}_{2}=\left\{M \in \operatorname{End}\left(S^{2}(V), V\right): M_{\delta \gamma}{ }^{\alpha}=Z_{\delta} \delta_{\gamma}{ }^{\alpha}+Z_{\gamma} \delta_{\delta}{ }^{\alpha} \text { for some } Z\right\} \text {. }
$$


Thus the torsions must be writable as

and

$$
T_{\varepsilon, \delta \gamma}{ }^{\alpha}=\left(Z_{\varepsilon}\right)_{\delta} \delta_{\gamma}{ }^{\alpha}+\left(Z_{\varepsilon}\right)_{\gamma} \delta_{\delta}^{\alpha}
$$

$$
T_{\phi \varepsilon, \delta \gamma}{ }^{\alpha}=\left(Z_{\phi \varepsilon}\right)_{\delta} \delta_{\gamma}{ }^{\alpha}+\left(Z_{\phi \varepsilon}\right)_{\gamma} \delta_{\delta}^{\alpha}-\left(Z_{\delta \gamma}\right)_{\phi} \delta_{\varepsilon}^{\alpha}-\left(Z_{\delta \gamma}\right)_{\varepsilon} \delta_{\phi}^{\alpha} \text {. }
$$

We can do this by putting $\left(Z_{\varepsilon}\right)_{\delta}$ to be $R \varepsilon_{\varepsilon \delta}$ and $\left(Z_{\phi \varepsilon}\right)_{\delta}$ to be $-\frac{1}{2}\left(\varepsilon_{\delta \phi} \nabla_{\varepsilon} R+\varepsilon_{\delta \varepsilon} \nabla_{\phi} R\right)$ $+G_{\phi \varepsilon \delta}$, thanks to the identity

$$
\begin{aligned}
\frac{1}{2}\left(G_{\phi \delta}^{\alpha} \varepsilon_{\varepsilon \gamma}\right. & \left.+G_{\varepsilon \delta}^{\alpha} \varepsilon_{\phi \gamma}+G_{\phi \gamma}^{\alpha} \varepsilon_{\varepsilon \delta}+G_{\varepsilon \gamma}^{\alpha} \varepsilon_{\phi \delta}\right)=G_{\phi \varepsilon \delta} \delta_{\gamma}^{\alpha}+G_{\phi \varepsilon \gamma} \delta_{\delta}^{\alpha} \\
& -G_{\delta \gamma \phi} \delta_{\varepsilon}^{\alpha}-G_{\delta \gamma \varepsilon} \delta_{\phi}^{\alpha} \cdot \quad \square
\end{aligned}
$$

\section{Four Dimensions}

I. $N=1$ Supersymmetry. Let us take $S O\left(p_{+}, p_{-}\right)=S O(3,1), V=\mathbb{R}^{4}$ with the spinor representation of $\operatorname{Spin}(3,1)=S L(2, \mathbb{C})$. This representation is complexequivalent to the representation on $\mathbb{C}^{2} \oplus \mathbb{C}^{2}$ which is the direct sum of the fundamental representation of $S L(2, \mathbb{C})$ and its complex conjugate. Following the notation of [WB], we will denote the matrix representation of an element $M$ of $\operatorname{sl}(2, \mathbb{C})$ by $(M)_{\alpha}{ }^{\beta} \oplus(M)_{\dot{\alpha}}^{\dot{\beta}}$ with $\operatorname{Tr} M=0$ and $(M)_{\dot{\alpha}}^{\dot{\beta}}=\left((M)_{\alpha}{ }^{\beta}\right)^{*}$. Using the invariant symplectic form $\varepsilon$ on $\mathbb{C}^{2}$ to raise and lower indices, we can consider $M$ as an element of $S^{2}\left(\mathbb{C}^{2}\right)$, i.e. of the form $M_{\alpha \beta}$ with $M_{\alpha \beta}=M_{\beta \alpha}$. We will also identify

$$
\mathscr{H}=\left\{P \in M_{2}(\mathbb{C}): P=-P^{\dagger}\right\}
$$

with the Minkowski 4-space, to write an element of $\mathbb{R}^{4}$ in the form $P_{\alpha \dot{\beta}}$ with $P_{\alpha \dot{\beta}}=-\left(P_{\beta \dot{\alpha}}\right)^{*}$.

Let us first take $\mathscr{S}=\operatorname{End}\left(\mathbb{R}^{4}, V\right)$. The torsion equations become

$$
\begin{aligned}
& d \tau^{\alpha \dot{\beta}}+\tau^{\alpha \dot{\gamma}} \wedge \omega_{\dot{\gamma}}^{\dot{\beta}}+\tau^{\gamma \dot{\beta}} \wedge \omega_{\gamma}{ }^{\alpha}=-\tau^{\alpha} \wedge \tau^{\dot{\beta}},
\end{aligned}
$$

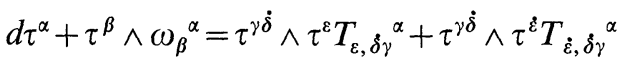

$$
\begin{aligned}
& +\frac{1}{2} \tau^{\gamma \dot{\delta}} \wedge \tau^{\varepsilon \dot{\phi}} T_{\dot{\phi} \varepsilon, \delta \gamma}{ }^{\alpha},
\end{aligned}
$$

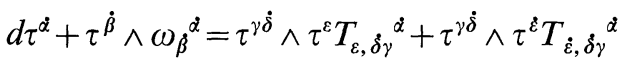

$$
\begin{aligned}
& +\frac{1}{2} \tau^{\gamma \dot{\delta}} \wedge \tau^{\alpha \dot{\phi}} T_{\dot{\phi} \varepsilon, \dot{\gamma} \gamma}{ }^{\alpha},
\end{aligned}
$$

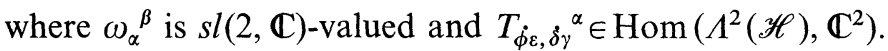

It is well known that the Bianchi identities imply that one can express the torsion and curvature in terms of a complex function $R$, a hermitian tensor $G_{\alpha \dot{\beta}}$ and a complex tensor $W_{\alpha \beta \gamma}$ which is totally symmetric in its indices. We refer to [WB] for the expressions for the components of the torsion tensor, as well as the constraints among $R, G$, and $W$.

There are two $S L(2, \mathbb{C})$-invariant subspaces of $\operatorname{End}\left(\mathbb{R}^{4}, V\right)=\operatorname{End}(\mathscr{H}, V)$, namely

$$
\begin{gathered}
\mathscr{S}_{1}=\left\{M \in \operatorname{End}(\mathscr{H}, V): M_{\alpha \beta}{ }^{\alpha}=0\right\} \quad \text { and } \\
\mathscr{S}_{2}=\left\{M \in \operatorname{End}(\mathscr{H}, V): \exists N_{\dot{\beta}} \text { such that } M_{\alpha \beta}{ }^{\gamma}=\delta_{\alpha}{ }^{\gamma} N_{\dot{\beta}}\right\}
\end{gathered}
$$

Proposition 15. The supergeometry corresponding to $\mathscr{S}_{1}$ has vanishing $R$ and $G$. 
Proof. Using the notation of [WB], the torsions must be writable as

and

$$
T_{\delta, \varepsilon \dot{\varepsilon}}^{\alpha}=\left(M_{\delta}\right)_{\varepsilon \dot{\varepsilon}}^{\alpha}, \quad T_{\delta, \varepsilon \dot{\varepsilon}}^{\alpha}=\left(M_{\delta}\right)_{\varepsilon \dot{\varepsilon}}^{\alpha},
$$

where

$$
T_{\delta \dot{\delta}, \varepsilon \dot{\varepsilon}}^{\alpha}=\left(M_{\delta \dot{\delta}}\right)_{\varepsilon \dot{\varepsilon}}^{\alpha}-\left(M_{\varepsilon \dot{\varepsilon}}\right)_{\delta \dot{\delta}}^{\alpha},
$$

$$
\left(M_{\delta}\right)_{\alpha \dot{\varepsilon}}^{\alpha}=0, \quad\left(M_{\delta}\right)_{\alpha \dot{\varepsilon}}^{\alpha}=0 \text { and }\left(M_{\delta \dot{\delta}}\right)_{\alpha \dot{\varepsilon}}^{\alpha}=0 .
$$

The condition $T_{\delta, \alpha \dot{\varepsilon}}{ }^{\alpha}=0$ gives $G=0$. The condition $T_{\delta, \alpha \dot{\varepsilon}}{ }^{\alpha}=0$ gives $R=0$. If we take $\left(M_{\delta \dot{\delta}}\right)_{\varepsilon \dot{\varepsilon}}^{\alpha}$ to be $-\varepsilon_{\dot{\delta} \dot{\varepsilon}} W_{\delta \varepsilon}^{\alpha}$ then

$$
T_{\delta \dot{\delta}, \varepsilon \dot{\varepsilon}}^{\alpha}=\left(M_{\delta \dot{\delta}}\right)_{\varepsilon \dot{\varepsilon}}^{\alpha}-\left(M_{\varepsilon \dot{\varepsilon}}\right)_{\delta \delta}^{\alpha} \text { and }\left(M_{\delta \dot{\delta}}\right)_{\alpha \dot{\varepsilon}}^{\alpha}=0 \text {. }
$$

Proposition 16. The supergeometry corresponding to $\mathscr{S}_{2}$ has vanishing $G$ and $W$ and constant $R$.

Proof. The torsions must satisfy

and

$$
\begin{aligned}
T_{\dot{\delta}, \varepsilon \dot{\varepsilon}}{ }^{\alpha} & =N_{\delta, \dot{\varepsilon}} \delta_{\varepsilon}{ }^{\alpha}, \\
T_{\delta, \varepsilon \dot{\varepsilon}}{ }^{\alpha} & =N_{\delta, \dot{\varepsilon}} \delta_{\varepsilon}{ }^{\alpha}
\end{aligned}
$$

for some $N_{\dot{\delta}, \dot{\varepsilon}}, N_{\delta, \dot{\varepsilon}}$ and $N_{\delta \dot{\delta}, \dot{\varepsilon}}$. Equation (186) is always satified. Equation (186') implies that

$$
\varepsilon_{\varepsilon \alpha} G_{\delta \dot{\varepsilon}}-3 \varepsilon_{\delta \alpha} G_{\varepsilon \dot{\varepsilon}}-3 \varepsilon_{\delta \varepsilon} G_{\alpha \dot{\varepsilon}}=\varepsilon_{\varepsilon \alpha} H_{\delta \dot{\varepsilon}}
$$

for some $H$. Contracting with $\varepsilon^{\alpha \varepsilon}$ gives $H_{\delta \dot{\varepsilon}}=G_{\delta \dot{\varepsilon}}$ and so $\varepsilon_{\delta \alpha} G_{\varepsilon \dot{\varepsilon}}+\varepsilon_{\delta \varepsilon} G_{\alpha \dot{\varepsilon}}=0$. Contracting with $\varepsilon^{\delta \alpha}$ gives $G=0$. Equation (187) gives

$$
-2 \varepsilon_{\delta \dot{\varepsilon}} W_{\delta \varepsilon}^{\alpha}=N_{\delta \delta, \dot{\varepsilon}} \delta_{\varepsilon}^{\alpha}-N_{\varepsilon \dot{\varepsilon}, \delta} \delta_{\delta}^{\alpha}
$$

Contracting with $\delta_{\alpha}{ }^{\varepsilon}$ gives $N=0$, so $W=0$. The constraints on $R, G$ and $W$ give that $\nabla_{\alpha} R=\nabla_{\dot{\alpha}} R=0$, which implies that $\nabla_{\alpha \dot{\beta}} R=0$.

II. $N=2$ Supersymmetry. Let us take $S O\left(p_{+}, p_{-}\right)=S O(4), V=\mathbb{R}^{8}$ with the spinor representation of $\operatorname{Spin}(4)=S U(2) \times S U(2)$ and $K=\mathbb{H}^{*}=\mathbb{R}^{+} \times S U(2)$. Thinking of $V$ as $\mathbb{H}^{2}$, the representation of Spin (4) $\times K$ on $V$ is given by

$$
\varrho\left(g, g^{\prime}, k\right)\left(v_{1}, v_{2}\right)=\left(g v_{1} k^{-1}, k v_{2}\left(g^{\prime}\right)^{-1}\right)
$$

where $g$ and $g^{\prime}$ are unit quaternions, $k \in \mathbb{H}^{*}$ and $\left(v_{1}, v_{2}\right) \in \mathbb{H}^{2}$. The action of $\operatorname{Spin}(4) \times K$ on $\mathbb{R}^{4}=\mathbb{H}$ is given by $v \rightarrow g v\left(g^{\prime}\right)^{-1}$.It is convenient to use the identification

$$
\mathbb{H}=\left\{P \in M_{2}(\mathbb{C}): \bar{P}=-\varepsilon P \varepsilon\right\},
$$

where $\varepsilon$ is a symplectic form on $\mathbb{C}^{2}$. Using this identification, we will write an element $M$ of $s u(2) \oplus s u(2) \oplus k$ as $(M)_{\alpha}{ }^{\beta} \oplus(M)_{\dot{\alpha}}{ }^{\dot{\beta}} \oplus(M)_{i}{ }^{j}$ with $(M)_{\alpha}{ }^{\alpha}=(M)_{\dot{\alpha}}{ }^{\alpha}=0$. Similarly, we will write an element $P$ of $\mathbb{R}^{4}$ as $P_{\alpha}^{\dot{\beta}}$ and an element $Q$ of $V$ as $(Q)_{\alpha}{ }^{i} \oplus\left(Q^{\prime}\right)_{i}^{\dot{\beta}}$. We will use the symplectic form $\varepsilon$ to raise and lower indices. 
Let us first take $\mathscr{S}=\operatorname{End}\left(\mathbb{R}^{4}, V\right)$. The torsion equations become

$$
\begin{aligned}
& d \tau_{\alpha}^{\dot{\beta}}+\tau_{\alpha}{ }^{\gamma} \wedge \omega_{\dot{\gamma}}^{\dot{\beta}}+\omega_{\alpha}{ }^{\gamma} \wedge \tau_{\gamma}^{\dot{\beta}}=-\tau_{\alpha}{ }^{i} \wedge \tau_{i}^{\prime \dot{\beta}}, \\
& d \tau_{\alpha}{ }^{i}+\omega_{\alpha}{ }^{\beta} \wedge \tau_{\beta}{ }^{i}+\tau_{\alpha}{ }^{j} \wedge \omega_{j}{ }^{i}=\tau_{\gamma}{ }^{\dot{\delta}} \wedge \tau_{\beta}{ }^{j} T_{j}^{\beta}, \delta^{\gamma}{ }_{\alpha}{ }^{i}+\tau_{\gamma}{ }^{\dot{\delta}} \\
& \wedge \tau^{\prime}{ }_{j}^{\dot{\beta}} T_{\dot{\beta}}{ }^{j}, \delta^{\gamma}{ }_{\alpha}{ }^{i}+\frac{1}{2} \tau_{\gamma}^{\dot{\delta}} \wedge \tau_{\varepsilon}^{\dot{\phi}} T_{\dot{\phi}^{\varepsilon}, \delta^{\gamma}{ }_{\alpha}{ }^{i},}, \\
& d \tau^{\prime}{ }_{i}^{\alpha}+\tau^{\prime}{ }_{i}{ }^{\dot{\beta}} \wedge \omega_{\beta}{ }^{\dot{\alpha}}+\omega_{i}{ }^{j} \wedge \tau^{\prime}{ }_{j}^{\dot{\alpha}}=\tau_{\gamma}{ }^{\dot{\delta}} \wedge \tau_{\beta}{ }^{j} T_{j}{ }^{\beta}, \delta^{\gamma}{ }_{i}{ }^{\alpha}+\tau_{\gamma}{ }^{\dot{\delta}}
\end{aligned}
$$

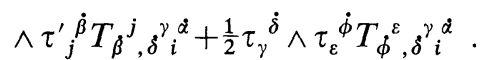

The solution to the Bianchi identities is given in the Minkowski case in [Ho2]. In our case a tedious calculation shows that the solution is given by superfields $U^{\alpha \beta}$, $U^{\prime}{ }_{\alpha \dot{\beta}}, T_{i j}, T^{\prime i j}, W_{\dot{\alpha}}^{\dot{\beta}}, W_{\alpha}^{\prime \beta}, K_{i \dot{\alpha}}^{j}{ }^{\beta}$ and $L_{i \dot{\alpha}}^{j}{ }^{\beta}$. Here $U, U^{\prime}, T$ and $T^{\prime}$ are symmetric and $W$ and $W^{\prime}$ are traceless. The fields satisfy the Hermiticity conditions

$$
\begin{aligned}
& \left(U^{\alpha \beta}\right)^{*}=U_{\alpha \beta},\left(U_{\dot{\alpha} \dot{\beta}}^{\prime}\right)^{*}=U^{\prime \dot{\alpha} \dot{\beta}},\left(T_{i j}\right)^{*}=T^{i j},\left(T^{i j}\right)^{*}=T_{i j}^{\prime}, \\
& \left(W_{\dot{\alpha}}^{\dot{\beta}}\right)^{*}=-W_{\beta}^{\alpha},\left(W_{\alpha}^{\prime \beta}\right)^{*}=-W_{\beta}^{\prime \alpha},\left(K_{i \dot{\alpha}}^{j \beta}\right)^{*}=K_{j \beta}^{i \alpha} \text { and } \\
& \left(L_{i \dot{\alpha}}^{j}\right)^{*}=L_{j \beta}^{i{ }_{j}^{\alpha}} .
\end{aligned}
$$

One finds

$$
\begin{aligned}
& T_{j}^{\beta}, \delta^{\gamma}{ }_{\alpha}^{i}=-\left[K_{j \delta}^{i} \delta^{\beta} \delta_{\alpha}^{\gamma}+L_{j \delta^{i}}^{\gamma} \delta_{\alpha}^{\beta}\right],
\end{aligned}
$$

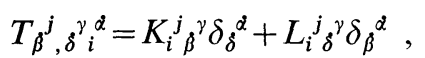

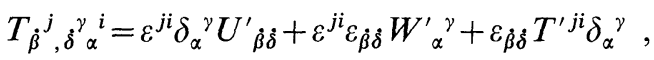

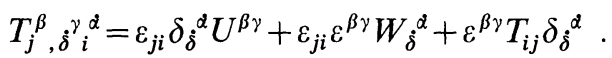

By the freedom in $\operatorname{Im} \delta$, we may assume that $L$ vanishes.

For future use, we will give the curvatures modulo $\tau_{\alpha}^{\dot{\beta}}$. They are

$$
\begin{aligned}
& \Omega_{\alpha}{ }^{\beta}=-\tau_{\alpha}{ }^{i} \wedge \tau_{\varepsilon}{ }^{j}\left(\varepsilon_{j i} U^{\varepsilon \beta}+\varepsilon^{\varepsilon \beta} T_{i j}\right)+\frac{1}{2} \tau_{\varepsilon}{ }^{i} \wedge \tau^{\prime}{ }_{j}{ }^{\dot{\beta}} K_{i}{ }^{j},{ }_{\dot{\beta}}{ }^{\varepsilon} \delta_{\alpha}{ }^{\beta} \\
& -\tau_{\alpha}{ }^{i} \wedge \tau^{\prime}{ }_{j}{ }^{\dot{\beta}} K_{i}{ }^{j},{ }_{\dot{\beta}}{ }^{\beta}-\frac{1}{2} \tau^{\prime}{ }_{j}{ }^{\varepsilon} \wedge \tau^{\prime}{ }_{i}{ }^{\dot{\beta}} \varepsilon^{j i} \varepsilon_{\dot{\varepsilon} \beta} W_{\alpha}{ }^{\beta}, \\
& \Omega_{\dot{\alpha}}^{\dot{\beta}}=\frac{1}{2} \tau_{\alpha}{ }^{i} \wedge \tau_{\varepsilon}{ }_{\varepsilon} \varepsilon_{j i} \varepsilon^{\varepsilon \alpha} W_{\dot{\alpha}}^{\dot{\beta}}-\tau_{\alpha}{ }^{i} \wedge \tau^{\prime}{ }_{j}^{\dot{\beta}} K_{i}{ }^{j},{ }_{\alpha}^{\alpha}+\frac{1}{2} \tau_{\alpha}{ }^{i} \\
& \wedge \tau^{\prime}{ }_{j}^{\varepsilon} K_{i}^{j},{ }_{\dot{\varepsilon}}^{\alpha} \delta_{\dot{\alpha}}^{\dot{\beta}}+\tau^{\prime}{ }_{j}^{\dot{\varepsilon}} \wedge \tau^{\prime}{ }_{i}^{\dot{\beta}}\left(\varepsilon^{j i} U^{\prime}{ }_{\dot{\varepsilon} \dot{\alpha}}+\varepsilon_{\dot{\varepsilon} \dot{\alpha}} T^{\prime i j}\right) \text {, } \\
& \Omega_{i}{ }^{j}=-\tau_{\gamma}{ }^{j} \wedge \tau_{\beta}{ }^{k}\left(\varepsilon_{k i} U^{\beta \gamma}+\varepsilon^{\beta \gamma} T_{i k}\right)-\tau^{\prime}{ }_{i}{ }^{\beta} \wedge \tau_{\alpha}{ }^{k} K_{k}{ }^{j},{ }_{\dot{\beta}}{ }^{\alpha} \\
& +\frac{1}{2} \tau_{\alpha}{ }^{k} \wedge \tau^{\prime}{ }_{j}{ }^{\varepsilon} K_{k}{ }^{j},{ }_{\dot{\varepsilon}}{ }^{\alpha} \delta_{i}{ }^{j}-\tau_{\gamma}{ }^{k} \wedge \tau^{\prime}{ }_{k}{ }^{\dot{\delta}} L_{i}{ }^{j}, \delta^{\gamma}-\tau_{\gamma}{ }^{j} \\
& \wedge \tau^{\prime}{ }_{k}^{\dot{\beta}} K_{i}^{k},{ }_{\beta}{ }^{\gamma}+\tau_{i}^{\prime}{ }_{i}^{\dot{\beta}} \wedge \tau^{\prime}{ }_{k}{ }^{\varepsilon}\left(\varepsilon^{k j} U^{\prime}{ }_{\dot{\varepsilon} \beta}+\varepsilon_{\dot{\varepsilon} \beta} T^{\prime j k}\right) \text {. }
\end{aligned}
$$

The superfields will have to satisfy further first order differential equalities, which we will not bother to derive here (see Sect. VIII).

\section{E. Six Dimensions}

Let us take $S O\left(p_{+}, p_{-}\right)=S O(5,1), V=\mathbb{R}^{8}$ with the spinor representation of $\operatorname{Spin}(5,1)=S L(2, \mathbb{H})$. This representation is the fundamental representation of $S L(2, \mathbb{H})$ on $\mathbb{H}^{2}$. Following $[K T]$, it turns out to be most convenient to use another 
equivalent form of $\operatorname{Spin}(5,1)$, which is $S U^{*}(4)$. Let $J$ be a real skew-symmetric operator on $\mathbb{C}^{4}, J^{2}=-1$, and let $\varepsilon$ be a real skew-symmetric operator on $\mathbb{C}^{2}$, $\varepsilon^{2}=-1$. Then

Put

$$
S U^{*}(4)=\{A \in S L(4, \mathbb{C}): J \bar{A}=A J\} \text {. }
$$

$$
V=\{v \in M(4,2, \mathbb{C}): \bar{v}=J v \varepsilon\} .
$$

Then $S U^{*}(4)$ acts on $V$ by $\varrho(A) v=A v$. We can identify $\mathbb{R}^{6}$ with

$$
\mathscr{H}=\left\{B \in M(4, \mathbb{C}): J \bar{B}=-B J, B^{T}=-B\right\},
$$

with the $S U^{*}(4)$ action given by $B \rightarrow A B A^{T}$. There is a map from $S^{2}(V)$ to $\mathscr{H}$ given by $\left(v, v^{\prime}\right) \rightarrow v \varepsilon v^{\prime T}+v^{\prime} \varepsilon v^{T}$, which gives a super Euclidean algebra on $\mathbb{R}^{6} \mid V$.

Let us denote the components of $A \in s u^{*}(4)$ by $A_{\beta}{ }^{\alpha}, B \in M(4, \mathbb{C})$ by $B^{\alpha \beta}$ and $v \in M(4,2, \mathbb{C})$ by $v_{i}^{\alpha}$.

Let us first take $\mathscr{S}=\operatorname{End}\left(\mathbb{R}^{6}, V\right)$. The torsion equations become

$$
\begin{gathered}
d \tau^{\alpha \beta}+\tau^{\alpha \gamma} \wedge \omega_{\gamma}{ }^{\beta}+\tau^{\gamma \beta} \wedge \omega_{\gamma}{ }^{\alpha}=-\varepsilon^{i j} \tau_{i}^{\alpha} \wedge \tau_{j}^{\beta}, \\
d \tau_{i}^{\alpha}+\tau_{i}^{\beta} \wedge \omega_{\beta}{ }^{\alpha}=\tau^{\gamma \delta} \wedge \tau_{j}^{\varepsilon} T_{\varepsilon, \delta \gamma i}^{j}+\frac{1}{2} \tau^{\gamma \delta} \wedge \tau^{\varepsilon \phi} T_{\phi \varepsilon, \delta \gamma i}{ }^{\alpha} .
\end{gathered}
$$

The solution for the Bianchi identities is given in terms of a tensor $M^{\beta \gamma}$ which is symmetric in $\beta \gamma$ and satisfies $\nabla_{\beta}^{i} M^{\beta \gamma}=0$ [ST]. Explicitly,

$$
\begin{gathered}
T_{\varepsilon, \delta \gamma i}^{j} \alpha=\varepsilon_{\varepsilon \delta \gamma \beta} M^{\beta \alpha} \delta_{i}{ }^{j}, \\
T_{\phi \varepsilon, \delta \gamma i}^{\alpha}=\varepsilon_{i j}\left[\varepsilon_{\phi \varepsilon \delta \sigma} \nabla_{\gamma}^{j} M^{\sigma \alpha}-\varepsilon_{\phi \varepsilon \gamma \sigma} \nabla_{\delta}^{j} M^{\sigma \alpha}-\varepsilon_{\delta \gamma \phi \sigma} \nabla_{\varepsilon}^{j} M^{\sigma \alpha}+\varepsilon_{\delta \gamma \varepsilon \sigma} \nabla_{\phi}^{j} M^{\sigma \alpha}\right] .
\end{gathered}
$$

There are two $S U^{*}(4)$-invariant subspaces of $\operatorname{End}\left(\mathbb{R}^{6}, V\right)=\operatorname{End}(\mathscr{H}, V)$, namely

$$
\begin{gathered}
\mathscr{I}_{1}=\left\{M \in \operatorname{End}(\mathscr{H}, V): M_{\alpha \beta i}{ }^{\beta}=0\right\} \text { and } \\
\mathscr{S}_{2}=\left\{M \in \operatorname{End}(\mathscr{H}, V): \exists N_{\beta i} \text { such that } M_{\alpha \beta i}{ }^{\gamma}=\delta_{\alpha}{ }^{\gamma} N_{\beta i}-\delta_{\beta}{ }^{\gamma} N_{\alpha i}\right\} .
\end{gathered}
$$

Clearly the geometry corresponding to $\mathscr{S}_{1}$ is the same as that corresponding to $\mathscr{S}$. On the other hand, the geometry corresponding to $\mathscr{S}_{2}$ is easily seen to have vanishing $M$, and so is flat.

\section{Higher Order Obstructions to Integrability}

The torsion tensor of a $G$-structure gives a first-order obstruction to the flatness of the $G$-structure. There are also higher order obstructions, which are given by the Spencer homology groups. An elegant exposition of this theory is given in [Gu]. We will briefly review $[\mathrm{Gu}]$ in order to fix notation. (We will consider everything to be $\mathbb{Z}_{2}$ graded, without writing so explicitly.)

Given the Lie algebra $g \subset g l(W)$ of $G$, define $g^{(1)} \subset \operatorname{End}(W, g)$ as in Sect. IV. Define $g^{(k)}$ inductively by

$$
g^{(k)}=\left\{S \in \operatorname{End}\left(W, g^{(k-1)}\right): S(w)\left(w^{\prime}\right)=S\left(w^{\prime}\right)(w) \text { for all } w, w^{\prime} \in W\right\} .
$$

There is a Lie group $\mathscr{G}^{k}$ whose Lie algebra is $g \oplus g^{(1)} \oplus \ldots \oplus g^{(k)}$, with an appropriately defined bracket. Given a $G$-structure which is $k^{\text {th }}$ order flat, one can 
define a principal bundle $E^{k}$ over $M$ with structure group $\mathscr{G}^{k}$. There is a canonically defined 1-form $\Gamma^{k}$ on $E^{k}$ with value in $V \oplus g \oplus \ldots \oplus g^{(k-1)}$. We will denote its homogeneous parts by $\tau, \omega^{0}, \ldots, \omega^{k-1}$.

(In the special case $k=0$, one has that $\mathscr{G}^{0}=G, E^{0}$ is the usual frame bundle and $\Gamma^{0}$ is the canonical 1 -form $\tau$. The case $k>0$ is a generalization of this.)

Let us define

$$
\begin{gathered}
T=d \tau+\frac{1}{2}\left(\left[\tau, \omega^{0}\right]+\left[\omega^{0}, \tau\right]\right) \\
\Omega^{i}=d \omega^{i}+\frac{1}{2}\left(\left[\tau, \omega^{i+1}\right]+\left[\omega^{0}, \omega^{i}\right]+\ldots+\left[\omega^{i+1}, \tau\right]\right), \quad 0<i<k-1 .
\end{gathered}
$$

One can verify that for $i<k-1, d \omega^{i}$ is canonically given on $E^{k}$, and so $\Omega^{i}$ has the corresponding flat space value. On the other hand, $d \omega^{k-1}$ is not canonically given if the $G$-structure is not $(k+1)^{\text {th }}$ order flat. Let us choose a $\mathscr{G}^{k}$-equivariant horizontal $g^{(k)}$-valued 1 -form $\omega^{k}$ and define

$$
\Omega^{k-1}=d \omega^{k-1}+\frac{1}{2}\left(\left[\tau, \omega^{k}\right]+\left[\omega^{0}, \omega^{k-1}\right]+\ldots+\left[\omega^{k}, \tau\right]\right) .
$$

The exterior differentiation of

gives

$$
\Omega^{k-2}=d \omega^{k-2}+\frac{1}{2}\left(\left[\tau, \omega^{k-1}\right]+\ldots+\left[\omega^{k-1}, \tau\right]\right)
$$

$$
\nabla \Omega^{k-2}=\left[\tau, \Omega^{k-1}\right],
$$

where $\nabla \Omega^{k-2}=d \Omega^{k-2} \circ H$ denotes the covariant exterior derivative. As $\Omega^{k-2}$ is canonically given, if $\nabla \Omega^{k-2}$ vanishes in the flat geometry then $\left[\tau, \Omega^{k-1}\right]$ vanishes on $E^{k}$.

Let us define the chain group

$$
C^{k, l}=g^{(k-1)} \otimes \Lambda^{l}\left(W^{*}\right) .
$$

There is a boundary map

$$
\delta: C^{k, l} \rightarrow C^{k-1, l+1} \quad[\mathrm{Gu}] .
$$

Then given a choice of $\omega^{k}$, the condition $\left[\tau, \Omega^{k-1}\right]=0$ implies $\Omega^{k-1} \in Z^{k, 2}$. The freedom of choosing $\omega^{k}$ means that there is a well-defined element of $H^{k, 2}$ which gives an obstruction to the $(k+1)^{\text {th }}$ order flatness of the $G$-structure. The group $H^{k, 2}$ is the Spencer homology group [Sp, Gu].

Let us consider the setup of Sect. IV with unextended supersymmetry. That is, the structure group $G$ is $\operatorname{Spin}\left(p_{+}, p_{-}\right) \tilde{x} \mathscr{P}$, where $\mathscr{I}$ is a $\operatorname{Spin}\left(p_{+}, p_{-}\right)$-invariant subspace of End $\left(\mathbb{R}^{p}, V\right)$.

Proposition 17. If $k>0$ then $g^{(k)}=\left(\mathscr{P} \otimes S^{k}\left(\left(\mathbb{R}^{p}\right)^{*}\right)\right) \cap\left(V \otimes S^{k+1}\left(\left(\mathbb{R}^{p}\right)^{*}\right)\right)$.

Proof. In components, an element $M$ of $g^{(k)}$ can be written as a tensor $M_{A_{1} \ldots A_{k+1}{ }_{B}}$ which is graded symmetric in $\left(A_{1}, \ldots, A_{k+1}\right)$ and is such $\left(M_{A_{1} \ldots A_{k}}\right)_{A_{k+1}}$ $=M_{A_{1} \ldots A_{k+1}}{ }^{B}$ denotes an element $M_{A_{1} \ldots A_{k}}$ of $g$. First suppose that $B$ is an even index. For $\left(M_{A_{1} \ldots A_{k}}\right)_{A_{k+1}}{ }^{B}$ to be nonzero, $A_{k+1}$ must also be an even index. By the symmetry in the lower indices, for $\left(M_{A_{1} \ldots A_{k}}\right)_{A_{k+1}}{ }^{B}$ to be nonzero, each $A_{i}$ must be an even index. Then we are reduced to the case of $s o\left(p_{+}, p_{-}\right)^{(k)}$, which is known to be zero if $k>0$. 
Now suppose that $B$ is an odd index. If $A_{k+1}$ is an odd index then

$$
M_{A_{1} \ldots A_{k+1}}{ }^{B}=\left(M_{A_{1} \ldots A_{k}}\right)_{A_{k+1}}{ }^{B}=\frac{1}{2}\left(M_{A_{1} \ldots A_{k}}\right)_{a}^{b}\left(\sigma_{b}{ }^{a}\right)_{A_{k+1}}{ }^{B}
$$

vanishes. By the symmetry of the lower indices, for $M_{A_{1} \ldots A_{k+1}}{ }^{B}$ to be nonzero, each $A_{i}$ must be an even index.

In components, an element of $C^{k, l}$ has the form $M_{C_{1} \ldots C_{l}, A_{1} \ldots A_{k}}{ }^{B}$, where $M$ is graded skew symmetric in the $C$ indices and graded symmetric in the $A$ indices. The boundary map $\delta: C^{k, l} \rightarrow C^{k-1, l+1}$ is given by

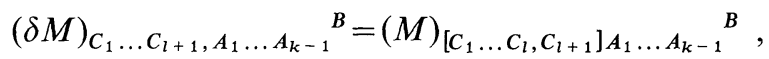

where [] denotes antisymmetrization.

The group $H^{0,2}$ gives the torsion obstruction which we have already discussed.

Proposition 18. If $\mathscr{S}=\operatorname{End}\left(\mathbb{R}^{p}, V\right)$ then $H^{k, 2}=0$ for $k>1$.

Proof. $H^{k, 2}$ is the middle homology of the sequence

$$
\ldots \rightarrow g^{k} \otimes \Lambda^{1}\left(W^{*}\right) \rightarrow g^{k-1} \otimes \Lambda^{2}\left(W^{*}\right) \rightarrow g^{k-2} \otimes \Lambda^{3}\left(W^{*}\right) \rightarrow \ldots .
$$

If $k>1$ then by Proposition $17, g^{k-1}=V \otimes S^{k}\left(\left(\mathbb{R}^{p}\right)^{*}\right)$. Suppose that $M_{C_{1} C_{2}, A_{1} \ldots A_{k}{ }^{B}}$ denotes an element $M$ of $g^{k-1} \otimes \Lambda^{2}\left(W^{*}\right)$ with $\delta M=0$. If both $C$ indices are odd then

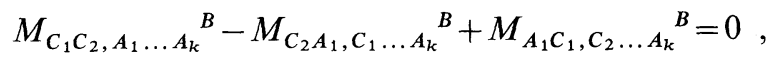

which implies that $M_{C_{1} C_{2}, A_{1} \ldots A_{k}{ }^{B}}$ vanishes. If $C_{1}$ is odd and $C_{2}$ is even then

$$
M_{C_{1} C_{2}, A_{1} \ldots A_{k}}^{B}+M_{C_{2} A_{1}, C_{1} \ldots A_{k}}^{B}+M_{A_{1} C_{1}, C_{2} \ldots A_{k}}{ }^{B}=0 \text {, }
$$

which implies

$$
M_{C_{1} C_{2}, A_{1} \ldots A_{k}}{ }^{B}=M_{C_{1} A_{1}, C_{2} \ldots A_{k}}{ }^{B} .
$$

Then $M=\delta N$, where $N \in g^{k} \otimes \Lambda^{1}\left(W^{*}\right)$ is given by

$$
N_{C_{1}, A_{1} \ldots A_{k+1}}{ }^{B}=M_{C_{1} A_{1}, A_{2} \ldots A_{k+1}{ }^{B}} \text {. }
$$

If $C_{1}$ and $C_{2}$ are both even then

$$
M_{C_{1} C_{2}, A_{1} \ldots A_{k}}{ }^{B}+M_{C_{2} A_{1}, C_{1} \ldots A_{k}}{ }^{B}+M_{A_{1} C_{1}, C_{2} \ldots A_{k}}{ }^{B}=0 .
$$

In this case one can show that $M \in \operatorname{Im} \delta$ by the same agument as is used to show that the Spencer homology vanishes for the group $G L(n, \mathbb{R})$.

Proposition 19. If $\mathscr{S}=$ End $\left(\mathbb{R}^{p}, V\right)$ then the homology group $H^{1,2}$ is isomorphic to that of a usual $S O\left(p_{+}, p_{-}\right)$Riemannian geometry.

Proof. The group $H^{1,2}$ is the middle homology of the sequence

$$
\ldots \rightarrow g^{1} \otimes \Lambda^{1}\left(W^{*}\right) \rightarrow g \otimes \Lambda^{2 *}(W) \rightarrow W \otimes \Lambda^{3}\left(W^{*}\right) \rightarrow \ldots .
$$


If $M \in g \otimes \Lambda^{2}\left(W^{*}\right)$ then the component equations of $\delta M=0$ become

$$
\begin{aligned}
& M_{E_{1} E_{2}, E_{3}}{ }^{O_{1}}+M_{E_{2} E_{3}, E_{1}}{ }^{O_{1}}+M_{E_{3} E_{1}, E_{2}}{ }^{O_{1}}=0, \\
& M_{E_{1} E_{2}, O_{1}}{ }^{o_{2}}-M_{O_{1} E_{2}, E_{1}}^{o_{2}}+M_{O_{1} E_{1}, E_{2}}^{o_{2}}=0 \text { ， } \\
& M_{O_{1} O_{2}, E_{1}}^{O_{3}}+M_{E_{1} O_{1}, O_{2}}{ }^{O_{3}}+M_{E_{1} O_{2}, o_{1}}^{O_{3}}=0, \\
& M_{O_{1} O_{2}, O_{3}}{ }^{o_{4}}+M_{O_{2} O_{3}, O_{1}}{ }^{o_{4}}+M_{O_{3} O_{1}, o_{2}}^{o_{4}}=0,
\end{aligned}
$$

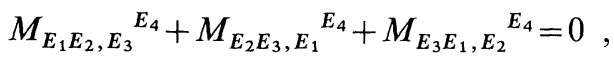

$$
\begin{aligned}
& M_{O_{1} E_{2}, E_{1}}{ }^{E_{3}}-M_{O_{1} E_{1}, E_{2}}{ }^{E_{3}}=0 \text {, } \\
& M_{O_{1} O_{2}, E_{1}}{ }^{E_{2}}=0
\end{aligned}
$$

where $E$ or $O$ denotes an even or odd index.

Note that because of the spinor representation of $S O\left(p_{+}, p_{-}\right)$, Eq. (230) is related to Eq. (233), Eq. (231) is related to Eq. (234) and Eq. (232) is related to Eq. (235). Because $\mathscr{S}=$ End $\left(\mathbb{R}^{p}, V\right)$, as in the proof of Proposition 18, Eq. (229) gives no contribution to $H^{1,2}$. Equation (235) implies that Eq. (232) has no content. Equation (233) gives the same contribution to $H^{1,2}$ as in ordinary Riemannian geometry, that is, a tensor with the symmetries of the Riemannian curvature tensor of an $S O\left(p_{+}, p_{-}\right)$geometry. Equation (230) implies that there is a tensor $P$ such that

$$
M_{O_{1} E_{1}, E_{2}}^{O_{2}}=-\frac{1}{4} M_{E_{1} E_{2}, E_{3}}^{E_{4}}\left({\sigma_{E_{4}}}^{E_{3}}\right)_{O_{1}}^{O_{2}}+P_{O_{1} E_{1}, E_{2}}^{O_{2}}
$$

where $P$ is symmetric in $E_{1}$ and $E_{2}$. Then $P$ lies in $\operatorname{Im} \delta$, and so Eq. (230) gives no new contribution to $H^{1,2}$. Because $s o\left(p_{+}, p_{-}\right)^{(1)}$ vanishes, Eq. (234) implies $M_{O_{1} E_{1}, E_{2}}{ }^{E_{3}}=0$. Then Eq. (231) becomes $M_{O_{1} O_{2}, E_{1}}^{O_{3}}=0$.

Propostion 19 shows that flatness of a superRiemannian structure with no internal symmetry group is given by flatness of the reduced space, i.e. there is no new curvature in the odd directions.

Let us now consider the case when $G=\operatorname{Spin}\left(p_{+}, p_{-}\right)$and $W=\mathbb{R}^{p} \oplus V$. We know that when the hypotheses of Proposition 4 are satisfied, first order flatness implies second order flatness. Let us show more generally that there are no formal obstructions to flatness other than the torsion.

Proposition 20. For $G=\operatorname{Spin}\left(p_{+}, p_{-}\right)$, acting faithfully on $V$, we have $g^{(k)}=0$ for $k>0$.

Proof. This follows from the proof of Proposition 17.

Proposition 21. For $G=\operatorname{Spin}\left(p_{+}, p_{-}\right)$, acting faithfully on $V$, we have $H^{k, 2}=0$ for $k>0$.

Proof. Because $g^{(k)}$ vanishes for $k>0, H^{k, 2}$ automatically vanishes for $k>1$. If $\delta: g \otimes \Lambda^{2}\left(W^{*}\right) \rightarrow W \otimes \Lambda^{3}\left(W^{*}\right)$ is the boundary map then $H^{1,2}=\operatorname{Ker} \delta$. Written in 
components, this becomes

$$
\begin{aligned}
& M_{E_{1} E_{2}, O_{1}}{ }^{O_{2}}=0, \\
& M_{E_{1} O_{1}, O_{2}}{ }^{O_{3}}+M_{E_{1} O_{2}, O_{1}}{ }^{O_{3}}=0, \\
& M_{O_{1} O_{2}, O_{3}}{ }^{O_{4}}+M_{O_{2} O_{3}, O_{1}}{ }^{O_{4}}+M_{O_{3} O_{1}, O_{2}}{ }^{o_{4}}=0, \\
& M_{E_{1} E_{2}, E_{3}}{ }^{E_{4}}+M_{E_{2} E_{3}, E_{1}}{ }^{E_{4}}+M_{E_{3} E_{1}, E_{2}}{ }^{E_{4}}=0, \\
& M_{O_{1} E_{2}, E_{1}}{ }^{E_{3}}-M_{O_{1} E_{1}, E_{2}}{ }^{E_{3}}=0, \\
& M_{O_{1} O_{2}, E_{1}}{ }^{E_{2}}=0,
\end{aligned}
$$

where $E$ or $O$ denotes an even or odd index.

Equation (237) implies that Eq. (240) has no content, and Eq. (242) implies that Eq. (239) has no content. Because $s o\left(p_{+}, p_{-}\right)^{(1)}=0$, Eq. (241) implies $M_{O_{1} E_{1}, 2} E_{3}=0$. Then Eq. (238) has no content.

\section{Superconformal Geometry}

The superconformal Lie algebras tend to be simple super Lie algebras (a classification of which is given in [Ka]). They have a filtration

where

$$
g=g^{(-1)} \oplus g^{(-1 / 2)} \oplus g^{(0)} \oplus g^{(1 / 2)} \oplus g^{(1)}
$$

$$
\begin{aligned}
& g^{(-1)}=g^{(1)}=\mathbb{R}^{p}, \\
& g^{(-1 / 2)}=g^{(1 / 2)}=V
\end{aligned}
$$

and

$$
g^{(0)}=\operatorname{so}\left(p_{+}, p_{-}\right) \oplus \mathbb{R} \oplus k
$$

with $k$ being a Lie algebra which represents an additional symmetry. The even part of $g$ is $s o\left(p_{+}+1, p_{-}+1\right) \oplus k$, which is the usual conformal Lie algebra plus $k$. The commutator of $g^{(-1 / 2)}$ and $g^{(1 / 2)}$ is nonzero in $g^{(0)}$ and is given by the model torsion. This is similar to what happens in CR geometry, in which the nonzero torsion corresponds to a nondegenerate Levi form [CM].

We will need the superconformal Lie algebras for the following spacetimes, where $N$ denotes the extended supersymmetry index:

\begin{tabular}{lll}
\hline Signature & Lie algebra & $k$ \\
\hline$(+)$ & $\operatorname{osp}_{\mathbb{R}}(N \mid 1)$ & $s o_{\mathbb{R}}(N)$ \\
$(++)$ & $\operatorname{osp}_{\mathbb{C}}(N \mid 1)$ & $s o_{\mathbb{C}}(N)$ \\
$(++++)$ & $s l_{\mathbb{H}}(2 \mid N)$ if $N \neq 2$ & $g l_{\mathbb{H}}(N)$ \\
& $s l_{\mathbb{H}}(2 \mid 2) / \mathbb{R}$ if $N=2$ & $s l_{\mathbb{H}}(2)$ \\
$(-+)$ & $\operatorname{osp}_{\mathbb{R}}\left(N_{L} \mid 1\right) \oplus \operatorname{spp}_{\mathbb{R}}\left(N_{R} \mid 1\right)$ & $s o_{\mathbb{R}}\left(N_{L}\right) \oplus s o_{\mathbb{R}}\left(N_{R}\right)$ \\
$(-++)$ & $\operatorname{osp}_{\mathbb{R}}(N \mid 2)$ & $s o_{\mathbb{R}}(N)$ \\
$(-+++)$ & $s u(N \mid 2,2)$ if $N \neq 4$ & $u(N)$ \\
& $s u(4 \mid 2,2) / \mathbb{R}$ if $N=4$ & $s u(4)$ \\
$(-+++++)$ & $\operatorname{hosp}(N \mid 2)$ & $h o(N)$ \\
\hline
\end{tabular}


If we consider $g^{(-1)}$ to be the even tangent space and $g^{(-1 / 2)}$ to be the odd tangent space, then the subspace $g^{(0)} \oplus g^{(1 / 2)}$ of $g$ acts on $g^{(-1)} \oplus g^{(-1 / 2)}$ by

$$
\begin{aligned}
& \operatorname{ad} g^{(0)}: g^{(-1)} \rightarrow g^{(-1)}, \quad \text { ad } g^{(0)}: g^{(-1 / 2)} \rightarrow g^{(-1 / 2)} \quad \text { and } \\
& \operatorname{ad} g^{(1 / 2)}: g^{(-1)} \rightarrow g^{(-1 / 2)} .
\end{aligned}
$$

Let $G$ denote a super Lie group with Lie algebra $g$ and let $G_{0}$ denote a subgroup with Lie algebra

$$
g_{0}=g^{(0)} \oplus g^{(1 / 2)} \oplus g^{(1)}
$$

Note because the adjoint action of $G_{0}$ on $g$ preserves $g_{0}$, there is a representation $\varrho$ of $G_{0}$ into End $\left(g / g_{0}\right)$. Let $G^{\prime}$ denote the image $\varrho(G)$.

The model spaces for a superconformal structure will be homogeneous spaces of the form $G / G_{0}$. These will turn out to be Grassmannian manifolds or subspaces thereof on which a quadratic form vanishes. The reduced spaces will be conformal compactifications of the flat Lorentzian (or Euclidean) spaces.

We will consider a superconformal geometry to be given by a Cartan connection [Kob] on a supermanifold $X$. That is to say, we have a $g$-valued connection $\omega$ on a principal $G_{0}$ bundle such that

1. $\omega$ is $G_{0}$ equivariant.

2. For all $X \in g_{0}, \omega\left(V_{X}\right)=X$, where $V_{X}$ is the vertical vector field corresponding to $X$.

3. $\omega$ gives an isomorphism between the tangent space of a point in the bundle and the Lie algebra $g$.

Suppose that we can write $g^{(0)}$ as the direct sum $h \oplus k$ of two Lie subalgebras. [We will take $h$ to be $s o\left(p_{+}, p_{-}\right) \oplus \mathbb{R}$ and $k$ to be the additional symmetry group.] Suppose that we are given the $g^{(-1)}, g^{(-1 / 2)}$ and $h$ parts of a putative Cartan connection, denoted by $P, Q$ and $M$. We will want to be able to find the $k, g^{(1 / 2)}$ and $g^{(1)}$ parts to complete a Cartan connection $\omega$. The 1 -forms $P, Q$ and $M$ will have to satisfy some conditions, namely

$1^{\prime}$. For all $X \in g_{0}, P\left(V_{X}\right)=Q\left(V_{X}\right)=0$ and $M\left(V_{X}\right)=(X)_{h}$.

$2^{\prime} . P \oplus Q \oplus M$ is $G_{0}$ equivariant, where $G_{0}$ acts on $g^{(-1)} \oplus g^{(-1 / 2)} \oplus h$ $=g /\left(k \oplus g^{(1 / 2)} \oplus g^{(1)}\right)$ via the adjoint action on $g$.

3'. A tangent vector $V$ on the principal bundle is vertical if $P(V)=Q(V)=0$.

The idea is to find curvature obstructions to the conformal flatness of a space. To do so, we will find the curvature of some specified Cartan connection $\omega$. In order to specify $\omega$, we will want to put some conditions on its curvature $\Omega$. These conditions should be weak enough so that a superRiemannian geometry also has a superconformal structure.

Given a $G_{0}$ principal bundle, the homomorphism $\varrho$ gives a $G^{\prime}$ principal bundle. $G^{\prime}$ can be written as

$$
G^{\prime}=\left(\operatorname{Spin}\left(p_{+}, p_{-}\right) \times \mathbb{R}^{+} \times K\right) \tilde{\times} \mathscr{S},
$$

where the subspace $\mathscr{S}$ of End $\left(\mathbb{R}^{p}, V\right)$ is given by

$$
\mathscr{S} \cong V^{*}=g^{(1 / 2)} \text {. }
$$

Here the inclusion $V^{*} \subset$ End $\left(\mathbb{R}^{p}, V\right)$ is given by the Clifford multiplication of $\mathbb{R}^{p}$ on $V^{*}$. The principal $G^{\prime}$ bundle coming from a $G_{0}$ bundle will inherit the canonical forms $P$ and $Q$. If we reduce a first order flat superRiemannian structure with 
connection, having $\mathscr{S}=$ End $\left(\mathbb{R}^{p}, V\right)$, to a superRiemannian structure with $\mathscr{S}=V^{*}$, then the induced $\operatorname{Spin}\left(p_{+}, p_{-}\right) \tilde{\times} \mathrm{V}^{*}$ connection will generally have nonzero torsion, which lies in $\operatorname{Hom}\left(\Lambda^{2}\left(\mathbb{R}^{p}\right), V\right) \oplus \operatorname{Hom}\left(\mathbb{R}^{p} \oplus V, V\right)$. Consequently, it would be too stringent to require first order flatness of the $G^{\prime}$ structure over $X$. In order to specify the Cartan connection, we will require that the $g^{(-1)}$ part of $\Omega$ vanishes, that the $g^{(-1 / 2)}$ part of $\Omega$ lies in a certain subspace of $\operatorname{Hom}\left(\Lambda^{2}\left(\mathbb{R}^{p}\right), V\right) \oplus \operatorname{Hom}\left(\mathbb{R}^{p} \oplus V, V\right)$ and that the $g^{(0)}$ part of $\Omega$ satisfy a tracelessness condition analogous to the vanishing of the Ricci part of a curvature tensor. These conditions must be invariant under the action of $G_{0}$. As the super Lie groups change very much with the spacetime dimension, we will have to look at the cases individually.

\section{B. Two Dimensions}

As is well known, conformal flatness is somewhat different in two dimensions as compared to higher dimensions. We will only discuss case II of $(1,1)$ supersymmetry. The other two-dimensional cases are similar. The global superconformal algebra is $\operatorname{osp}_{\mathbb{C}}(1 \mid 1)$. The model space $X$ is a supermanifold arising from a homogeneous $\mathbb{R}^{2}$ vector bundle over $S^{2}$. In the two-dimensional case it would be wrong to construct a Cartan connection with values in $\operatorname{osp}_{\mathbb{C}}(1 \mid 1)$, as the Cartan connection is a local construction, and the local automorphism group $g$ is much larger than osp $\mathrm{C}_{\mathbb{C}}(1 \mid 1)$. In fact, $g$ is a subalgebra of the $\mathbb{Z} \oplus(\mathbb{Z}+1 / 2)$ graded NeveuSchwarz superextension of the Virasoro algebra [NS], with even generators $\left\{L_{m}\right\}_{m=-\infty}^{1}$, odd generators $\left\{G_{r}\right\}_{r=-\infty+1 / 2}^{1 / 2}$ and relations

$$
\begin{aligned}
& {\left[L_{m}, L_{n}\right]=(m-n) L_{m+n},} \\
& {\left[L_{m}, G_{r}\right]=(m / 2-r) G_{m+r},} \\
& \left\{G_{r}, G_{s}\right\}=2 L_{r+s} .
\end{aligned}
$$

There is an induced grading

$$
g=g^{(-1)} \oplus g^{(-1 / 2)} \oplus \ldots
$$

with $g^{(m)}$ generated by $L_{-m}$ and $g^{(m+1 / 2)}$ generated by $G_{-m-1 / 2}$.

A $g$-valued Cartan connection can be written in the form

$$
\omega=\left(\bigoplus_{m=-1}^{\infty} \omega^{(m)}\right) \oplus\left(\bigoplus_{m=-1 / 2}^{\infty+1 / 2} \omega^{(m)}\right),
$$

where $\left\{\omega^{(m)}\right\}_{m=-1}^{\infty}$ are even $\mathbb{C}$-valued 1 -forms and $\left\{\omega^{(m)}\right\}_{m=-1 / 2}^{\infty+1 / 2}$ are odd $\mathbb{C}$-valued 1forms. Let us take $h=\mathbb{C}$ and $k=0$. Here $P$ is $\omega^{(-1)}, Q$ is $\omega^{(-1 / 2)}$ and $M$ is $\omega^{(0)}$.

Proposition 22. Given $P, Q$ and $M$ satisfying $1^{\prime}, 2^{\prime}, 3^{\prime}$ and $\Omega^{P}=0$, there is a Cartan connection $\omega$ extending $P \oplus Q \oplus M$ such that $\omega$ is flat.

Proof. First, let us take any Cartan connection $\omega$ extending $P \oplus Q \oplus M$. Writing the curvature $\Omega$ as

$$
\Omega=\left(\bigoplus_{m=-1}^{\infty} \Omega^{(m)}\right) \oplus\left(\underset{m=-1 / 2}{\infty+1 / 2} \Omega^{(m)}\right)
$$


we find

$$
\begin{gathered}
\Omega^{(m)}=d \omega^{(m)}+\sum_{p+q=m}(-p+q) \omega^{(p)} \wedge \omega^{(q)}+2 \sum_{r+s=m} \omega^{(r)} \wedge \omega^{(s)} \quad \text { for } m \in \mathbb{Z}, \\
d \omega^{(m)}+2 \sum_{p+r=m}(-p / 2+r) \omega^{(p)} \wedge \omega^{(r)} \quad \text { for } m \in \mathbb{Z}+1 / 2 .
\end{gathered}
$$

The Bianchi identity is

$$
\begin{aligned}
d \Omega^{(m)}= & 2 \sum_{p+q=m}(-p+q) \omega^{(p)} \wedge \Omega^{(q)}+4 \sum_{r+s=m} \omega^{(r)} \wedge \Omega^{(s)}, \quad m \in \mathbb{Z} \\
& 2 \sum_{p+r=m}(-p / 2+r)\left(\omega^{(p)} \wedge \Omega^{(r)}-\Omega^{(p)} \wedge \omega^{(r)}\right) \quad \text { for } m \in \mathbb{Z}+1 / 2 .
\end{aligned}
$$

By hypothesis, we have that $\Omega^{(-1)}$ vanishes. For $m \in \mathbb{Z}, m \geqq-1$, we will assume that $\Omega^{(-1)}, \Omega^{(-1 / 2)}, \ldots, \Omega^{(m)}$ vanish and show that it is possible to modify $\omega^{(m+3 / 2)}$ and $\omega^{(m+2)}$ so that $\Omega^{(m+1 / 2)}$ and $\Omega^{(m+1)}$ vanish. By the Bianchi identity, we have

$$
0=2(2+m) \omega^{(-1)} \wedge \Omega^{(m+1)}+4 \omega^{(-1 / 2)} \wedge \Omega^{(m+1 / 2)} .
$$

Thus $\Omega^{(m+1 / 2)}$ must be proportionate to $\omega^{(-1)}$ and so it is possible to modify $\omega^{(m+3 / 2)}$ in order to make $\Omega^{(m+1 / 2)}$ vanish. By the Bianchi identity, we then have

$$
0=2(m+2) \omega^{(-1)} \wedge \Omega^{(m+3 / 2)}+2(m / 2+1) \Omega^{(m+1)} \wedge \omega^{(-1 / 2)} .
$$

Thus $\Omega^{(m+1)}$ must be proportionate to $\omega^{(-1)}$ and so it is possible to modify $\omega^{(m+2)}$ so that $\Omega^{(m+1)}$ vanishes. By induction, we can complete $\omega$ so as to make $\Omega$ vanish.

In order to clarify the question of superconformal structures in two dimensions, suppose that $X$ is a real supermanifold of dimension $2 \mid 2$ and that the frame bundle of $X$ has a reduction to a subbundle with the structure group $\left(\mathbb{R}^{+} \times \operatorname{Spin}(2)\right) \tilde{\times} \mathbb{C}$ $=\mathbb{C}^{*} \tilde{\times} \mathbb{C}$ which is first-order flat. One can check $[\mathrm{GN}]$ that the torsion conditions for this are

$$
\begin{aligned}
& T_{z \bar{z}}{ }^{z}+2 T_{\bar{z} \theta}{ }^{\theta}=0, \\
& T_{z \theta}{ }^{z}+T_{\theta \theta}{ }^{\theta}=0, \\
& T_{z \bar{\theta}}{ }^{z}+2 T_{\bar{\theta} \theta}{ }^{\theta}=0, \\
& T_{\bar{z} \theta}{ }^{z}=T_{\bar{z} \bar{\theta}}{ }^{z}=T_{\theta \bar{\theta}}{ }^{z}=T_{\overline{\theta \theta}}{ }^{z}=T_{\overline{z \theta}}{ }^{\theta}=T_{\overline{\theta \theta}}{ }^{\theta}=0, \\
& T_{\theta \theta}{ }^{z}=1,
\end{aligned}
$$

along with the complex conjugate equations. Suppose that these are satisfied. Let $P$ and $Q$ denote the canonical forms on the reduced frame bundle. Then by choosing a $\mathbb{C} \oplus \mathbb{C}$ connection with flat space torsion, it follows from Proposition 22 that it is possible to extend the connection to a flat Cartan connection. Suppose on the other hand that we have a reduction of the frame bundle of $X$ to a subbundle with structure group $\mathbb{C}^{*}$ which is not necessarily first-order flat, but satisfies the torsion conditions

$$
\begin{aligned}
& T_{\bar{z} \theta}^{z}=T_{\overline{z \theta}}^{z}=T_{\theta \bar{\theta}}^{z}=T_{\overline{\theta \theta}}^{z}=0, \\
& T_{\theta \theta}{ }^{z}=1,
\end{aligned}
$$


along with the complex conjugate equations. Let us choose a $\mathbb{C}^{*}$ connection $M$ on the reduced frame bundle so that the torsion components $T_{z \bar{z}}{ }^{z}, T_{z \theta}{ }^{z}$ and $T_{z \bar{\theta}}{ }^{z}$ vanish (this can always be done). Then by Proposition 22, it is still possible to extend $P \oplus Q \oplus M$ to a flat Cartan connection.

\section{Three Dimensions}

The superconformal algebra for three dimensional Minkowski space is osp $\mathbf{R}_{\mathbf{R}}(1 \mid 2)$. The model space $X$ is a supermanifold arising from a homogeneous $\mathbb{R}^{2}$ vector bundle over

$$
\begin{aligned}
M= & \left\{\text { real 2-planes } P \text { in } \mathbb{R}^{4}: \text { the form } d x^{0} \wedge d x^{2}+d x^{1} \wedge d x^{3}\right. \\
& \text { vanishes when pulled back to } P\} .
\end{aligned}
$$

More specifically, $X$ can be given by

$$
\begin{aligned}
X= & \left\{\mathbb{R}^{0 \mid 2} \text { planes } P \text { in } \mathbb{R}^{1 / 4}:\right. \text { the quadratic form } \\
& x^{2}+\eta_{0} \eta_{2}-\eta_{2} \eta_{0}+\eta_{1} \eta_{3}-\eta_{3} \eta_{1} \\
& \text { on } \left.\mathbb{R}^{1 / 4} \text { vanishes on } P\right\} .
\end{aligned}
$$

In order to construct a Cartan connection, let us take $h=s o(2,1) \oplus \mathbb{R}$ and $k=0$. A Cartan connection can be written in the form

$$
\omega=\left(\begin{array}{ccc}
0 & Q^{T} & -S^{T} \\
S & M & K \\
Q & P & -M^{T}
\end{array}\right),
$$

where $P$ and $K$ are real symmetric even $2 \times 2$ matrices, $M$ is an real even $2 \times 2$ matrix and $Q$ and $S$ are real odd $2 \times 1$ matrices.

Proposition 23. Given $P, Q$ and $M$ satisfying $1^{\prime}, 2^{\prime}, 3^{\prime}$ and $\Omega^{P}=0$, there are unique $S$ and $K$ such that $\Omega^{Q}=\Omega^{M}=0$.

Proof. First let us take any Cartan connection $\omega$ with the given $P, Q$ and $M$. The curvature $\Omega=d \omega+\omega \wedge \omega$ of $\omega$ has components

$$
\begin{aligned}
& \Omega^{P}=d P+Q \wedge Q^{T}+P \wedge M-M^{T} \wedge P=0, \\
& \Omega^{Q}=d Q+P \wedge S-M^{T} \wedge Q, \\
& \Omega^{M}=d M+S \wedge Q^{T}+M \wedge M+K \wedge P, \\
& \Omega^{S}=d S+M \wedge S+K \wedge Q \\
& \Omega^{K}=d K-S \wedge S^{T}+M \wedge K-K \wedge M^{T} .
\end{aligned}
$$

The Bianchi identity gives

$$
\begin{aligned}
& 0=Q \wedge\left(\Omega^{Q}\right)^{T}+P \wedge \Omega^{M}-\Omega^{Q} \wedge Q^{T}+\left(\Omega^{M}\right)^{T} \wedge P \\
& d \Omega^{Q}=P \wedge \Omega^{S}-M^{T} \wedge \Omega^{Q}+\left(\Omega^{M}\right)^{T} \wedge Q
\end{aligned}
$$


Let us write $P$ as $\tau^{\alpha \beta}, Q$ as $\tau^{\alpha}$ and $M$ as $\omega_{\alpha}^{\beta}$. Then (268), (269), (270), (273), and (274) become

$$
\begin{aligned}
& 0=d \tau^{\alpha \beta}+\tau^{\alpha} \wedge \tau^{\beta}+\tau^{\alpha \gamma} \wedge \omega_{\gamma}{ }^{\beta}-\omega_{\gamma}{ }^{\alpha} \wedge \tau^{\gamma \beta}, \\
& \left(\Omega^{Q}\right)^{\alpha}=d \tau^{\alpha}-\omega_{\beta}{ }^{\alpha} \wedge \tau^{\beta}+\tau^{\alpha \beta} \wedge S_{\beta}, \\
& \left(\Omega^{M}\right)_{\alpha}{ }^{\beta}=d \omega_{\alpha}{ }^{\beta}+S_{\alpha} \wedge \tau^{\beta}+\omega_{\alpha}{ }^{\gamma} \wedge \omega_{\gamma}{ }^{\beta}+K_{\alpha \gamma} \wedge \tau^{\gamma \beta}, \\
& 0=\tau^{\alpha} \wedge\left(\Omega^{Q}\right)^{\beta}+\tau^{\alpha \gamma} \wedge\left(\Omega^{M}\right)_{\gamma}{ }^{\beta}-\left(\Omega^{Q}\right)^{\alpha} \wedge \tau^{\beta}+\left(\Omega^{M}\right)_{\gamma}{ }^{\alpha} \wedge \tau^{\gamma \beta}, \\
& d\left(\Omega^{Q}\right)^{\alpha}=\tau^{\alpha \beta} \wedge\left(\Omega^{S}\right)_{\beta}-\omega_{\beta}{ }^{\alpha} \wedge\left(\Omega^{Q}\right)^{\beta}+\left(\Omega^{M}\right)_{\beta}{ }^{\alpha} \wedge \tau^{\beta} .
\end{aligned}
$$

Let us write $\left(\Omega^{Q}\right)^{\alpha}$ as

$$
\begin{aligned}
\left(\Omega^{Q}\right)^{\alpha}= & \frac{1}{2} \tau^{\beta} \wedge \tau^{\gamma}\left(\Omega^{Q}\right)_{\gamma, \beta}{ }^{\alpha}+\tau^{\gamma \delta} \wedge \tau^{\varepsilon}\left(\Omega^{Q}\right)_{\varepsilon, \delta \gamma}{ }^{\alpha} \\
& +\frac{1}{2} \tau^{\gamma \delta} \wedge \tau^{\varepsilon \phi}\left(\Omega^{Q}\right)_{\phi \varepsilon, \delta \gamma}{ }^{\alpha} .
\end{aligned}
$$

From (278) and (279) one can show that $\Omega^{Q}$ has the form

$$
\begin{aligned}
& \left(\Omega^{Q}\right)_{\delta, \gamma}{ }^{\beta}=0, \\
& \left(\Omega^{Q}\right)_{\varepsilon, \delta \gamma}{ }^{\alpha}=R\left(\varepsilon_{\varepsilon \delta} \delta_{\gamma}{ }^{\alpha}+\varepsilon_{\varepsilon \gamma} \delta_{\delta}^{\alpha}\right)+V_{\varepsilon \delta} \delta_{\gamma}{ }^{\alpha}+V_{\varepsilon \gamma} \delta_{\delta}{ }^{\alpha}
\end{aligned}
$$

for symmetric tensors $R$ and $V$. One can redefine $S$ so as to make $\left(\Omega^{Q}\right)_{\varepsilon, \delta \gamma}{ }^{\alpha}$ vanish. The general form for $\left(\Omega^{Q}\right)_{\phi \varepsilon, \delta \gamma}{ }^{\alpha}$ is

$$
\begin{aligned}
\left(\Omega^{Q}\right)_{\phi \varepsilon, \delta \gamma}{ }^{\alpha}= & \varepsilon_{\phi \delta} \delta_{\varepsilon}{ }^{\alpha} D_{\gamma}+\varepsilon_{\varepsilon \delta} \delta_{\phi}{ }^{\alpha} D_{\gamma}+\varepsilon_{\phi \gamma} \delta_{\varepsilon}{ }^{\alpha} D_{\delta}+\varepsilon_{\varepsilon \gamma} \delta_{\phi}{ }^{\alpha} D_{\delta} \\
& -\varepsilon_{\delta \phi} \delta_{\gamma}{ }^{\alpha} D_{\varepsilon}-\varepsilon_{\gamma \phi} \delta_{\delta}{ }^{\alpha} D_{\varepsilon}-\varepsilon_{\delta \varepsilon} \delta_{\gamma}{ }^{\alpha} D_{\phi}-\varepsilon_{\gamma \varepsilon} \delta_{\delta}{ }^{\alpha} D_{\phi} \\
& +G_{\phi \delta}{ }^{\alpha} \varepsilon_{\varepsilon \gamma}+G_{\varepsilon \delta}{ }^{\alpha} \varepsilon_{\phi \gamma}+G_{\phi \gamma}{ }^{\alpha} \varepsilon_{\varepsilon \delta}+G_{\varepsilon \gamma}{ }^{\alpha} \varepsilon_{\phi \delta}
\end{aligned}
$$

for some symmetric tensors $D$ and $G$. As in the proof of Proposition 14, by redefining $S$ we can make $\Omega^{Q}$ vanish.

The Bianchi identity (273) now becomes

$$
0=P \wedge \Omega^{M}+\left(\Omega^{M}\right)^{T} \wedge P .
$$

In components,

$$
0=\tau^{\alpha \gamma} \wedge\left(\Omega^{M}\right)_{\gamma}{ }^{\beta}+\left(\Omega^{M}\right)_{\gamma}{ }^{\alpha} \wedge \tau^{\gamma \beta}
$$

This gives

$$
\tau^{\alpha \gamma} \wedge \tau^{\varepsilon} \wedge \tau^{\delta}\left(\Omega^{M}\right)_{\delta, \varepsilon, \gamma}{ }^{\beta}+\tau^{\beta \gamma} \wedge \tau^{\varepsilon} \wedge \tau^{\delta}\left(\Omega^{M}\right)_{\delta, \varepsilon, \gamma}{ }^{\alpha}=0,
$$

which implies that $\left(\Omega^{M}\right)_{\delta, \varepsilon, \gamma}{ }^{\beta}$ vanishes. Also,

$$
\tau^{\alpha \gamma} \wedge \tau^{\phi \varepsilon} \wedge \tau^{\delta}\left(\Omega^{M}\right)_{\delta, \varepsilon \phi, \gamma}{ }^{\beta}+\tau^{\beta \gamma} \wedge \tau^{\phi \varepsilon} \wedge \tau^{\delta}\left(\Omega^{M}\right)_{\delta, \varepsilon \phi, \gamma}{ }^{\alpha}=0 .
$$

It can be verfied that the most general solution to (287) is of the form

$$
\left(\Omega^{M}\right)_{\delta, \varepsilon \phi, \gamma}{ }^{\beta}=N_{\delta \gamma \phi} \delta_{\varepsilon}{ }^{\beta}+N_{\delta \gamma \varepsilon} \delta_{\phi}{ }^{\beta}
$$

for some tensor $N_{\delta \gamma \phi}$ which is symmetric in the last two indices. From Eq. (277), $K_{\delta, \alpha \gamma}$ can be redefined in order to make $\left(\Omega^{M}\right)_{\delta, \varepsilon \phi, \gamma}{ }^{\beta}$ vanish. Finally, because the Weyl tensor vanishes in three dimensions, $K_{\delta \varepsilon, \alpha \gamma}$ can be redefined in order to make $\left(\Omega^{M}\right)_{\delta \varepsilon, \phi \ell, \gamma}{ }^{\beta}$ vanish. 
Note that the remaining curvature components, $\Omega^{S}$ and $\Omega^{K}$ are uniquely determined and generally nonvanishing. For a superRiemannian geometry, they give the supersymmetric analog of the three-dimensional conformal tensor [Ei].

\section{Four Dimensions}

The superconformal algebra for four dimensional Minkowski space is $s u(1 \mid 2,2)$. The model space $X$ is a supermanifold arising from a homogeneous $\mathbb{R}^{4}$ vector bundle over

$$
\begin{aligned}
M= & \left\{\text { complex 2-planes } P \text { in } \mathbb{C}^{4}:\right. \text { the form } \\
& d \bar{x}_{0} \wedge d x_{2}-d \bar{x}_{2} \wedge d x_{0}+d \bar{x}_{1} \wedge d x_{3}-d \bar{x}_{3} \wedge d x_{1} \\
& \text { vanishes when pulled back to } P\} .
\end{aligned}
$$

More specifically, $X$ can be given by

$$
\begin{aligned}
X= & \left\{\mathbb{C}^{0 / 2} \text { planes } P \text { in } \mathbb{C}^{1 / 4}:\right. \text { the quadratic form } \\
& \bar{x} x+\bar{\eta}_{0} \eta_{2}-\bar{\eta}_{2} \eta_{0}+\bar{\eta}_{1} \eta_{3}-\bar{\eta}_{3} \eta_{1} \\
& \text { on } \left.\mathbb{C}^{1 / 4} \text { vanishes on } P\right\} .
\end{aligned}
$$

In order to construct a Cartan connection, let us take $h=s o(3,1) \oplus \mathbb{R}$ and $k=u(1)$. A Cartan connection can be written in the form

$$
\omega=\left(\begin{array}{llr}
A & Q^{\dagger} & -S^{T} \\
S^{*} & M^{*} & K^{*} \\
Q & P & -M^{T}
\end{array}\right),
$$

where $A$ is imaginary, $P$ and $K$ are Hermitian even $2 \times 2$ matrices, $M$ is a complex even $2 \times 2$ matrix and $Q$ and $S$ are complex odd $2 \times 1$ matrices.

Proposition 24. Given $P, Q$ and $M$ satisfying $1^{\prime}, 2^{\prime}, 3^{\prime}$ and $\Omega^{P}=0$, there are unique $A, S$ and $K$ such that $\Omega^{Q}$ has the form

$$
\left(\Omega^{Q}\right)^{\alpha}=\tau^{\gamma \dot{\delta}} \wedge \tau^{\varepsilon \dot{\phi}} O_{\dot{\phi} \dot{\delta} \varepsilon \gamma^{\alpha}},
$$

where $O_{\phi \delta \varepsilon \gamma}{ }^{\alpha}$ is skew in $\phi$ and $\dot{\delta}$, symmetric in $\varepsilon$ and $\gamma$ and $O_{\phi \delta \varepsilon \alpha}{ }^{\alpha}=0$, and $\Omega^{M}$ has the form

$$
\left(\Omega^{M}\right)_{\alpha}{ }^{\beta}=\tau^{\gamma \dot{\delta}} \wedge \tau^{\dot{\varepsilon}} X_{\dot{\varepsilon} \dot{\delta} \gamma \alpha}{ }^{\beta}+\tau^{\gamma \dot{\delta}} \wedge \tau^{\varepsilon \dot{\phi}} Z_{\phi \dot{\delta} \varepsilon \gamma \alpha}{ }^{\beta},
$$

where $X_{\dot{\varepsilon} \delta \beta \alpha}{ }^{\beta}=0, Z_{\dot{\phi} \delta \varepsilon \gamma \alpha}{ }^{\beta}=-Z_{\delta \phi \gamma \varepsilon \alpha}{ }^{\beta}$ and $Z_{\phi \dot{\phi} \varepsilon \beta \alpha}{ }^{\beta}=0$.

Proof. First let us take any Cartan connection $\omega$ with the given $P, Q$ and $M$. The curvature $\Omega=d \omega+\omega \wedge \omega$ of $\omega$ has components

$$
\begin{aligned}
& \Omega^{P}=d P+Q \wedge Q^{\dagger}+P \wedge M^{*}-M^{T} \wedge P=0 \\
& \Omega^{Q}=d Q+Q \wedge A+P \wedge S^{*}-M^{T} \wedge Q \\
& \Omega^{M}=d M+S \wedge Q^{T}+M \wedge M+K \wedge P^{T} \\
& \Omega^{A}=d A+Q^{\dagger} \wedge S^{*}-S^{T} \wedge Q \\
& \Omega^{S}=d S+S \wedge A^{*}+M \wedge S+K \wedge Q^{*} \\
& \Omega^{K}=d K-S \wedge S^{\dagger}+M \wedge K-K \wedge M^{\dagger}
\end{aligned}
$$


The Bianchi identity gives

$$
\begin{aligned}
& 0=Q \wedge\left(\Omega^{Q}\right)^{\dagger}+P \wedge\left(\Omega^{M}\right)^{*}-\Omega^{Q} \wedge Q^{\dagger}+\left(\Omega^{M}\right)^{T} \wedge P, \\
& d \Omega^{Q}=Q \wedge \Omega^{A}+P \wedge\left(\Omega^{S}\right)^{*}-M^{T} \wedge \Omega^{Q}-\Omega^{Q} \wedge A+\left(\Omega^{M}\right)^{T} \wedge Q .
\end{aligned}
$$

Let us write $P$ as $\tau^{\alpha \dot{\beta}}, Q$ as $\tau^{\alpha}$ and $M$ as $\omega_{\alpha}{ }^{\beta}$. Then (293), (294), (295), (299), and (300) become

$$
\begin{aligned}
& 0=d \tau^{\alpha \dot{\beta}}+\tau^{\alpha} \wedge \tau^{\dot{\beta}}+\tau^{\alpha \gamma} \wedge \omega_{\dot{\gamma}}^{\dot{\beta}}-\omega_{\gamma}{ }^{\alpha} \wedge \tau^{\gamma \dot{\beta}} \\
& \left(\Omega^{Q}\right)^{\alpha}=d \tau^{\alpha}-\omega_{\beta}{ }^{\alpha} \wedge \tau^{\beta}+\tau^{\alpha} \wedge A+\tau^{\alpha \dot{\beta}} \wedge S_{\dot{\beta}}, \\
& \left(\Omega^{M}\right)_{\alpha}{ }^{\beta}=d \omega_{\alpha}{ }^{\beta}+S_{\alpha} \wedge \tau^{\beta}+\omega_{\alpha}{ }^{\gamma} \wedge \omega_{\gamma}{ }^{\beta}+K_{\alpha \dot{\gamma}} \wedge \tau^{\beta \gamma}, \\
& 0=\tau^{\alpha} \wedge\left(\Omega^{Q}\right)^{\dot{\beta}}+\tau^{\alpha \dot{\gamma}} \wedge\left(\Omega^{M}\right)_{\dot{\gamma}}^{\dot{\beta}}-\left(\Omega^{Q}\right)^{\alpha} \wedge \tau^{\dot{\beta}}+\left(\Omega^{M}\right)_{\gamma}{ }^{\alpha} \wedge \tau^{\gamma \dot{\beta}}, \\
& d\left(\Omega^{Q}\right)^{\alpha}=\tau^{\alpha} \wedge \Omega^{A}+\tau^{\alpha \dot{\beta}} \wedge\left(\Omega^{S}\right)_{\dot{\beta}}-\omega_{\beta}{ }^{\alpha} \wedge\left(\Omega^{Q}\right)^{\beta}-\left(\Omega^{Q}\right)^{\alpha} \wedge A \\
& \quad+\left(\Omega^{M}\right)_{\beta}{ }^{\alpha} \wedge \tau^{\beta} .
\end{aligned}
$$

Let us write $\left(\Omega^{Q}\right)^{\alpha}$ as

$$
\begin{aligned}
\left(\Omega^{Q}\right)^{\alpha}= & \frac{1}{2} \tau^{\beta} \wedge \tau^{\gamma}\left(\Omega^{Q}\right)_{\gamma, \beta}{ }^{\alpha}+\tau^{\beta} \wedge \tau^{\gamma}\left(\Omega^{Q}\right)_{\dot{\gamma}, \beta}{ }^{\alpha} \\
& +\frac{1}{2} \tau^{\dot{\beta}} \wedge \tau^{\gamma}\left(\Omega^{Q}\right)_{\dot{\gamma},{ }^{\alpha}}+\tau^{\gamma \dot{\delta}} \wedge \tau^{\varepsilon}\left(\Omega^{Q}\right)_{\varepsilon, \dot{\delta} \gamma}{ }^{\alpha}+\tau^{\gamma \dot{\delta}} \wedge \tau^{\dot{\varepsilon}}\left(\Omega^{Q}\right)_{\dot{\varepsilon}, \dot{\delta} \gamma}{ }^{\alpha} \\
& +\frac{1}{2} \tau^{\gamma \dot{\delta}} \wedge \tau^{\varepsilon \dot{\phi}}\left(\Omega^{Q}\right)_{\phi \varepsilon, \dot{\gamma} \gamma}{ }^{\alpha} .
\end{aligned}
$$

From (304) one can show

$$
\left(\Omega^{Q}\right)_{\dot{\gamma}, \beta}^{\alpha}=0, \quad\left(\Omega^{Q}\right)_{\dot{\gamma}, \beta}^{\alpha}=V_{\dot{\gamma}} \delta_{\beta}^{\alpha} \quad \text { and } \quad\left(\Omega^{Q}\right)_{\gamma, \beta}^{\alpha}=V_{\gamma} \delta_{\beta}^{\alpha}+V_{\beta} \delta_{\gamma}^{\alpha},
$$

where $\left(V_{\dot{\gamma}}\right)^{*}=-V_{\gamma}$. Then by redefining $A$ we can assume

$$
\left(\Omega^{Q}\right)_{\dot{\gamma}, \beta^{\alpha}}=\left(\Omega^{Q}\right)_{\dot{\gamma}, \beta}^{\alpha}=\left(\Omega^{Q}\right)_{\gamma, \beta}{ }^{\alpha}=0 \text {. }
$$

The general forms for $\left(\Omega^{Q}\right)_{\varepsilon, \dot{\delta}_{\gamma}}{ }^{\alpha}$ and $\left(\Omega^{Q}\right)_{\dot{\varepsilon}, \delta_{\gamma}}{ }^{\alpha}$ are

and

$$
\left(\Omega^{Q}\right)_{\varepsilon, \dot{\delta} \gamma}{ }^{\alpha}=H_{\dot{\delta}_{\varepsilon \gamma}}{ }^{\alpha}+I_{\varepsilon \dot{\delta}} \delta_{\gamma}^{\alpha}+J_{\gamma \dot{\delta}} \delta_{\varepsilon}^{\alpha}
$$

$$
\left(\Omega^{Q}\right)_{\dot{\varepsilon}, \delta \gamma}{ }^{\alpha}=T_{\dot{\varepsilon} \delta} \delta_{\gamma}^{\alpha}+U_{\dot{\varepsilon} \dot{\delta} \gamma}^{\alpha},
$$

where $H_{\delta \varepsilon \gamma}{ }^{\alpha}$ is symmetric in $\varepsilon$ and $\gamma$ and $H_{\delta \varepsilon \alpha}{ }^{\alpha}=0$, and $U_{\dot{\varepsilon} \delta \alpha}{ }^{\alpha}=0$. We can redefine $S$ in order to make $I$ and $T$ vanish. We can redefine $A$ to make the skew-Hermitian part of $J$ vanish.

The general form for $\left(\Omega^{Q}\right)_{\phi \varepsilon, \delta \gamma}{ }^{\alpha}$ is

$$
\left(\Omega^{Q}\right)_{\phi \varepsilon, \delta \gamma}{ }^{\alpha}=B_{\phi \delta \varepsilon} \delta_{\gamma}^{\alpha}+B_{\phi \delta \gamma} \delta_{\varepsilon}^{\alpha}+N_{\phi \delta \varepsilon} \delta_{\gamma}^{\alpha}-N_{\phi \delta \gamma} \delta_{\varepsilon}^{\alpha}+O_{\phi \delta \varepsilon \gamma}{ }^{\alpha},
$$

where $B_{\phi \delta_{\varepsilon}}$ is skew in $\dot{\phi}$ and $\delta, N_{\phi \delta \varepsilon}$ is symmetric in $\dot{\phi}$ and $\dot{\delta}, O_{\phi \delta \varepsilon \gamma}{ }^{\alpha}$ is skew in $\dot{\phi}$ and $\dot{\delta}$, symmetric in $\varepsilon$ and $\gamma$ and $O_{\phi \delta \varepsilon \alpha}{ }^{\alpha}=0$. By redefining $S$, we can make $B$ and $N$ vanish. $A$ and $S$ are now uniquely determined.

The general forms for $\left(\Omega^{M}\right)_{\varepsilon, \delta \gamma, \alpha}{ }^{\beta}$ and $\left(\Omega^{M}\right)_{\dot{\varepsilon}, \delta \gamma, \alpha}{ }^{\beta}$ are

$$
\begin{aligned}
& \left(\Omega^{M}\right)_{\varepsilon, \dot{\delta} \gamma, \alpha}{ }^{\beta}=R_{\varepsilon \delta \alpha} \delta_{\gamma}{ }^{\beta}+V_{\varepsilon \dot{\delta} \gamma \alpha}{ }^{\beta}, \\
& \left(\Omega^{M}\right)_{\dot{\varepsilon}, \delta \gamma, \alpha}{ }^{\beta}=W_{\dot{\varepsilon} \delta \alpha} \delta_{\gamma}{ }^{\beta}+X_{\dot{\varepsilon} \delta \gamma \alpha}{ }^{\beta},
\end{aligned}
$$


where $V_{\varepsilon \dot{\delta} \beta \alpha}{ }^{\beta}=X_{\dot{\varepsilon} \delta \beta \alpha}{ }^{\beta}=0$. By redefining $K$, we can make $R$ and $W$ vanish. The general form for $\left(\Omega^{M}\right)_{\phi \varepsilon, \delta \gamma, \alpha}{ }^{\beta}$ is

$$
\left(\Omega^{M}\right)_{\dot{\phi} \varepsilon, \dot{\delta} \gamma, \alpha}{ }^{\beta}=Y_{\dot{\phi} \dot{\delta} \varepsilon \alpha} \delta_{\gamma}{ }^{\beta}-Y_{\delta \dot{\phi} \gamma \alpha} \delta_{\varepsilon}{ }^{\beta}+Z_{\dot{\phi} \dot{\delta} \varepsilon \alpha{ }^{\beta}}{ }^{\beta},
$$

where $Z_{\phi \delta \varepsilon \gamma \alpha}{ }^{\beta}=-Z_{\delta \phi \gamma \varepsilon \alpha}{ }^{\beta}$ and $Z_{\phi \delta \varepsilon \beta \alpha}{ }^{\beta}=0$. By redefining $K$, we can make $Y$ vanish. $K$ is now uniquely determined.

From the Bianchi identities (304) and (305) one finds that $H, J, U$ and $V$ vanish. Thus $\Omega^{Q}$ and $\Omega^{M}$ are as stated in the proposition.

Let us note that using the Bianchi identities, $X$ and $Z$ can be given explicitly in terms of $O$ (see [Ho2]). $O$ is the super analog of the Weyl tensor.

\section{E. Six Dimensions}

The superconformal algebra for six dimensional Minkowski space is hosp (1|2). The model space $X$ is a supermanifold arising from a homogeneous $\mathbb{H}^{2}$ vector bundle over

$$
\begin{aligned}
M= & \left\{\text { quaternionic 2-planes } P \text { in } \mathrm{H}^{4}:\right. \text { the form } \\
& \overline{d x^{0}} \wedge d x^{2}-\overline{d x^{2}} \wedge d x^{0}+\overline{d x^{1}} \wedge d x^{3}-\overline{d x^{3}} \wedge d x^{1} \\
& \text { vanishes when pulled back to } P\} .
\end{aligned}
$$

More specifically, $X$ can be given by

$X=\left\{\mathrm{H}^{0 \mid 2}\right.$ planes $P$ in $\mathbb{H}^{1 / 4}$ : the quadratic form

$$
\left.\bar{x} x+\overline{\eta_{0}} \eta_{2}-\overline{\eta_{2}} \eta_{0}+\overline{\eta_{1}} \eta_{3}-\overline{\eta_{3}} \eta_{1} \text { on } \mathbb{H}^{1 / 4} \text { vanishes on } P\right\} \text {. }
$$

For calculations, it is convenient to use an equivalent form of hosp (1/2). Let $J$ be a real skew-symmetric operator on $\mathbb{C}^{4}, J^{2}=-1$, and let $\varepsilon$ be a real skew-symmetric operator on $\mathbb{C}^{2}, \varepsilon^{2}=-1$. Let $B$ and $C$ be the operators

$$
B=\left(\begin{array}{rrr}
\varepsilon & 0 & 0 \\
0 & J & 0 \\
0 & 0 & -J
\end{array}\right) \text { and } C=\left(\begin{array}{ccc}
\varepsilon & 0 & 0 \\
0 & 0 & I \\
0 & I & 0
\end{array}\right)
$$

on $\mathbb{C}^{2} \oplus \mathbb{C}^{4} \oplus \mathbb{C}^{4}$. Then

$$
\operatorname{hosp}(1 \mid 2) \approx s o^{*}(2 \mid 8)=\left\{M \in g l\left(\mathbb{C}^{2 / 8}\right): M B=B \bar{M} \text { and } M^{T} C+C M=0\right\} .
$$

In order to construct a Cartan connection, let us take $h=s o(5,1) \oplus \mathbb{R}$ and $k=s u(2)$. A Cartan connection can be written in the form

$$
\omega=\left(\begin{array}{ccc}
A & \varepsilon Q^{T} & \varepsilon S^{T} \\
S & M & K \\
Q & P & -M^{T}
\end{array}\right),
$$

where $A$ is a skew-Hermitian $2 \times 2$ matrix, $P$ and $K$ are complex skew-symmetric even $4 \times 4$ matrices such that $P J=-J \bar{P}$ and $K J=-J \bar{K}, M$ is an complex even $4 \times 4$ matrix such that $M J=J \bar{M}$, and $Q$ and $S$ are complex odd $4 \times 2$ matrices such that $Q \varepsilon=-J \bar{Q}$ and $S \varepsilon=-J \bar{S}$. 
Proposition 25. Given $P, Q$, and $M$ satisfying $1^{\prime}, 2^{\prime}, 3^{\prime}$ and $\Omega^{P}=0$, there are unique $A$, $S$ and $K$ such that $\Omega^{Q}$ has the form

$$
\left(\Omega^{Q}\right)_{i}^{\alpha}=\tau^{\gamma \delta} \wedge \tau_{i}^{\varepsilon} N_{\varepsilon \delta \gamma}^{\alpha}+\frac{1}{2} \tau^{\gamma \delta} \wedge \tau^{\varepsilon \phi} R_{\phi \varepsilon \delta \gamma i}^{\alpha},
$$

where $N_{\varepsilon \delta \gamma}{ }^{\alpha}$ is skew in $\varepsilon \delta \gamma$,

$$
\begin{aligned}
& N_{\varepsilon \delta \alpha}^{\alpha}=0, \\
& R_{\phi \varepsilon \delta \gamma i}^{\alpha}=-R_{\varepsilon \phi \delta \gamma i}^{\alpha}=-R_{\phi \varepsilon \gamma \delta i}^{\alpha}=-R_{\delta \gamma \phi \varepsilon i}^{\alpha} \text { and } R_{\phi \varepsilon \delta \alpha i}^{\alpha}=0,
\end{aligned}
$$

and $\Omega^{M}$ has the form

where

$$
\begin{aligned}
\left(\Omega^{M}\right)_{\alpha}{ }^{\beta}= & \tau^{\beta \delta} \wedge \tau_{j}^{\varepsilon} T_{\varepsilon \delta \alpha}^{j}+\tau^{\gamma \delta} \wedge \tau_{j}^{\varepsilon} Z_{\varepsilon \delta \gamma \alpha}^{j}{ }^{\beta}+\tau^{\beta \delta} \wedge \tau^{\varepsilon \phi} C_{\phi \varepsilon \delta \alpha} \\
& +\tau^{\gamma \delta} \wedge \tau^{\varepsilon \phi} D_{\phi \varepsilon \delta \gamma \alpha}{ }^{\beta}
\end{aligned}
$$

$$
\begin{aligned}
& T_{\varepsilon \delta \alpha}^{j}=T_{\varepsilon \alpha \delta}^{j}, \\
& Z_{\varepsilon \delta \gamma \alpha}^{j}{ }^{\beta}=-Z_{\varepsilon \gamma \delta \alpha}^{j}{ }^{\beta}, Z_{\varepsilon \delta \beta \alpha}^{j}{ }^{\beta}=0, \\
& C_{\phi \varepsilon \gamma \alpha}=-C_{\varepsilon \phi \gamma \alpha}=C_{\phi \varepsilon \alpha \gamma}, \\
& D_{\phi \varepsilon \delta \gamma \alpha}{ }^{\beta}=-D_{\varepsilon \phi \delta \gamma \alpha}{ }^{\beta}=-D_{\phi \varepsilon \gamma \delta \alpha}{ }^{\beta}=-D_{\delta \gamma \phi \varepsilon \alpha}{ }^{\beta} \text { and } D_{\phi \varepsilon \delta \beta \alpha}{ }^{\beta}=0 .
\end{aligned}
$$

Proof. First let us take any Cartan connection $\omega$ with the given $P, Q$ and $M$. The curvature $\Omega=d \omega+\omega \wedge \omega$ of $\omega$ has components

$$
\begin{aligned}
& \Omega^{P}=d P+Q \wedge \varepsilon Q^{T}+P \wedge M-M^{T} \wedge P=0 \\
& \Omega^{Q}=d Q+Q \wedge A+P \wedge S-M^{T} \wedge Q \\
& \Omega^{M}=d M+S \wedge \varepsilon Q^{T}+M \wedge M+K \wedge P \\
& \Omega^{A}=d A+A \wedge A+\varepsilon Q^{T} \wedge S+\varepsilon S^{T} \wedge Q \\
& \Omega^{S}=d S+S \wedge A+M \wedge S+K \wedge Q \\
& \Omega^{K}=d K+S \wedge \varepsilon S^{T}+M \wedge K-K \wedge M^{T}
\end{aligned}
$$

The Bianchi identity gives

$$
\begin{aligned}
& 0=Q \wedge \varepsilon\left(\Omega^{Q}\right)^{T}+P \wedge \Omega^{M}-\Omega^{Q} \wedge \varepsilon Q^{T}+\left(\Omega^{M}\right)^{T} \wedge P, \\
& d \Omega^{Q}=Q \wedge \Omega^{A}-\Omega^{Q} \wedge A+P \wedge \Omega^{S}-M^{T} \wedge \Omega^{Q}+\left(\Omega^{M}\right)^{T} \wedge Q .
\end{aligned}
$$

Let us write $P$ as $\tau^{\alpha \beta}, Q$ as $\tau_{i}^{\alpha}$ and $M$ as $\omega_{\alpha}{ }^{\beta}$. Then (326), (327), (328), (332), and (333) become

$$
\begin{aligned}
& 0=d \tau^{\alpha \beta}+\varepsilon^{i j} \tau_{i}^{\alpha} \wedge \tau_{j}^{\beta}+\tau^{\alpha \gamma} \wedge \omega_{\gamma}{ }^{\beta}-\omega_{\gamma}{ }^{\alpha} \wedge \tau^{\gamma \beta}, \\
& \left(\Omega^{Q}\right)_{i}^{\alpha}=d \tau_{i}^{\alpha}+\tau_{j}^{\alpha} \wedge A_{i}^{j}-\omega_{\beta}{ }^{\alpha} \wedge \tau_{i}^{\beta}+\tau^{\alpha \beta} \wedge S_{\beta i}, \\
& \left(\Omega^{M}\right)_{\alpha}{ }^{\beta}=d \omega_{\alpha}{ }^{\beta}+\varepsilon^{i j} S_{\alpha i} \wedge \tau_{j}^{\beta}+\omega_{\alpha}{ }^{\gamma} \wedge \omega_{\gamma}{ }^{\beta}+K_{\alpha \gamma} \wedge \tau^{\gamma \beta}, \\
& 0=\varepsilon^{i j} \tau_{i}^{\alpha} \wedge\left(\Omega^{Q}\right)_{j}^{\beta}+\tau^{\alpha \gamma} \wedge\left(\Omega^{M}\right)_{\gamma}{ }^{\beta}-\varepsilon^{i j}\left(\Omega^{Q}\right)_{i}^{\alpha} \wedge \tau_{j}^{\beta} \\
& \quad+\left(\Omega^{M}\right)_{\gamma}{ }^{\alpha} \wedge \tau^{\gamma \beta} \\
& d\left(\Omega^{Q}\right)_{i}^{\alpha}=\tau_{j}^{\alpha} \wedge\left(\Omega^{A}\right)_{i}^{j}-\left(\Omega^{Q}\right)_{j}^{\alpha} \wedge A^{j}{ }_{i}+\tau^{\alpha \beta} \wedge\left(\Omega^{S}\right)_{\beta i} \\
& \quad-\omega_{\beta}{ }^{\alpha} \wedge\left(\Omega^{Q}\right)_{i}{ }^{\beta}+\left(\Omega^{M}\right)_{\beta}{ }^{\alpha} \wedge \tau_{i}^{\beta} .
\end{aligned}
$$


Let us write $\left(\Omega^{Q}\right)_{i}^{\alpha}$ as

$$
\begin{aligned}
\left(\Omega^{Q}\right)_{i}^{\alpha}= & \frac{1}{2} \tau_{j}^{\beta} \wedge \tau_{k}^{\gamma}\left(\Omega^{Q}\right)_{\gamma, \beta i}^{k j \alpha}+\tau^{\gamma \delta} \wedge \tau_{j}^{\varepsilon}\left(\Omega^{Q}\right)_{\varepsilon, \delta \gamma i}^{j} \alpha \\
& +\frac{1}{2} \tau^{\gamma \delta} \wedge \tau^{\varepsilon \phi}\left(\Omega^{Q}\right)_{\phi \varepsilon, \delta \gamma i}^{\alpha} .
\end{aligned}
$$

From (337) one can show that $\left(\Omega^{Q}\right)_{\gamma, \beta i}^{k} j$ has the form

$$
\left(\Omega^{Q}\right)_{\gamma, \beta i}^{k j \alpha}=V_{\gamma}^{k j}{ }_{i} \delta_{\beta}{ }^{\alpha}+V_{\beta}^{k j}{ }_{i} \delta_{\gamma}^{\alpha},
$$

where $V_{\gamma}^{k j}{ }_{i}$ is symmetric in $k$ and $j$, and $V_{\gamma}^{k j}{ }_{j}=0$. By redefining $A$, we can make $\left(\Omega^{Q}\right)_{\gamma, \beta i}^{k j \alpha}$ vanish.

The general form for $\left(\Omega^{Q}\right)_{\varepsilon, \delta \gamma i}^{j}{ }^{\alpha}$ is

where

$$
\begin{aligned}
\left(\Omega^{Q}\right)_{\varepsilon, \delta \gamma i}^{j}= & \delta_{\varepsilon}{ }^{\alpha} W_{\delta \gamma i}{ }^{j}+\delta_{\varepsilon}{ }^{\alpha} Y_{\delta \gamma} \delta^{j}{ }_{i}+\delta_{\gamma}{ }^{\alpha} X_{\varepsilon \delta i}^{j} \\
& -\delta_{\delta}{ }^{\alpha} X_{\varepsilon \gamma i}^{j}+M_{\varepsilon, \delta \gamma i}^{j}{ }^{\alpha}
\end{aligned}
$$

$$
\begin{aligned}
& W_{\delta \gamma i}^{i}=M_{\alpha, \delta \gamma i}^{j}=M_{\varepsilon, \delta \alpha i}^{j}=0, \quad Y_{\delta \gamma}=-Y_{\gamma \delta}, \\
& M_{\varepsilon, \delta \gamma i}^{j}=-M_{\varepsilon, \gamma \delta i}^{j}{ }^{\alpha} .
\end{aligned}
$$

By redefining $A$ and $S$, we may assume that $W$ and $X$ vanish. $A$ is now uniquely determined. From (337) and (338), one can show that $Y$ vanishes and that

$$
M_{\varepsilon, \delta \gamma i}^{j}=N_{\varepsilon \delta \gamma}^{\alpha}{ }^{j}{ }_{i}
$$

for some tensor $N_{\varepsilon \delta \gamma}{ }^{\alpha}$ which is completely antisymmetric in $\varepsilon \delta \gamma$ and satisfies $N_{\varepsilon \delta \alpha}^{\alpha}=0$.

The general form for $\left(\Omega^{Q}\right)_{\phi \varepsilon, \delta \gamma i}^{\alpha}$ is

where

$$
\begin{aligned}
\left(\Omega^{Q}\right)_{\phi \varepsilon, \delta \gamma i}^{\alpha}= & \delta_{\phi}^{\alpha} O_{\varepsilon \delta \gamma i}-\delta_{\varepsilon}^{\alpha} O_{\phi \delta \gamma i}-\delta_{\delta}^{\alpha} O_{\gamma \phi \varepsilon i}+\delta_{\gamma}^{\alpha} O_{\delta \phi \varepsilon i} \\
& +R_{\phi \varepsilon \delta \gamma i}^{\alpha},
\end{aligned}
$$

$$
\begin{aligned}
& O_{\varepsilon \delta \gamma i}=-O_{\varepsilon \gamma \delta i}, \quad R_{\phi \varepsilon \delta \gamma i}^{\alpha}=-R_{\varepsilon \phi \delta \gamma i}^{\alpha}=-R_{\phi \varepsilon \gamma \delta i}^{\alpha}=-R_{\delta \gamma \phi \varepsilon i}^{\alpha}, \\
& R_{\phi \varepsilon \delta \alpha i}^{\alpha}=0 .
\end{aligned}
$$

By redefining $S$, we may assume that $O$ vanishes. $S$ is now uniquely determined. One can show that (337) and (338) give no further conditions on $R$.

The general form for $\left(\Omega^{M}\right)_{\varepsilon, \delta \gamma, i \alpha}^{j}$ is

$$
\left(\Omega^{M}\right)_{\varepsilon, \delta \gamma, \alpha}^{j}{ }^{\beta}=T_{\varepsilon \delta \alpha}^{j} \delta_{\gamma}{ }^{\beta}-T_{\varepsilon \gamma \alpha}^{j} \delta_{\delta}{ }^{\beta}+U_{\varepsilon \delta \alpha}^{j} \delta_{\gamma}{ }^{\beta}-U_{\varepsilon \gamma \alpha}^{j} \delta_{\delta}{ }^{\beta}+Z_{\varepsilon \delta \gamma \alpha}^{j}{ }^{\beta},
$$

where

$$
T_{\varepsilon \delta \alpha}^{j}=T_{\varepsilon \alpha \delta}^{j}, \quad U_{\varepsilon \delta \alpha}^{j}=-U_{\varepsilon \alpha \delta}^{j}, \quad Z_{\varepsilon \delta \gamma \alpha}^{j}{ }^{\beta}=-Z_{\varepsilon \gamma \delta \alpha}^{j}{ }^{\beta}, \quad Z_{\varepsilon \delta \beta \alpha}^{j}{ }^{\beta}=0 .
$$

We can redefine $K$ in order to make $U$ vanish.

The general form for $\left(\Omega^{M}\right)_{\phi \varepsilon, \delta \gamma, \alpha}{ }^{\beta}$ is

$$
\begin{aligned}
\left(\Omega^{M}\right)_{\phi \varepsilon, \delta \gamma, \alpha}{ }^{\beta}= & B_{\phi \varepsilon \gamma \alpha} \delta_{\delta}{ }^{\beta}-B_{\phi \varepsilon \delta \alpha} \delta_{\gamma}{ }^{\beta}-B_{\delta \gamma \varepsilon \alpha} \delta_{\phi}{ }^{\beta}+B_{\delta \gamma \phi \alpha} \delta_{\varepsilon}{ }^{\beta} \\
& +C_{\phi \varepsilon \gamma \alpha} \delta_{\delta}{ }^{\beta}-C_{\phi \varepsilon \delta \alpha} \delta_{\gamma}{ }^{\beta}-C_{\delta \gamma \varepsilon \alpha} \delta_{\phi}{ }^{\beta} \\
& +C_{\delta \gamma \phi \alpha} \delta_{\varepsilon}{ }^{\beta}+D_{\phi \varepsilon \delta \gamma \alpha}{ }^{\beta}
\end{aligned}
$$


where

$$
\begin{aligned}
& B_{\phi \varepsilon \gamma \alpha}=-B_{\varepsilon \phi \gamma \alpha}=-B_{\phi \varepsilon \alpha \gamma}, \quad C_{\phi \varepsilon \gamma \alpha}=-C_{\varepsilon \phi \gamma \alpha}=C_{\phi \varepsilon \alpha \gamma}, \\
& D_{\phi \varepsilon \delta \gamma \alpha}{ }^{\beta}=-D_{\varepsilon \phi \delta \gamma \alpha}{ }^{\beta}=-D_{\phi \varepsilon \gamma \delta \alpha}{ }^{\beta}=-D_{\delta \gamma \phi \varepsilon \alpha}{ }^{\beta}, \quad D_{\phi \varepsilon \delta \beta \alpha}{ }^{\beta}=0 .
\end{aligned}
$$

We redefine $K$ in order to make $B$ vanish. $K$ is now uniquely defined.

We note that using the Bianchi identities, $T, Z, C$, and $D$ can be given in terms of $N$ and $R$, although we do not bother to do so explicitly.

\section{SuperKähler Manifolds}

Suppose that we have a supermanifold $X$ with a reduction of its frame bundle to a superRiemannian structure bundle. If we want to define a superKähler structure on $X$, a reasonable necessary condition is that the reduced manifold $M^{2 n}$ should have a Kähler structure, that is, the frame bundle of $M$ should have a reduction to a firstorder flat $U(n)$ subbundle. Thus the structure group of a superKähler structure should be a subgroup of $(\operatorname{Spin}(2 n) \times K) \tilde{\times} \mathscr{S}$ whose even part is $U(1) \times S U(n) \times K$, the double cover of $U(n) \times K$. A natural such subgroup can be constructed by using the fact that the spinors on a Kähler manifold can be identified with twisted $\Lambda^{*, 0}$ forms [Hi]. The spinor representation

$$
\varrho: U(1) \times S U(n) \rightarrow \operatorname{End}\left(\Lambda^{p, 0} \mathbb{C}\right)
$$

is the tensor product of the representation of $U(1)$ on $\mathbb{C}$ given by $e^{i \theta} \rightarrow e^{2(p-n / 2) i \theta}$ and the representation of $S U(n)$ on $\Lambda^{p, 0}$ which is derived from the representation on $\Lambda^{1,0}$.

Let $\pi$ denote the standard representation of $u(n)$ on $\mathbb{C}^{n}$. Let $\left\{e_{\alpha}\right\}_{\alpha=1}^{n}$ be the standard basis for $\mathbb{C}^{n}$, with dual basis $\left\{\tau^{\alpha}\right\}_{\alpha=1}^{n}$. Let $\{\eta \alpha\}$ be a standard basis for $\Lambda^{*, 0}$, where $a$ runs over a set of $2^{n}$ multi-indices. Given an element $Q$ of $\Lambda^{*, 0}$, let us write it as $Q=\sum_{a} Q_{a} \eta^{a}$.

Definition 5. The superunitary algebra $u$ on $\mathbb{C}^{n \mid 2^{n}}$ is the real super Lie algebra with even part $u(n) \oplus \mathbb{C}^{n}$ and odd part $\mathbb{C}^{2^{n}}$, with commutation relations

$$
\begin{aligned}
& {\left[M \oplus P, M^{\prime} \oplus P^{\prime}\right]=\left[M, M^{\prime}\right] \oplus\left(\pi(M) P^{\prime}-\pi\left(M^{\prime}\right) P\right) \oplus 0,} \\
& {[M \oplus P, Q]=0 \oplus 0 \oplus \varrho(M) Q,} \\
& {\left[Q, Q^{\prime}\right]=0 \oplus \sum_{\alpha=1}^{n} \sum_{a}\left(\overline{Q_{a}} Q_{\tau^{\alpha} \wedge a}^{\prime}+\overline{Q^{\prime}{ }_{a}} Q_{\tau^{\alpha} \wedge a}\right) e_{\alpha} \oplus 0 .}
\end{aligned}
$$

If we denote the corresponding superunitary Lie group by $\mathscr{U}$ then there is a model geometry on $\mathbb{C}^{n \mid 2^{n}}$ with $\mathscr{U}$ as an automorphism group. This geometry will be given by a reduction of the frame bundle to a $U(1) \times S U(n)$ subbundle, along with a flat connection on this subbundle, and can be written down explicitly as in Sect. IV.

The spinor space $\Lambda^{*, 0}$ has a decomposition into its chiral subspaces $\Lambda^{\text {even,0 }}$ and $\Lambda^{\text {odd, } 0} . \mathbb{C}^{*}$ acts as automorphisms on $u$ by the transformations

$$
Q^{\text {even }} \rightarrow z Q^{\text {even }}, \quad Q^{\text {odd }} \rightarrow \bar{z}^{-1} Q^{\text {odd }} .
$$


Thus there is a natural extension $\mathbb{C}^{*} \times \mathscr{U}$ of $\mathscr{U}$ with even part $\mathbb{C}^{*} \times U(1) \times S U(n)$. The $\mathbb{C}^{*}$ can be thought of as an internal symmetry group which generates complex chiral transformations with respect to the decomposition of the spinor space.

In analogy with the definition of a superRiemannian structure bundle, we define a superKähler structure bundle as follows.

Definition 6. Let $X$ be a real supermanifold of dimension $2 n \mid 2^{n+1}$. Let $\mathscr{S}$ be a subspace of End $\left(\mathbb{R}^{2 n}, \mathbb{R}^{2^{n+1}}\right)$ which is $U(1) \times S U(n)$ invariant. A superKähler structure bundle $P$ is given by a reduction of the frame bundle of $X$ to the subgroup $(U(1) \times S U(n)) \tilde{\times} \mathscr{S}$ which is first-order flat. If $\mathscr{S}$ is $\mathbb{C}^{*} \times U(1) \times S U(n)$ invariant then a $\mathbb{C}^{*}$-extended superKähler structure bundle $P$ is given by a reduction of the frame bundle of $X$ to the subgroup $\left(\mathbb{C}^{*} \times U(1) \times S U(n)\right) \tilde{\times} \mathscr{S}$ which is first-order flat.

Given a superKähler structure bundle $P$, we can find local sections $\sigma$ of $P$ such that along $\sigma, T \in T_{0}+\operatorname{Im} \delta$.

Definition 7. A $\left(\mathbb{C}^{*}\right.$-extended) superKähler geometry is a reduction of $P$ to a $\left(\mathbb{C}^{*} \times\right) U(1) \times S U(n)$ subbundle $Q$ such that $T \in T_{0}+\operatorname{Im} \delta$ on $Q$.

Note in particular that a superKähler structure is automatically a superRiemannian structure, and that the reduced manifold is Kähler. As calculations for superKähler structures rapidly become very complicated, we will only discuss the cases of $n=1$ and $n=2$.

\section{A. One Complex Dimension}

Suppose that $X$ is a real supermanifold of dimension $2 / 4$ whose complexified cotangent bundle has a local coframe $\left\{\tau^{z}, \tau^{\bar{z}}, \tau^{\emptyset}, \tau^{\bar{\emptyset}}, \tau^{d z}, \tau^{d \bar{z}}\right\}$. Let us put

$$
\tau^{\theta_{1}}=\overline{\tau^{\emptyset}}, \quad \tau^{\theta_{2}}=\tau^{d z}
$$

Let us first consider a $\mathbb{C}^{*}$-extended superKähler geometry with $\mathscr{S}=\operatorname{End}\left(\mathbb{R}^{2}, \mathbb{R}^{4}\right)$. The representation $M: \mathbb{C} \oplus u(1) \rightarrow$ End $\left(\mathbb{C}^{1 / 2}\right)$ can be written in matrix form as $M_{A}{ }^{B}$ where the nonzero components are $M_{z}{ }^{z}, M_{\theta_{1}}^{{ }^{\theta_{1}}}$ and $M_{\theta_{2}}{ }^{{ }_{2}}$, and

$$
M_{\theta_{1}}^{\theta_{1}}+M_{\theta_{2}}^{\theta_{2}}=M_{z}{ }^{2}, \quad M_{z}{ }^{z}+M_{\bar{z}}^{\bar{z}}=0 \text {. }
$$

Let us assume that the torsion conditions are satisfied for a $\mathbb{C}^{*}$-extended superKähler geometry (we will not work out these conditions, although it is straightforward to do so). The torsion equations become

$$
\begin{aligned}
& d \tau^{z}+\tau^{z} \wedge \omega_{z}^{z}=-\tau^{\theta_{1}} \wedge \tau^{\theta_{2}} \\
& d \tau^{\theta_{1}}+\tau^{\theta_{1}} \wedge \omega_{\theta_{1}}^{\theta_{1}}=S_{11} \tau^{z} \wedge \tau^{\theta_{1}}+S_{12} \tau^{z} \wedge \tau^{\theta_{2}}+T_{11} \tau^{z} \\
& \wedge \tau^{\bar{\theta}_{1}}+T_{12} \tau^{z} \wedge \tau^{\bar{\theta}_{2}}+U_{1} \tau^{z} \wedge \tau^{\bar{z}}+V_{11} \tau^{\bar{z}} \\
& \wedge \tau^{\theta_{1}}+V_{12} \tau^{\bar{z}} \wedge \tau^{\theta_{2}}+W_{11} \tau^{\bar{z}} \wedge \tau^{\bar{\theta}_{1}} \\
& +W_{12} \tau^{\bar{z}} \wedge \tau^{\bar{\theta}_{2}}
\end{aligned}
$$




$$
\begin{aligned}
d \tau^{\theta_{2}}+\tau^{\theta_{2}} \wedge \omega_{\theta_{2}}^{\theta_{2}}= & S_{21} \tau^{z} \wedge \tau^{\theta_{1}}+S_{22} \tau^{z} \wedge \tau^{\theta_{2}}+T_{21} \tau^{z} \\
& \wedge \tau^{\bar{\theta}_{1}}+T_{22} \tau^{z} \wedge \tau^{\bar{\theta}_{2}}+U_{2} \tau^{z} \wedge \tau^{\bar{z}}+V_{21} \tau^{\bar{z}} \\
& \wedge \tau^{\theta_{1}}+V_{22} \tau^{\bar{z}} \wedge \tau^{\theta_{2}}+W_{21} \tau^{\bar{z}} \\
& \wedge \tau^{\bar{\theta}_{1}}+W_{22} \tau^{\bar{z}} \wedge \tau^{\bar{\theta}_{2}}
\end{aligned}
$$

We can use the freedom in $\operatorname{Im} \delta$ (for the structure group $\mathbb{C}^{*} \times \operatorname{Spin}(2)$ ) to set $S_{11}=S_{22}$ and $V_{11}=V_{22}$.

Proposition 26. $A \mathbb{C}^{*}$-extended superKähler geometry with $\mathscr{S}=\operatorname{End}\left(\mathbb{R}^{2}, \mathbb{R}^{4}\right)$ is equivalent to one with $\mathscr{S}=\operatorname{End}_{\mathbb{C}}\left(\mathbb{C}, \mathbb{C}^{2}\right)$. That is,

$$
V_{11}=V_{12}=V_{21}=V_{22}=W_{11}=W_{12}=W_{21}=W_{22}=0 \text {. }
$$

Proof. The exterior derivative of (357) gives

$$
\begin{aligned}
\tau^{z} \wedge \Omega_{z}{ }^{z}= & \tau^{\bar{z}} \wedge\left(V_{21} \tau^{\theta_{1}} \wedge \tau^{\theta_{1}}+V_{22} \tau^{\theta_{1}} \wedge \tau^{\theta_{2}}+W_{21} \tau^{\theta_{1}}\right. \\
& \wedge \tau^{\bar{\theta}_{1}}+W_{22} \tau^{\theta_{1}} \wedge \tau^{\bar{\theta}_{2}}+V_{11} \tau^{\theta_{1}} \wedge \tau^{\theta_{2}}+V_{12} \tau^{\theta_{2}} \\
& \left.\wedge \tau^{\theta_{2}}+W_{11} \tau^{\bar{\theta}_{1}} \wedge \tau^{\theta_{2}}+W_{12} \tau^{\bar{\theta}_{2}} \wedge \tau^{\theta_{2}}\right)\left(\bmod \tau^{z}\right) .
\end{aligned}
$$

The proposition follows.

It follows from Proposition 26 that we have the geometry considered in Sect. V.B.V. On the other hand, unextended superKähler geometries are less interesting, as the next proposition shows.

Proposition 27. An unextended super Kähler geometry has a flat connection, regardless of the U(1)-invariant subspace $\mathscr{S}$ of $\operatorname{End}\left(\mathbb{R}^{2}, \mathbb{R}^{4}\right)$.

Proof. Let us consider the most general possibility for $\mathscr{S}$, namely $\mathscr{S}=$ End $\left(\mathbb{R}^{2}, \mathbb{R}^{4}\right)$. We can consider an unextended superKähler geometry with connection to be an extended superKähler geometry with connection, whose curvature will then have to satisfy

$$
\Omega_{\theta_{1}}^{{ }^{\theta_{1}}}=\Omega_{\theta_{2}}^{{ }^{\theta_{2}}=1 / 2 \Omega_{z}{ }^{z}} .
$$

From Proposition 26, we can assume that $\mathscr{S}=\operatorname{End}_{\mathbb{C}}\left(\mathbb{C}, \mathbb{C}^{2}\right)$. From the results of Sect. V.B.V., we have that the relation $\Omega_{\theta_{1}}{ }^{\theta_{1}}=\Omega_{\theta_{2}}{ }^{\theta_{2}}$ implies

$$
\begin{aligned}
\left(\nabla_{\theta_{1}} U_{1}\right. & \left.+T_{11} \overline{T_{11}}+T_{12} \overline{T_{21}}\right) \tau^{z} \wedge \tau^{\bar{z}}+\nabla_{\theta_{1}} T_{11} \tau^{z} \wedge \tau^{\bar{\theta}_{1}} \\
& +\nabla_{\theta_{1}} T_{12} \tau^{z} \wedge \tau^{\bar{\theta}_{2}}-U_{1} \tau^{\theta_{2}} \wedge \tau^{\bar{z}}+T_{11} \tau^{\theta_{2}} \wedge \tau^{\bar{\theta}_{1}} \\
& +T_{12} \tau^{\theta_{2}} \wedge \tau^{\bar{\theta}_{2}}=\left(\nabla_{\theta_{2}} U_{2}+T_{22} \bar{T}_{22}+T_{21} \overline{T_{12}}\right) \tau^{z} \wedge \tau^{\bar{z}} \\
& +\nabla_{\theta_{2}} T_{22} \tau^{z} \wedge \tau^{\bar{\theta}_{2}}+\nabla_{\theta_{2}} T_{21} \tau^{z} \wedge \tau^{\bar{\theta}_{1}}-U_{2} \tau^{\theta_{1}} \wedge \tau^{\bar{z}} \\
& +T_{22} \tau^{\theta_{1}} \wedge \tau^{\bar{\theta}_{2}}+T_{21} \tau^{\theta_{1}} \wedge \tau^{\bar{\theta}_{1}}
\end{aligned}
$$

Thus

and so

$$
T_{11}=T_{12}=T_{21}=T_{22}=0,
$$

$$
U=0 \quad \text { and } \quad \Omega_{\theta_{1}}^{{ }^{\theta_{1}}}=\Omega_{\theta_{2}}{ }^{\theta_{2}}=\Omega_{z}{ }^{z}=0 \text {. }
$$




\section{B. Two Complex Dimensions}

Suppose that $X$ is a real supermanifold of dimension $4 \mid 8$ whose complexified cotangent bundle has a local coframe

$$
\left\{\tau^{z_{1}}, \tau^{z_{2}}, \tau^{\bar{z}_{1}}, \tau^{\bar{z}_{2}}, \tau^{\emptyset}, \tau^{d z_{1}}, \tau^{d z_{2}}, \tau^{d z_{1} \Lambda d z_{2}}, \tau^{\bar{\emptyset}}, \tau^{d \bar{z}_{1}}, \tau^{d \bar{z}_{2}}, \tau^{d \bar{z}_{1} \Lambda d \bar{z}_{2}}\right\}
$$

Let us put

$$
\tau^{\theta_{1}}=\tau^{\emptyset}, \quad \tau^{\theta_{2}}=\tau^{d z_{1}} . \quad \tau^{\theta_{3}}=\tau^{d z_{2}}, \quad \tau^{\theta_{4}}=\tau^{d z_{1} \Lambda d z_{2}} .
$$

Let us consider a $\mathbb{C}^{*}$-extended superKähler geometry with $\mathscr{S}=\operatorname{End}\left(\mathbb{R}^{4}, \mathbb{R}^{8}\right)$. The representation $M: \mathbb{C} \oplus u(1) \oplus s u(2) \rightarrow$ End $\left(\mathbb{C}^{2 \mid 4}\right)$ can be written in matrix form as $M_{A}{ }^{B}$ where the nonzero components are $\left\{M_{z_{\alpha}}^{{ }^{z_{\beta}}}, M_{\theta_{a}}^{\theta_{b}}\right\}$, and

$$
\begin{aligned}
& M_{\bar{z}_{\alpha}}^{\bar{z}_{\beta}}+M_{z_{\beta}}^{z_{\alpha}}=0, \quad M_{\theta_{5}}^{{ }^{\theta_{4}}}=M_{z_{2}}^{{ }^{z_{1}}}, \quad M_{\theta_{4}}^{{ }^{\theta_{5}}}=M_{z_{1}}^{z_{2}}, \\
& M_{\theta_{6}}{ }^{\theta_{6}}-M_{\theta_{3}}{ }^{\theta_{3}}=M_{z_{1}}{ }^{z_{1}}+M_{z_{2}}{ }^{z_{2}}, \quad M_{\theta_{4}}{ }^{\theta_{4}}-M_{\theta_{5}}{ }^{\theta_{5}}=M_{z_{1}}{ }^{z_{1}}-M_{z_{2}}{ }^{z_{2}}, \\
& M_{\theta_{4}}^{\theta_{4}}+M_{\theta_{5}}^{\theta_{5}}+\left(M_{\theta_{6}}^{\theta_{6}}+M_{\theta_{3}}^{{ }^{\theta_{3}}}\right)^{*}=0 \text {. }
\end{aligned}
$$

Let us assume that the torsion conditions are satisfied for a $\mathbb{C}^{*}$-extended superKähler geometry. The torsion equations become

$$
\begin{aligned}
& d \tau^{z_{1}}+\tau^{z_{1}} \wedge \omega_{z_{1}}^{z_{1}}+\tau^{z_{2}} \wedge \omega_{z_{2}}^{z_{1}} \\
& =-\left(\tau^{\bar{\theta}_{3}} \wedge \tau^{\theta_{4}}+\tau^{\bar{\theta}_{5}} \wedge \tau^{\theta_{6}}\right), \\
& d \tau^{z_{2}}+\tau^{z_{1}} \wedge \omega_{z_{1}}^{z_{2}}+\tau^{z_{2}} \wedge \omega_{z_{2}}^{z_{2}} \\
& =-\left(\tau^{\bar{\theta}_{3}} \wedge \tau^{\theta_{5}}-\tau^{\bar{\theta}_{4}} \wedge \tau^{\theta_{6}}\right), \\
& d \tau^{\theta_{3}}-\frac{1}{2} \tau^{\theta_{3}} \wedge\left(\omega_{z_{1}}{ }^{z_{1}}+\omega_{z_{2}}{ }^{z_{2}}-\bar{Y}\right) \\
& =\tau^{z_{1}} \wedge S_{1}{ }^{3}+\tau^{z_{2}} \wedge S_{2}{ }^{3}+\tau^{\bar{z}_{1}} \wedge S_{1}{ }^{3}+\tau^{\bar{z}_{2}} \wedge S_{\overline{2}}{ }^{3}, \\
& d \tau^{\theta_{4}}+\frac{1}{2} \tau^{\theta_{4}} \wedge\left(\omega_{z_{1}}{ }^{z_{1}}-\omega_{z_{2}}{ }^{z_{2}}-Y\right)+\tau^{\theta_{5}} \wedge \omega_{z_{2}}{ }^{z_{1}} \\
& =\tau^{z_{1}} \wedge S_{1}{ }^{4}+\tau^{z_{2}} \wedge S_{2}{ }^{4}+\tau^{\bar{z}_{1}} \wedge S_{\overline{1}}{ }^{4}+\tau^{\bar{z}_{2}} \wedge S_{\overline{2}}{ }^{4}, \\
& d \tau^{\theta_{5}}+\tau^{\theta_{4}} \wedge \omega_{z_{1}}{ }^{z_{2}}+\frac{1}{2} \tau^{\theta_{5}} \wedge\left(-\omega_{z_{1}}{ }^{z_{1}}+\omega_{z_{2}}{ }^{z_{2}}-Y\right) \\
& =\tau^{z_{1}} \wedge S_{1}{ }^{5}+\tau^{z_{2}} \wedge S_{2}{ }^{5}+\tau^{\bar{z}_{1}} \wedge S_{\overline{1}}{ }^{5}+\tau^{\bar{z}_{2}} \wedge S_{\overline{2}}{ }^{5}, \\
& d \tau^{\theta_{6}}+\frac{1}{2} \tau^{\theta_{6}} \wedge\left(\omega_{z_{1}}{ }^{z_{1}}+\omega_{z_{2}}{ }^{z_{2}}+\bar{Y}\right) \\
& =\tau^{z_{1}} \wedge S_{1}{ }^{6}+\tau^{z_{2}} \wedge S_{2}{ }^{6}+\tau^{\bar{z}_{1}} \wedge S_{1}{ }^{6}+\tau^{\bar{z}_{2}} \wedge S_{2}{ }^{6},
\end{aligned}
$$

where $Y$ represents the $\mathbb{C}^{*}$ part of the connection.

Proposition 28. With the above torsion conditions, the reduced Kähler manifold $M$ is locally Hermitian symmetric.

Proof. As the proof consists mostly of tedious calculations, we will only give a sketch. As a $\mathbb{C}^{*}$-extended superKähler geometry is also a superRiemannian geometry, we can use the results of Sect. V.D.II. In particular, the torsion equations for a $\mathbb{C}^{*}$-extended superKähler geometry are the same as those of a superRiemannian structure with $\omega_{\dot{\alpha}}^{\dot{\beta}}$ a diagonal matrix and $\omega_{i}{ }^{j}$ a diagonal matrix. Then $\Omega_{\dot{\alpha}}{ }^{\dot{\beta}}$ 
and $\Omega_{i}{ }^{j}$ will also be diagonal. The condition that $\Omega_{\dot{\alpha}}{ }^{\dot{\beta}}$ be diagonal gives

$$
W_{i}^{\dot{2}}=W_{i}^{i}=K=U_{11}^{\prime}=U_{2 i}^{\prime}=T^{\prime}=0 \text {. }
$$

The condition that $\Omega_{i}{ }^{j}$ be diagonal gives

$$
U=T_{11}=T_{22}=U^{\prime}=0
$$

Thus the possible nonzero superfields are $T_{12}=T_{21}, W_{i}^{i}=-W_{i}^{i}$ and $W^{\prime}$. To be slightly more general for a moment, let us consider the consequences of the Bianchi identities for the superRiemannian geometry of Sect. V.D.II, under the assumption $K=0$. One finds

$$
\begin{aligned}
& \nabla_{\phi} W_{\delta}^{\alpha}=0, \\
& \nabla_{k} \dot{\phi} W_{\alpha}^{\prime}=0 \text {, } \\
& \nabla_{l} \dot{v} W_{\dot{\omega}}^{\dot{\beta}}=\nabla_{l} \dot{\omega} W_{\dot{v}}^{\dot{\beta}}, \\
& \nabla_{\sigma}{ }^{k} W_{\alpha}^{\prime}{ }^{\varrho}=\nabla_{e^{k}} W_{\alpha}^{\prime \sigma}, \\
& \nabla_{k} \dot{\phi} U_{\beta \delta}^{\prime}=0 \text {, } \\
& \nabla_{k} \dot{\phi} T^{\prime i j}=0 \text {, } \\
& \nabla_{\phi} a T^{\prime(b c} \varepsilon^{d) a}=0, \\
& \nabla_{\phi}^{k} U^{\beta \gamma}=0 \text {, } \\
& \nabla_{\phi} k W_{\delta}^{\alpha}=0 \text {, } \\
& \nabla_{\phi^{k}} T_{i j}=0 \text {, } \\
& \nabla_{a} \dot{\phi} T_{(b c} \varepsilon_{d) a}=0 .
\end{aligned}
$$

We now use that $U=U^{\prime}=0$. If we use the fact that the only nonvanishing component of $T$ is $T_{12}$, (386) implies that $T_{12}$ is covariantly constant. Similarly, (378) implies that $W$ is covariantly constant. One finds from further Bianchi identities that $W^{\prime}$ must be covariantly constant. Then the $T^{* \text { even }} \wedge T^{* \text { even }}$ part of the curvature will be quadratic in the covariantly constant fields $T, W$ and $W^{\prime}$ and so will be covariantly constant. Thus the reduced space is a locally symmetric space.

Proposition 28 shows that the assumption of a first-order flat $\mathbb{C}^{*}$-extended superKähler structure is too strong to have interesting geometries. A similar situation is known to occur for $N>2$ extended supergravity theories in four dimensions [Ho2]. There it is found that it is necessary to allow a torsion $T$ with respect to the structure group $(\operatorname{Spin}(3,1) \times K) \tilde{\times} \mathscr{S}$ which is different from the model space torsion $T_{0}$, in order to write the corresponding supergravity theory.

In analogy, let us allow for a torsion with respect to the structure group $\left(\mathbb{C}^{*}\right.$ $\times U(1) \times S U(n)) \tilde{\times} \mathscr{S}$ which is different from the model space torsion. One condition on the torsion is that we want the torsion of the reduced space to vanish. That is, letting $V$ denote $\mathbb{C}^{2^{n}}$ and $W$ denote $\mathbb{C}^{n} \mid V$, we will want to allow the torsion $T$ to lie in a subspace of $\operatorname{Hom}(W \wedge W, W)$ which has zero intersection with $\operatorname{Hom}\left(\mathbb{C}^{n} \wedge \mathbb{C}^{n}, \mathbb{C}^{n}\right)$. The following definition seems to be appropriate. 
Definition 8 . A weak superKähler geometry is given by the definitions 6 and 7 , where the subspace $\mathscr{S}$ is now a $U(1) \times S U(n)$ invariant subspace of $\operatorname{End}(W, V)$.

(We do not consider $\mathbb{C}^{*}$-extended weak superKähler geometries because by writing out the torsion equations, one can see that a $\mathbb{C}^{*}$-extended weak superKähler geometry would be the same as an unextended weak superKähler geometry.)

We will look at weak superKähler geometries in one and two complex dimensions.

\section{One Complex Dimension}

Proposition 29. A weak super Kähler geometry in one complex dimension is equivalent to a $C^{*}$-extended superKähler geometry in one complex dimension.

Proof. The torsion equations for a weak superKähler geometry can be written in the form

$$
\begin{aligned}
d \tau^{z}+\tau^{z} \wedge \omega_{z}{ }^{z}=-\tau^{\theta_{1}} \wedge \tau^{\theta_{2}} & \\
d \tau^{\theta_{1}}+\tau^{\theta_{1}} \wedge \omega_{\theta_{1}}^{\theta_{1}}= & \tau^{z} \wedge A_{1}+\tau^{\bar{z}} \wedge B_{1}+\tau^{\theta_{1}} \wedge T_{1}{ }^{1}+\tau^{\theta_{2}} \\
& \wedge T_{2}{ }^{1}+\tau^{\overline{\theta_{1}}} \wedge T_{\overline{1}}{ }^{1}+\tau^{\overline{\theta_{2}}} \wedge T_{\overline{2}}{ }^{1} \\
d \tau^{\theta_{2}}+\tau^{\theta_{2}} \wedge \omega_{\theta_{2}}^{\theta_{2}}= & \tau^{z} \wedge A_{2}+\tau^{\bar{z}} \wedge B_{2}+\tau^{\theta_{1}} \wedge T_{1}{ }^{2}+\tau^{\theta_{2}} \\
& \wedge T_{2}{ }^{2}+\tau^{\overline{\theta_{1}}} \wedge T_{\overline{1}}{ }^{2}+\tau^{\overline{\theta_{2}}} \wedge T_{\overline{2}}{ }^{2}
\end{aligned}
$$

where $\omega_{z}{ }^{z}$ is imaginary and

$$
\omega_{\theta_{1}}^{\theta_{1}}=\omega_{\theta_{2}}^{\theta_{2}}=\frac{1}{2} \omega_{z}{ }^{z}
$$

Without loss of generality we may assume that each $T$ term has no $\tau^{z}$ or $\tau^{\bar{z}}$ component. Modulo $\tau^{z}$ and $\tau^{\bar{z}}$, the exterior derivative of (387) gives

$$
\begin{aligned}
0= & -\tau^{\theta_{1}} \wedge\left(\tau^{\theta_{1}} \wedge T_{1}^{2}+\tau^{\theta_{2}} \wedge T_{2}^{2}+\tau^{\overline{\theta_{1}}} \wedge T_{\overline{1}}^{2}+\tau^{\overline{\theta_{2}}} \wedge T_{\overline{2}}^{2}\right) \\
& +\left(\tau^{\theta_{1}} \wedge T_{1}^{1}+\tau^{\theta_{2}} \wedge T_{2}^{1}+\tau^{\overline{\theta_{1}}} \wedge T_{\overline{1}}^{1}+\tau^{\overline{\theta_{2}}} \wedge T_{\overline{2}}^{-1}\right) \wedge \tau^{\theta_{2}} .
\end{aligned}
$$

As the $T$ 's have no $\tau^{z}$ or $\tau^{\bar{z}}$ components, it follows that (391) is also true without the congruence condition. Taking (391) modulo $\tau^{\theta_{2}}$ gives that $\tau^{\theta_{1}} \wedge T_{1}^{2}+\tau^{\overline{\theta_{1}}} \wedge T_{\overline{1}}^{2}$ $+\tau^{\overline{\theta_{2}}} \wedge T_{\overline{2}}^{2}$ is proportionate to $\tau^{\theta_{2}}$. Similarly, $\tau^{\theta_{2}} \wedge T_{2}{ }^{1}+\tau^{\overline{\theta_{1}}} \wedge T_{\overline{1}}^{1}+\tau^{\overline{\theta_{2}}} \wedge T_{\overline{2}}{ }^{1}$ is proportionate to $\tau^{\theta_{1}}$. Thus we can write the torsion equations in the form

$$
\begin{aligned}
& d \tau^{z}+\tau^{z} \wedge \omega_{z}{ }^{z}=-\tau^{\theta_{1}} \wedge \tau^{\theta_{2}} \\
& d \tau^{\theta_{1}}+\tau^{\theta_{1}} \wedge \omega_{\theta_{1}}{ }^{\theta_{1}}=\tau^{z} \wedge A_{1}+\tau^{\bar{z}} \wedge B_{1}+\tau^{\theta_{1}} \wedge C_{1}, \\
& d \tau^{\theta_{2}}+\tau^{\theta_{2}} \wedge \omega_{\theta_{2}}{ }^{\theta_{2}}=\tau^{z} \wedge A_{2}+\tau^{\bar{z}} \wedge B_{2}+\tau^{\theta_{2}} \wedge C_{2} .
\end{aligned}
$$

Equation (391) becomes

$$
0=-\tau^{\theta_{1}} \wedge \tau^{\theta_{2}} \wedge\left(C_{1}+C_{2}\right)
$$


Thus $C_{1}+C_{2}=0$. We can now change the connection so that the torsion equations become

$$
\begin{aligned}
& d \tau^{z}+\tau^{z} \wedge \omega_{z}^{z}=-\tau^{\theta_{1}} \wedge \tau^{\theta_{2}},
\end{aligned}
$$

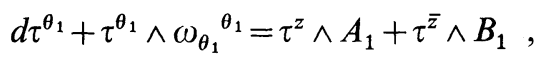

$$
\begin{aligned}
& d \tau^{\theta_{2}}+\tau^{\theta_{2}} \wedge{\omega_{\theta_{2}}}^{\theta_{2}}=\tau^{z} \wedge A_{2}+\tau^{\bar{z}} \wedge B_{2},
\end{aligned}
$$

where $\omega_{\theta_{1}}^{\theta_{1}}+\omega_{\theta_{2}}^{\theta_{2}}=\omega_{z}{ }^{z}$. These are exactly the torsion equations for a $C^{*}$-extended superKähler geometry.

\section{Two Complex Dimensions}

Proposition 30. A weak superKähler geometry in two complex dimensions is equivalent to a super Riemannian geometry in the sense of Sect. V.D.II in which $\omega_{\dot{\alpha}}^{\dot{\beta}}$ is a diagonal matrix.

Proof. The torsion equations for a weak superKähler geometry in two complex dimensions are

$$
\begin{aligned}
& d \tau^{z_{1}}+\tau^{z_{1}} \wedge \omega_{z_{1}}^{z_{1}}+\tau^{z_{2}} \wedge \omega_{z_{2}}^{z_{1}} \\
& =-\left(\tau^{\bar{\theta}_{3}} \wedge \tau^{\theta_{4}}+\tau^{\bar{\theta}_{5}} \wedge \tau^{\theta_{6}}\right), \\
& d \tau^{z_{2}}+\tau^{z_{1}} \wedge \omega_{z_{1}}^{z_{2}}+\tau^{z_{2}} \wedge \omega_{z_{2}}{ }^{z_{2}} \\
& =-\left(\tau^{\bar{\theta}_{3}} \wedge \tau^{\theta_{5}}-\tau^{\bar{\theta}_{4}} \wedge \tau^{\theta_{6}}\right), \\
& d \tau^{\theta_{3}}-\frac{1}{2} \tau^{\theta_{3}} \wedge\left(\omega_{z_{1}}^{{ }^{z_{1}}}+\omega_{z_{2}}{ }^{z_{2}}\right) \\
& =\tau^{z_{1}} \wedge S_{1}{ }^{3}+\tau^{z_{2}} \wedge S_{2}{ }^{3}+\tau^{\bar{z}_{1}} \wedge S_{\overline{1}}{ }^{3}+\tau^{\bar{z}_{2}} \wedge S_{\overline{2}}{ }^{3} \\
& +\tau^{\theta_{3}} \wedge T_{3}^{3}+\tau^{\theta_{4}} \wedge T_{4}{ }^{3}+\tau^{\theta_{5}} \wedge T_{5}^{3}+\tau^{\theta_{6}} \wedge T_{6}{ }^{3} \\
& +\tau^{\overline{\theta_{3}}} \wedge T_{\overline{3}}{ }^{3}+\tau^{\overline{\theta_{4}}} \wedge T_{\overline{4}}{ }^{3}+\tau^{\overline{\theta_{5}}} \wedge T_{\overline{5}}{ }^{3}+\tau^{\overline{\theta_{6}}} \wedge T_{\overline{6}}{ }^{3}, \\
& d \tau^{\theta_{4}}+\frac{1}{2} \tau^{\theta_{4}} \wedge\left(\omega_{z_{1}}{ }^{z_{1}}-{\omega_{z_{2}}}^{z_{2}}\right)+\tau^{\theta_{5}} \wedge \omega_{z_{2}}{ }^{z_{1}} \\
& =\tau^{z_{1}} \wedge S_{1}{ }^{4}+\tau^{z_{2}} \wedge S_{2}{ }^{4}+\tau^{\bar{z}_{1}} \wedge S_{\overline{1}}^{4}+\tau^{\bar{z}_{2}} \wedge S_{2}{ }^{4} \\
& +\tau^{\theta_{3}} \wedge T_{3}^{4}+\tau^{\theta_{4}} \wedge T_{4}^{4}+\tau^{\theta_{5}} \wedge T_{5}^{4}+\tau^{\theta_{6}} \wedge T_{6}^{4} \\
& +\tau^{\overline{\theta_{3}}} \wedge T_{\overline{3}}^{4}+\tau^{\overline{\theta_{4}}} \wedge T_{\overline{4}}^{4}+\tau^{\overline{\theta_{5}}} \wedge T_{\overline{5}}^{4}+\tau^{\overline{\theta_{6}}} \wedge T_{\overline{6}}^{4}, \\
& d \tau^{\theta_{5}}+\tau^{\theta_{4}} \wedge \omega_{z_{1}}{ }^{z_{2}}+\frac{1}{2} \tau^{\theta_{5}} \wedge\left(-\omega_{z_{1}}{ }^{z_{2}}+\omega_{z_{2}}{ }^{z_{2}}\right) \\
& =\tau^{z_{1}} \wedge S_{1}{ }^{5}+\tau^{z_{2}} \wedge S_{2}{ }^{5}+\tau^{\bar{z}_{1}} \wedge S_{\overline{1}}{ }^{5}+\tau^{\bar{z}_{2}} \wedge S_{\overline{2}}{ }^{5} \\
& +\tau^{\theta_{3}} \wedge T_{3}{ }^{5}+\tau^{\theta_{4}} \wedge T_{4}{ }^{5}+\tau^{\theta_{5}} \wedge T_{5}{ }^{5}+\tau^{\theta_{6}} \wedge T_{6}{ }^{5} \\
& +\tau^{\overline{\theta_{3}}} \wedge T_{\overline{3}}^{5}+\tau^{\overline{\theta_{4}}} \wedge T_{\overline{4}}^{5}+\tau^{\overline{\theta_{5}}} \wedge T_{\overline{5}}^{5}+\tau^{\overline{\theta_{6}}} \wedge T_{\overline{6}}{ }^{5}
\end{aligned}
$$




$$
\begin{aligned}
d \tau^{\theta_{6}}+ & \frac{1}{2} \tau^{\theta_{6}} \wedge\left({\omega_{z_{1}}}^{z_{1}}+\omega_{z_{2}}{ }^{z_{2}}\right) \\
= & \tau^{z_{1}} \wedge S_{1}{ }^{6}+\tau^{z_{2}} \wedge S_{2}{ }^{6}+\tau^{\bar{z}_{1}} \wedge S_{\overline{1}^{6}}{ }^{6}+\tau^{\bar{z}_{2}} \wedge S_{\overline{2}}{ }^{6}+\tau^{\theta_{3}} \wedge T_{3}{ }^{6} \\
& +\tau^{\theta_{4}} \wedge T_{4}{ }^{6}+\tau^{\theta_{5}} \wedge T_{5}{ }^{6}+\tau^{\theta_{6}} \wedge T_{6}{ }^{6}+\tau^{\overline{\theta_{3}}} \wedge T_{\overline{3}}{ }^{6} \\
& +\tau^{\overline{\theta_{4}}} \wedge T_{\overline{4}}{ }^{6}+\tau^{\overline{\theta_{5}}} \wedge T_{\overline{5}}{ }^{6}+\tau^{\overline{\theta_{6}}} \wedge T_{\overline{6}}{ }^{6} .
\end{aligned}
$$

Without loss of generality, we may assume that each $T$ term has no $\tau^{z_{1}}, \tau^{z_{2}}, \tau^{\bar{z}_{1}}$ or $\tau^{\bar{z}_{2}}$ component. Taking the exterior derivatives of (399) and (400) and using congruences shows that we may assume that the only nonzero entries of $T$ are $T_{3}^{3}, T_{\overline{6}}{ }^{3}, T_{4}^{4}$, $T_{\overline{5}}{ }^{4}, T_{5}{ }^{5}, T_{\overline{4}}{ }^{5}, T_{6}{ }^{6}$, and $T_{\overline{3}}{ }^{6}$, where we no longer assume that $T_{a b}{ }^{c}=T_{b a}{ }^{c}$. The exterior derivatives of (399) and (400) then give

$$
\begin{aligned}
0= & \tau^{\bar{\theta}_{3}} \wedge \tau^{\theta_{4}} \wedge\left(T_{4}^{4}+\overline{T_{3}^{3}}\right)+\tau^{\bar{\theta}_{3}} \wedge \tau^{\overline{\theta_{5}}} \wedge\left(T_{\overline{5}}^{4}+T_{\overline{3}}^{6}\right) \\
& +\tau^{\theta_{6}} \wedge \tau^{\theta_{4}} \wedge\left(\overline{T_{\overline{6}}{ }^{3}}+\overline{T_{\overline{4}}^{5}}\right)+\tau^{\theta_{6}} \wedge \tau^{\bar{\theta}_{5}} \wedge\left(T_{6}^{6}+\overline{T_{5}^{5}}\right)
\end{aligned}
$$

and

$$
\begin{aligned}
0= & \tau^{\bar{\theta}_{3}} \wedge \tau^{\theta_{5}} \wedge\left(T_{5}^{5}+\overline{T_{3}^{3}}\right)+\tau^{\overline{\theta_{3}}} \wedge \tau^{\overline{\theta_{4}}} \wedge\left(T_{\overline{4}}^{5}-T_{\overline{3}^{6}}\right) \\
& +\tau^{\theta_{6}} \wedge \tau^{\theta_{5}} \wedge\left(\overline{{T_{\overline{6}}}^{3}}-\overline{T_{\overline{5}}^{4}}\right)+\tau^{\overline{\theta_{4}}} \wedge \tau^{\theta_{6}} \wedge\left(-T_{6}^{6}-\overline{T_{4}^{4}}\right) .
\end{aligned}
$$

From (405) it follows that there are functions $V, W, X$, and $Y$ such that

$$
\begin{aligned}
& T_{4}^{4}+\overline{T_{3}^{3}}=\tau^{\bar{\theta}_{5}} X+\tau^{\theta_{6}} Y, \\
& T_{\overline{5}^{4}}+{T_{\overline{3}}}^{6}=-\tau^{\theta_{4}} X+\tau^{\theta_{6}} W \\
& \overline{T_{\overline{6}}^{3}}+\overline{T_{\overline{4}}^{5}}=-\tau^{\bar{\theta}_{3}} Y+\tau^{\bar{\theta}_{5}} \mathrm{~V}, \\
& T_{6}^{6}+\overline{T_{5}^{5}}=-\tau^{\bar{\theta}_{3}} W-\tau^{\theta_{4}} \mathrm{~V} .
\end{aligned}
$$

Similarly, it follows from (406) that there are functions $A, B, C$ and $D$ such that

$$
\begin{aligned}
& T_{5}^{5}+\overline{T_{3}^{3}}=\tau^{\overline{\theta_{4}}} A+\tau^{\theta_{6}} B, \\
& T_{\overline{4}}^{5}-T_{\overline{3}^{6}}=-\tau^{\theta_{5}} A+\tau^{\theta_{6}} C \\
& \overline{T_{\overline{6}}^{3}}-\overline{T_{\overline{5}}^{4}}=-\tau^{\bar{\theta}_{3}} B+\tau^{\bar{\theta}_{4}} D \\
& T_{6}^{6}+\overline{T_{4}^{4}}=\tau^{\bar{\theta}_{3}} C+\tau^{\theta_{5}} D .
\end{aligned}
$$

It follows from (407) and (411) that

$$
T_{4}^{4}-T_{5}^{5}=\tau^{\overline{\theta_{5}}} X+\tau^{\theta_{6}} Y-\tau^{\overline{\theta_{4}}} A-\tau^{\theta_{6}} B,
$$

and from (410) and (414) that

$$
\overline{T_{5}^{5}}-\overline{T_{4}^{4}}=-\tau^{\bar{\theta}_{3}} W-\tau^{\theta_{4}} V-\tau^{\bar{\theta}_{3}} C-\tau^{\theta_{5}} D .
$$


Then from (415) and (416) we have that $X-\bar{D}=0, Y-B=0, A+\bar{V}=0$ and $W+C=0$. If we put

$$
\begin{aligned}
& T^{\prime}{ }_{4}^{4}=T_{4}{ }^{4}-\tau^{\bar{\theta}_{5}} X, \\
& T^{\prime}{ }^{3}=T_{3}{ }^{3}-\tau^{\overline{\theta_{6}}} \bar{Y} \text {, } \\
& T^{\prime}{ }^{4}=T_{5}^{4}+\tau^{\theta_{4}} X \text {, } \\
& T^{\prime}{ }^{6}=T_{\overline{3}}^{6}-\tau^{\theta_{6}} W \text {, } \\
& T^{\prime}{ }^{3}=T_{\overline{6}}{ }^{3}+\tau^{\theta_{3}} \bar{Y} \text {, } \\
& T^{\prime}{ }_{4}^{5}=T_{4}{ }^{5}-\tau^{\theta_{5}} \bar{V}, \\
& T^{\prime}{ }_{6}^{6}=T_{6}{ }^{6}+\tau^{\bar{\theta}_{3}} W \text {, } \\
& T^{\prime}{ }_{5}^{5}=T_{5}{ }^{5}+\tau^{\overline{\theta_{4}}} \bar{V} \text {, }
\end{aligned}
$$

then we have

$$
\overline{T^{\prime}{ }_{3}^{3}}=-T_{4}^{\prime 4}=-T^{\prime}{ }_{5}^{5}=\overline{T_{6}^{\prime 6}}
$$

and

$$
T^{\prime}{ }^{4}=-T^{\prime} \overline{3}^{6}=-T_{4}^{\prime}{ }^{5}=T^{\prime} \overline{6}^{3}
$$

One can check that replacing $T$ by $T^{\prime}$ does not change the torsion Eqs. (399)-(404). After moving the $T^{\prime}$ terms to the left-hand side of the torsion equations, on can check that the torsion equations become equivalent to those of Sect. V.D.II, provided that $\omega_{\dot{\alpha}}^{\dot{\beta}}$ is diagonal matrix.

We have not analyzed the consequences of the Bianchi identities for a weak superKähler geometry in two complex dimensions. However, the following seems reasonable:

Conjecture. There are solutions of the Bianchi identities for a weak superKähler geometry in two complex dimensions which give a non-covariantly-constantcurvature reduced space.

\section{Gauge Theory}

In order to give a space of superconnections suitable for gauge theory, it is necessary to put some constraints on the curvature of a connection. From the discussion of Sect. IV, we know that if $X$ is a supermanifold with a reduction of the frame bundle to the group $G=\left(\operatorname{Spin}\left(p_{+}, p_{-}\right) \times K\right) \tilde{\times} \mathscr{S}$ then there is a well defined odd subbundle $T_{\text {odd }} X$ of $T X$ with an action of $\operatorname{Spin}\left(p_{+}, p_{-}\right) \times K$ via the representation $\tilde{\varrho}$. Let $H$ be an ordinary Lie group and let $A$ be an $H$-connection on a vector bundle over $X$. The curvature constraints can be summarized by saying that the curvature $F$ of $A$ vanishes on some subspace $V$ of $T_{\text {odd }} X \wedge T_{\text {odd }} X$ which is $\operatorname{Spin}\left(p_{+}, p_{-}\right) \times K$ invariant. We must admit that we do not have any general way to determine the appropriate $V$, a maximal subspace such that $F$ is not implied to vanish identically. (For example, for $N=4$ gauge theory in four dimensions, there is a self-duality condition on the curvature [So], which does not occur for $N<4$. This condition 
exists because the internal symmetry group of the superconformal group for $N=4$ is $S U(4)$ rather than $U(4)$.) The curvature constraints for extended supergauge theory in four-dimensional Minkowski space can be found in [So]. Let us note that it is often the case that there is a subspace $W$ of $T_{\text {odd }} X$ (or $T_{\text {odd }} X \otimes \mathbb{C}$ ) such that $W \wedge W \subset V\left(\right.$ or $V \otimes \mathbb{C}$ ). Then $\left.A\right|_{W}$ can be locally written in the form $\left.g^{-1} d g\right|_{W}$, where $g$ takes value in $H$ (or $H_{\mathbb{C}}$ ). The field $g$ is the prepotential in physics [GGRS]. Let us also note that it seems to be necessary to have at least a superconformal structure on $X$, i.e. a reduction of the frame bundle of $X$ to $\left(\mathbb{R}^{+} \times \operatorname{Spin}\left(p_{+}, p_{-}\right) \times K\right) \tilde{\times} \mathscr{S}$, in order to define the space of connections for a supergauge theory.

As an example, let $H$ be a Lie group and consider a gauge theory on Euclidean $\mathbb{R}^{4 / 8}$ with structure group $H$. The Yang-Mills action was given in components in $[\mathrm{Zu}]$ as

$$
\begin{aligned}
L= & \int_{\mathbb{R}^{4}}\left[\frac{1}{2}|F(A)|^{2}-\frac{1}{2}\left|D_{A} B\right|^{2}+\frac{1}{2}\left|D_{A} C\right|^{2}\right. \\
& +\frac{1}{2} i\left(\left\langle\psi, \gamma^{\mu}\left(D_{A}\right)_{\mu} \psi\right\rangle+\left\langle\gamma^{\mu}\left(D_{A}\right)_{\mu} \psi, \psi\right\rangle\right) \\
& \left.-i\left\langle\psi,\left[B-\gamma_{5} C, \psi\right]\right\rangle-\frac{1}{2}|[B, C]|^{2}\right] .
\end{aligned}
$$

Here $A$ is an $H$-connection on $\mathbb{R}^{4}, B$ and $C$ are cross-sections of the ad $(H)$ bundle, $\psi$ is a cross-section of the $S \otimes \operatorname{ad}(H)$ bundle (where $S$ denotes the complex spinor bundle) and $\gamma_{5}$ is the Hermitian chirality matrix.

In order to write the superspace version, let us note that the representation of Spin (4) on $\mathbb{R}^{8}$ is quaternionic. Let $\mathbb{R}^{4 / 8}=\mathbb{H}^{1 / 2}$ have quaternionic coordinates $x, \theta$ and $\theta^{\prime}$. Let $Q$ denote $d \theta, Q^{\prime}$ denote $d \theta^{\prime}$ and $P$ denote $d x+\frac{1}{2} \theta d \theta^{\prime}-\frac{1}{2} d \theta \theta^{\prime}$. Let $A$ be an $H$-connection on $\mathbb{R}^{4 / 8}$ and let $F$ denote its curvature. The right constraints for $F$ turn out to be

$$
F=\operatorname{Re}\left(W^{\prime} \bar{Q} \wedge Q+\overline{W Q^{\prime}} \wedge Q^{\prime}\right)(\bmod P)
$$

for some $h$-valued superfunctions $W$ and $W^{\prime}$. Let $D_{Q}$ denote the $\mathbb{H}$-valued differential operator

$$
D_{Q}=D_{\theta^{0}}+i D_{\theta^{1}}+j D_{\theta^{2}}+k D_{\theta^{3}},
$$

and similarly for $D_{Q^{\prime}}$. The Bianchi identities imply that

$$
D_{Q} W^{\prime}=D_{Q^{\prime}} \bar{W}=0 \text { and } \overline{D_{Q}} D_{Q} W-D_{Q^{\prime}} \overline{D_{Q^{\prime}}} W^{\prime}=0
$$

and that $F$ can be completely expressed in terms of $W$ and $W^{\prime}$ by

$$
\begin{aligned}
& F=\operatorname{Re}\left(W^{\prime} \bar{Q} \wedge Q+W \bar{Q}^{\prime} \wedge Q^{\prime}-2 Q D_{Q^{\prime}} W^{\prime} \wedge \bar{P}\right. \\
& -2 \bar{Q}^{\prime} \overline{D_{Q}} W \wedge P-\frac{1}{4} \overline{D_{Q^{\prime}}} D_{Q^{\prime}} W^{\prime} \bar{P} \wedge P \\
& \left.-\frac{1}{4} D_{Q} \overline{D_{Q}} W P \wedge \bar{P}\right) \text {. }
\end{aligned}
$$

The Yang-Mills equations become

$$
\overline{D_{Q}} D_{Q} W=D_{Q^{\prime}} \overline{D_{Q^{\prime}}} W^{\prime}=0 \text {. }
$$

Self-dual solutions correspond to $W^{\prime}=0$ and anti-self-dual solutions correspond to $W=0$. One could also work out the superspace Yang-Mills equations for a gauge theory on a general superRiemannian manifold of dimension $4 \mid 8$, the geometry of which was given in Sect. V.D.II. 


\section{Supergeometries as Submanifolds}

We will briefly sketch the relationship between the present paper and the work of [OS, RS, Ma]. We have seen that an important feature of a superRiemannian or superconformal geometry is the existence of an well-defined odd subspace of the tangent space. One way to construct supermanifolds with such subspaces is the following. Suppose that $X$ is a real submanifold of a complex (or quaternionic) affine superspace $Y$. Then the tangent space to $X$ will inherit a subspace which is invariant under the complex (or quaternionic) structure. If this subspace is an odd subspace of maximal dimension then one has a candidate for a superconformal structure on $X$. (One also needs a nondegeneracy condition on the commutator of the subspace.) Conversely, given an odd subspace with a complex (or quaternionic) structure on a general superconformal manifold $X$, the appropriate integrability conditions will give necessary conditions for $X$ to be locally realizable as a submanifold of an affine space. The torsion equations for a superconformal structure can be interpreted as the integrability conditions for the complex (or quaternionic) subspace of the tangent space, in analogy to what happens for CR manifolds. This was shown for $N=1$ supergravity in Minkowski 4-space in [RS]. (A superRiemannian geometry can be considered to be the analogue of the pseudoHermitian geometry of Webster [We].)

When we have such an extrinsic description of conformal supergeometry, one can ask which submanifold corresponds to the model geometry. It turns out that the model submanifold is given locally as the zero set of a set of real quadratic equations in the complex (or quaternionic) affine superspace. Thus the superconformal curvature tensor measures whether a given submanifold $X$ is locally equivalent to the model submanifold with respect to complex-analytic (or quaternionic-analytic) maps of the affine superspace.

One finds such a description of the model supermanifold when it is given as the subspace of a complex (or quaternionic) Grassmannian supermanifold on which a quadratic form vanishes. We find in particular the following cases.

\section{I. $(2,2)$ Supersymmetry in Minkowski 2-Space}

The model space is

$$
\begin{aligned}
X= & \left\{\mathbb{C}^{0 \mid 1} \text { planes } P \text { in } \mathbb{C}^{1 \mid 2}: \text { the quadratic form } \bar{x} x+\overline{\eta_{1}} \eta_{2}-\overline{\eta_{2}} \eta_{1}\right. \\
& \text { on } \left.\mathbb{C}^{1 / 2} \text { vanishes on } P\right\} \times\left\{\mathbb{C}^{0 \mid 1} \text { planes } Q \text { in } \mathbb{C}^{1 / 2}:\right. \\
& \text { the quadratic form } \left.\bar{x} x+\overline{\eta_{1}} \eta_{2}-\overline{\eta_{2}} \eta_{1} \text { on } \mathbb{C}^{1 / 2} \text { vanishes on } Q\right\} .
\end{aligned}
$$

If we parametrize a subspace of $X$ by

$$
P=\operatorname{span}((c, 1, a)), \quad Q=\operatorname{span}((d, 1, b))
$$

then $X$ is locally described as

$$
\left\{(a, c, b, d) \in \mathbb{C}^{1 \mid 1} \times \mathbb{C}^{1 \mid 1}: a-\bar{a}=-\bar{c} c, b-\bar{b}=-\bar{d} d\right\} .
$$


II. $N=2$ Supersymmetry in Euclidean 3-Space

The model space is

$$
\begin{aligned}
& X=\left\{\mathbb{H}^{1 \mid 0} \text { planes } P \text { in } \mathbb{H}^{2 / 2}\right. \text { : the quadratic form } \\
& \overline{x_{1}} x_{2}+\overline{x_{2}} x_{1}+\overline{\eta_{1}} \eta_{2}-\overline{\eta_{2}} \eta_{1} \text { on } \mathrm{H}^{2 / 2} \\
& \text { vanishes on } P\} \text {. }
\end{aligned}
$$

If we parametrize a subspace of $X$ by

$$
P=\operatorname{span}((1, a, b, c)),
$$

then $X$ is locally described as

$$
\left\{(a, b, c) \in \mathbb{H}^{1 / 2}: a+\bar{a}+\bar{b} c-\bar{c} b=0\right\} .
$$

III. $N=1$ Supersymmetry in Minkowski 4-Space

The model space is

$$
\begin{aligned}
X= & \left\{\mathbb{C}^{0 / 2} \text { planes } P \text { in } \mathbb{C}^{1 / 4}:\right. \text { the quadratic form } \\
& \bar{x} x+\bar{\eta}_{0} \eta_{2}-\bar{\eta}_{2} \eta_{0}+\bar{\eta}_{1} \eta_{3}-\bar{\eta}_{3} \eta_{1} \text { on } \mathbb{C}^{1 / 4} \\
& \text { vanishes on } P\} .
\end{aligned}
$$

If we parametrize a subspace of $X$ by

$$
P=\operatorname{span}((x, a, b, 1,0),(y, c, d, 0,1)),
$$

then $X$ is locally described as

$$
\left\{(a, b, c, d, x, y) \in \mathbb{C}^{4 \mid 2}: \bar{a}-a=\bar{x} x,-c+\bar{b}=\bar{x} y, \bar{d}-d=\bar{y} y\right\} .
$$

IV. $N=1$ Supersymmetry in Minkowski 6-Space

The model space is

$$
\begin{aligned}
X= & \left\{\mathrm{H}^{0 / 2} \text { planes } P \text { in } \mathbb{H}^{1 / 4}:\right. \text { the quadratic form } \\
& \bar{x} x+\bar{\eta}_{0} \eta_{2}-\bar{\eta}_{2} \eta_{0}+\bar{\eta}_{1} \eta_{3}-\bar{\eta}_{3} \eta_{1} \text { on } \mathbb{H}^{1 / 4} \\
& \text { vanishes on } P\} .
\end{aligned}
$$

If we parametrize a subspace of $X$ by

$$
P=\operatorname{span}((x, a, b, 1,0),(y, c, d, 0,1)),
$$

then $X$ is locally described as

$$
\left\{(a, b, c, d, x, y) \in \mathbb{H}^{4 \mid 2}: \bar{a}-a=\bar{x} x,-c+\bar{b}=\bar{x} y, \bar{d}-d=\bar{y} y\right\} .
$$




\section{References}

[At] M. Atiyah: Circular symmetry and the stationary phase approximation. Astérisque 131, 43 (1985)

[Ba] M. Batchelor: The structure of supermanifolds. Trans. AMS 253, 329 (1979)

[CM] S. Chern, J. Moser: Real hypersurfaces in complex manifolds. Acta Math. 133, 219 (1975)

[CW] C. Crnkovic, E. Witten: Covariant description of canonical formalism in geometric theories. Newton Tercentenary Volume. Hawking, S., Israel, W. (eds.) Cambridge: Cambridge University Press 1987

[Dr] N. Dragon: Torsion and curvature in extended supergravity. Z. Phys. C2, 29 (1979)

[DS] J. Dell, L. Smolin: Graded manifold theory as the geometry of superspace. Commun. Math. Phys. 66, 197 (1979)

[Ei] L. Eisenhart: Riemannian geometry. Princeton, N.J.: Princeton University Press 1926

[Ga] K. Gawedzki: Supersymmetries - mathematics of supergeometry. Ann. Inst. H. Poincaré Sect. A27, 335 (1977)

[GGRS] S. Gates, Jr., M. Grisaru, M. Rocek, W. Siegel: Superspace or 1001 lessons in supersymmetry. Reading, MA: Benjamin Cummings 1983

[GN] S. Giddings, P. Nelson: The geometry of superRiemann surfaces. Commun. Math. Phys. 116, 607 (1988)

[Gu] V. Guillemin: The integrability problem for $G$-structures. Trans. Am. Math. Soc. 116, 544 (1965)

[Hi] N. Hitchin: Harmonic spinors. Adv. Math. 14, 1 (1974)

[Ho1] P. Howe: Super Weyl transformations in two dimensions. J. Phys. A12, 393 (1979)

[Ho2] P. Howe: Supergeometry in superspace. Nucl. Phys. B 199, 309 (1982)

[HP] P. Howe, G. Papadopoulos: $N=2, d=2$ supergeometry. Class. Quant. Grav. 4, 11 (1987)

[HST] P. Howe, G. Sierra, P. Townshend: Supersymmetry in six dimensions. Nucl. Phys. B221, 331 (1983)

[Ka] V. Kac: A sketch of Lie superalgebra theory. Commun. Math. Phys. 53, 31 (1977)

[Kob] S. Kobayashi: Transformation groups in differential geometry. Berlin Heidelberg New York: Springer 1972

[Kos] B. Kostant: Graded manifolds, graded Lie theory and prequantization. Lecture Notes in Mathematics, Vol. 570. Berlin Heidelberg New York: Springer 1977

[KN] S. Kobayashi, K. Nomizu: Foundations of differential geometry, Vol. 1. New York: Interscience 1963

[KS] J. Kijowski, W. Szczyrba: A canonical structure for classical field theories. Commun. Math. Phys. 46, 183 (1976)

[Le] D. Leites: Introduction to the theory of supermanifolds. Russ. Math. Surv. 35, 1 (1980)

[Lo] J. Lott: Supersymmetric path integrals. Commun. Math. Phys. 108, 605 (1987)

[LR] C. Lebrun, M. Rothstein: Moduli of superRiemann surfaces. Commun. Math. Phys. 117, 159 (1988)

[Ma] Y. Manin: Gauge field theory and complex geometry. Berlin Heidelberg New York: Springer 1988

[MN] G. Moore, P. Nelson: Heterotic geometry. Nucl. Phys. B274, 509 (1986)

[NS] A, Neveu, J. Schwarz: Factorizable dual model of pions. Nucl. Phys. B31, 86 (1971)

[OS] V. Ogievetsky, E. Sokatchev: The simplest group of Einstein supergravity. Yad. Fiz. 31, 264 (1980)

[Pa] R. Palais: Foundations of global nonlinear analysis. New York: Benjamin 1968

[RS] A. Rosly, A. Schwarz: Geometry of $N=1$ supergravity. Commun. Math. Phys. 95, 161 (1984)

[Se] I. Segal: Mathematical problems of relativistic physics. AMS Lectures in Applied Math., AMS 1963

[So] M. Sohnius: Bianchi identities for supersymmetric gauge theories. Nucl. Phys. B136, 461 (1978)

[Sp] D. Spencer: Deformation of structures on manifolds defined by transitive continuous pseudogroups. Ann. Math. 76, (2) 306 (1962) 
[St] S. Sternberg: Lectures on differential geometry. Englewood Cliffs, N.J.: Prentice-Hall 1964

[ST] G. Sierra, P. Townshend: The gauge-invariant $N=2$ supersymmetric $\sigma$-model with general scalar potential. Nucl. Phys. B233, 289 (1984)

[WB] J. Wess, J. Bagger: Supersymmetry and supergravity. Princeton, N.J.: Princeton University Press 1983

[We] S. Webster: Pseudo-Hermitian structures on a real hypersurface. J. Diff. Geom. 13, 25 (1978)

[WZ] J. Wess, B. Zumino: Superspace formulation of supergravity. Phys. Lett. B66, 361 (1977)

$[\mathrm{Zu}] \quad$ B. Zumino: Euclidean supersymmetry and the many-instanton problem. Phys. Lett. B69, 369 (1977)

[RS2] A. Rosly, A. Schwarz: Geometry of $N=1$ supergravity, II. Commun. Math. Phys. 96, $285(1984)$

Communicated by S.-T. Yau 
